\title{
PREPROCESSING AND POSTPROCESSING FOR MATERIALS BASED ON THE HOMOGENIZATION METHOD WITH ADAPTIVE FINITE ELEMENT METHODS
}

\author{
José Miranda GUEDES and Noboru KIKUCHI \\ Department of Mechanical Engineering and Applied Mechanics, The University of Michigan, \\ Ann Arbor, Michigan 48109, U.S.A.
}

Received 22 August 1989

\begin{abstract}
This paper discusses the homogenization method to determine the effective average elastic constants of linear elasticity of general composite materials by considering their microstructure. After giving a brief theory of the homogenization method, a finite element approximation is introduced with convergence study and corresponding error estimate. Applying these, computer programs PREMAT and POSTMAT are developed for preprocessing and postprocessing of material characterization of composite materials. Using these programs, the homogenized elastic constants for macroscopic stress analysis are obtained for typical composite materials to show their capability. Finally, the adaptive finite element method is introduced to improve the accuracy of the finite element approximation.
\end{abstract}

\section{Introduction}

The role of composite materials is becoming increasingly important in industry. Their microstructure character provides a good weight/strength ratio that makes them suitable for a large variety of applications ranging from sports goods to space aircraft and their application in high performance structures makes thermal/stress analysis a major concern. It is extremely difficult to analyze such structures, including each individual microstructure, due to the high degree of material heterogeneity. A natural way to overcome this difficulty is to find some kind of equivalent material model without needing to represent each individual microstructure. This model should characterize the average mechanical behavior as well as represent the effect of the composite material heterogeneities.

Much research has focused on defining equivalent mechanical properties of composite materials and on determining their dependence on the different components. This research activity provided several different methods of computing equivalent material properties. Although some of these methods are engineering based, they quite often show a good agreement with other empirical methods and/or available experimental data. A survey of such activity is given in [1] and references therein provide good insight into this approach.

A mathematical counterpart to such engineering methods appears in the 70's with the name of homogenization theory. Since then this theory has been the subject of a large amount of research in area of applied mathematics. The fundamentals of this theory can be found, among others, in [2-9]. A review of the recent progresses in the mathematical modelling of composite materials as well as an extensive reference list can be found in [10]. 
In homogenization theory it is usually assumed that the composite material is locally formed by the spatial repetition of very small microstructures, 'microscopic' cells, when compared with the overall 'macroscopic' dimensions of the structure of interest. In other words, it is assumed that the material properties are periodic functions of the microscopic variable, where the period is very small compared with the macroscopic variable. This assumption enables the computation of equivalent material properties by a limiting process when the microscopic cell size is reduced to zero.

The homogenization method is advantageous because it is a rigorous mathematical theory. It can also provide reasonabie solutions for some problems where the experimental data is not available, or for which only bounds for the equivalent material constants can be found by other theories (see for example [11, 12]).

Several cases of homogenization can be found in [7]. This work considers the cases of composite materials formed by elastic media with periodic holes and rigid inclusions, and fiber reinforced elastic materials where slipping without decohesion may occur between the fiber and the matrix with linear, nonlinear and viscous tangential forces at the slipping boundary. This last class of problems is particularly interesting due to the existence of nonlinearity effects. All these cases are mathematically formulated and discussed. Numerical procedures are also introduced and examples for two-dimensional cases are worked out.

Another set of numerical applications can be found in [13]. This work at INRIA considers the numerical implementation of the computation of equivalent material elastic constants, or homogenized coefficients, for two- and three-dimensional examples and presents several examples.

The purpose of the present work is to study the numerical accuracy of the finite element solutions for the equivalent material properties, their influence in the overall numerical solution and ways to improve this accuracy, as well as to study the applicability of the homogenization method for the stress analysis of composite materials. This work is formed by 5 sections.

In Section 2 a brief description of homogenization theory is given for the case of linear elastic materials. In this section the problem of finding the equivalent material properties of a composite material involving microscale holes subjected to tractions is presented. The use of homogenization technique introduces three uncoupled problems: two on the 'microscopic' level and one on the 'macroscopic' level. The 'microscopic' level problems enable the computation of the homogenized coefficients as well as a kind of 'residual stresses' that will be used to solve the 'macroscopic' problem.

In Section 3 the numerical solution of the before-mentioned problems is considered. The finite element method is introduced to compute the homogenized coefficients, the 'residual stresses' and the global displacements and stress fields. Also, a priori error estimations are derived for the finite element approximations of the above quantities.

In Section 4 the homogenization method is used to introduce the idea of material preprocessing (PREMAT), to compute homogenized material properties for composites, and material postprocessing (POSTMAT), to compute local distribution of stresses and strains within the microscopic level. These are then used to solve stress analyses problems of composite materials. It is noted that there are few similar examples to the ones studied in this work.

In Section 5 an adaptive finite element method is introduced in order to improve the 
accuracy of the numerical solution. This adaptive method is based on the a priori error estimations. Its implementation is described and examples are performed.

In the last section some general remarks are made.

\section{Review of the homogenization theory}

Consider a composite material formed by the spatial repetition of a base cell made of different materials as shown in Fig. 2.1. For the sake of simplicity oniy two-dimensional figures are presented, although the following is developed for the three-dimensional case. Assume that a body is made of, for example, two different materials whose mixture is represented by a base cell that is very small, of order $\varepsilon$ (where $\varepsilon$ is a very small positive number) compared with the dimensions of the structural body. If the body is subjected to some load and boundary conditions, the resulting deformation and stresses, in general, rapidly vary from point to point because of repetition of microscopic base cells producing heterogeneity. In other words, with the high level of heterogeneity within the material, these quantities also vary rapidly within a very small neighborhood $\varepsilon$ of a given point $x$. Thus it is reasonable to claim that all quantities have two explicit dependences. One is on the 'macroscopic' level $x$, and the other is on the 'microscopic' level $x / \varepsilon$, i.e., letting $g$ be a general function, $g=g(x, x / \varepsilon)$. Due to the periodic nature of the microstructure, the dependence of a function on the 'microscopic' variable $y=x / \varepsilon$ is also periodic.

To solve such a problem using finite element methods would be almost impossible, since discretization of the body becomes enormous in order to represent detailed structure of the microscopic material constitution. Thus it would be desirable to develop a method that can reflect the microscopic structure without looking at details of all of the material points of the body, whenever the mechanical behavior of the macroscopic body is in question. The homogenization method has been developed to realize this kind of approach. Furthermore, with this method, the mechanical behavior of microstructure can be approximately predicted by a postprocessing of the macroscopic stress analysis. The homogenization method is introduced in the remainder of this section.

Let $\Omega$ be an open subset of $\mathbb{R}^{3}$ with a smooth, say Lipschitz, boundary $\Gamma$ (see Fig. 2.2). Let

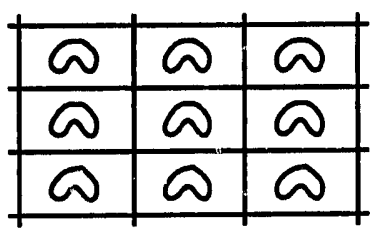

Fig. 2.1. Composite structure.

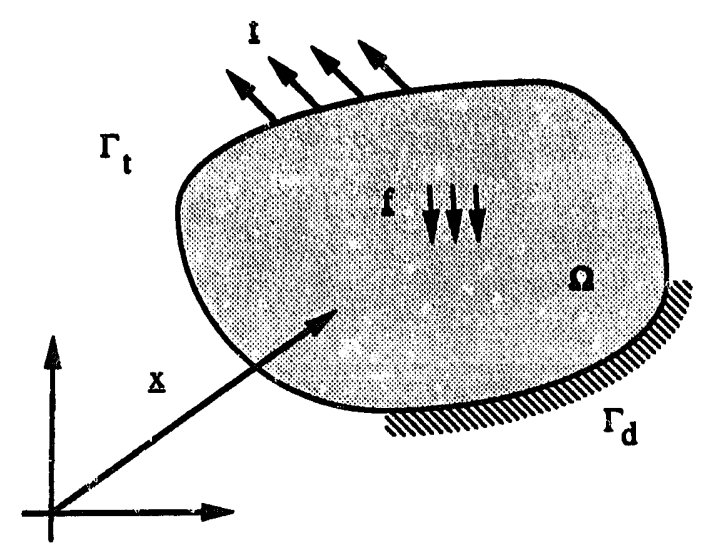

Fig. 2.2. General elasticity problem. 


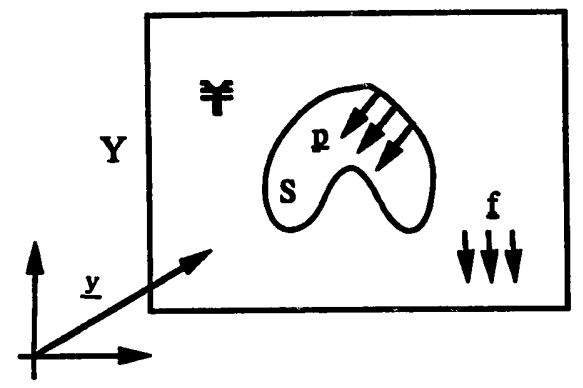

Fig. 2.3. Base cell of the composite.

$Y$ be an open rectangular parallelepiped in $\mathbb{R}^{3}$ (see Fig. 2.3) defined by

$$
Y=] 0, y_{1}^{0}[\times] 0, y_{2}^{0}[\times] 0, y_{3}^{0}[,
$$

let $\vartheta$ be an open subset of $Y$ with boundary

and let

$$
\partial \vartheta=S
$$

$$
\boldsymbol{Y}=\boldsymbol{Y} \backslash \overline{\boldsymbol{\vartheta}},
$$

where $Y$ is the solid part of the cell, $\bar{\vartheta}$ denotes the closure of $\vartheta$ and $Y$ represents the base cell of the composite microstructure. The material properties vary inside $Y$, and the set $\vartheta$ represents a hole inside $Y$. Define now

$$
\Theta(y)= \begin{cases}1 & \text { if } y \in Y, \\ 0 & \text { if } y \notin Y,\end{cases}
$$

and extend $\Theta$ to $\mathbb{P}^{3}$ by $\varepsilon$ periodicity, i.e., repeat the base cell in all three directions. Then define

$$
\Omega^{8}=\{x \in \Omega \mid \Theta(x / \varepsilon)=1\},
$$

i.e., $\boldsymbol{\Omega}^{8}$ is the solid part of the domain. Also define

$$
S^{\varepsilon}=\bigcup_{\alpha=1}^{\text {all cells }} S_{\alpha}
$$

and consider the following hypotheses:

1. $\Omega^{6}$ is a connected domain,

2. The hole(s) $\vartheta$ has sufficiently smooth boundary(ies) $S$,

3. None of the holes $S$ intersects the boundary $\Gamma$ of $\Omega$. 
Now let

$$
V^{\varepsilon}=\left\{v \in\left(H^{1}\left(\Omega^{\varepsilon}\right)\right)^{3}|v|_{r_{d}}=0\right\},
$$

where $\left.v\right|_{\Gamma_{\mathrm{d}}}$ represents the value of $v$ on the boundary $\Gamma_{\mathrm{d}}$ (in the trace sense).

Then the problem of the deformation of a body $\Omega^{\varepsilon}$ subjected to body forces $f$ and tractions $t$ on the boundary $\Gamma_{\mathrm{t}}$ together with tractions $p$ inside the holes $S$, and prescribed displacement on $\Gamma_{\mathrm{d}}$ (with $\left.\Gamma=\Gamma_{\mathrm{t}} \cup \Gamma_{\mathrm{d}}, \Gamma_{\mathrm{t}} \cap \Gamma_{\mathrm{d}}=\emptyset, \Gamma_{\mathrm{d}} \cap S^{\varepsilon}=\emptyset, \Gamma_{\mathrm{t}} \cap S^{\varepsilon}=\emptyset\right)$, can be stated as

Find $u^{\varepsilon} \in V^{\varepsilon}$, such that

$$
\int_{\Omega^{\varepsilon}} E_{i j k l}^{\varepsilon} \frac{\partial u_{k}^{\varepsilon}}{\partial x_{l}} \frac{\partial v_{i}}{\partial x_{j}} \mathrm{~d} \Omega=\int_{\Omega^{\varepsilon}} f_{i}^{\varepsilon} v_{i} \mathrm{~d} \Omega+\int_{\Gamma_{i}} t_{i} v_{i} \mathrm{~d} \Gamma+\int_{S^{\varepsilon}} p_{i}^{\varepsilon} v_{i} \mathrm{~d} S \quad \forall v \in V^{\varepsilon}
$$

Here, it is assumed that the stress-strain and the strain-displacement relations are

$$
\begin{aligned}
& \sigma_{i j}^{\varepsilon}=E_{i j k l}^{\varepsilon} e_{k l}^{\varepsilon}, \\
& e_{k l}^{\varepsilon}=\frac{1}{2}\left(\frac{\partial u_{k}^{\varepsilon}}{\partial x_{l}}+\frac{\partial u_{l}^{\varepsilon}}{\partial x_{k}}\right),
\end{aligned}
$$

and that the elastic constants have the following properties:

$$
\begin{aligned}
& E_{i j k l}^{\varepsilon}=E_{j i k l}^{\varepsilon}=E_{i j l k}^{\varepsilon}=E_{k l i j}^{\varepsilon}, \\
& \exists \alpha>0: E_{i j k l}^{\varepsilon} e_{j l} e_{k l}=\alpha e_{i j} e_{i j} \quad \forall e_{i j}=e_{j l} .
\end{aligned}
$$

A unique solution $u^{b}$ exists for the problem (2.11) under the assumption that the functions $f, t$, and $p$ are sufficiently smooth, and the boundaries $\Gamma_{\mathrm{d}}, \Gamma_{\mathrm{t}}$ and $S^{e}$ are regular, see [14].

Since the body iorces $f$, the tractions $p$ and the elastic constants vary within a small cell of the composite, i.e., they are functions of both $x$ and $y=x / \varepsilon$ :

$$
\Phi^{\varepsilon}(x)=\Phi(x, y), \quad y=x / \varepsilon,
$$

the solution $u_{\varepsilon}$ should also depend both on $x$ and $x / \varepsilon$, that is,

$$
u_{\varepsilon}(x)=u(x, y), \quad y=x / \varepsilon .
$$

Dependence on $y=x / \varepsilon$ means that a quantity varies within a very small region with dimensions much smaller than those of the macroscopic level. In a neighborhood of a macroscopic point $\boldsymbol{x}$ it is assumed that there is a very large number of microscale small cells which are obtained by translation of a base cell. In other words, dependence on $y$ can be considered periodic, specifically $\boldsymbol{Y}$-periodic, for a fixed $\boldsymbol{x}$ in the macroscopic level. Moreover, it is assumed that the form and composition of the base cell varies in a smooth way with the macroscopic variable $\boldsymbol{x}$. This means that for different points $\boldsymbol{x}$ the structure of the composite 
may vary, but if one 'looks through a microscope' on a point $x$, a periodic pattern can be found.

The dependence of the solution $u^{\varepsilon}$ in the macroscopic and microscopic levels makes it reasonable to assume that $\boldsymbol{u}^{\varepsilon}$ can be expresed as an asymptotic expansion with respect to the parameter $\varepsilon$ (a measure of the microscopic/macroscopic dimension ratio), i.e.,

$$
u^{\varepsilon}(x)=u^{0}(x, y)+\varepsilon u^{1}(x, y)+\varepsilon^{2} u^{2}(x, y)+\cdots, \quad y=x / \varepsilon,
$$

where

$$
\begin{aligned}
& u^{j}(x, y) \text { is defined in }(x, y) \in \Omega \times \Psi, \\
& y \rightarrow u^{j}(x, y) \text { is } Y \text {-periodic . }
\end{aligned}
$$

To establish equations which $u^{0}, u^{1}, \ldots, u^{j}$ satisfy, it is useful to note that

$$
\frac{\partial}{\partial x_{i}}(\Phi(x, y=x / \varepsilon))=\frac{\partial \Phi}{\partial x_{i}}+\frac{1}{\varepsilon} \frac{\partial \Phi}{\partial y_{i}}
$$

and that, for a $Y$-periodic function $\Psi(y)$,

$$
\begin{aligned}
& \lim _{\left\{\rightarrow 0^{+}\right.} \int_{\Omega^{\mathrm{e}}} \Psi\left(\frac{x}{\varepsilon}\right) \mathrm{d} \Omega \rightarrow \frac{1}{|\boldsymbol{Y}|} \int_{\Omega} \int_{V} \Psi(y) \mathrm{d} Y \mathrm{~d} \Omega, \\
& \lim _{\varepsilon \rightarrow 0^{+}} \varepsilon \int_{S^{\varepsilon}} \Psi\left(\frac{x}{\varepsilon}\right) \mathrm{d} S \rightarrow \frac{1}{|Y|} \int_{\Omega} \int_{S} \Psi(y) \mathrm{d} S \mathrm{~d} \Omega,
\end{aligned}
$$

where $|Y|$ stands for the volume (or area, for two dimensional domain) of the cell. Implicitly it is assumed that the function $\Psi(y)$ is extended to all volume under each cell, such that it takes the value zero on the holes. This will be assumed to be possible in a smooth way. In the following description it will be assumed that all functions depend explicitly both on $x$ and $y$ with a single exception for the applied traction $t$ on boundary $\Gamma_{t}$, which only depends on $x$. When a particular dependence on $x$ or $y$ is assumed, it will explicitly be stated in the equations.

Define

$$
\begin{aligned}
& V_{\Omega \times Y}=\left\{v(x, y) \text { defined for }(x, y) \in \Omega \times Y \mid v(\cdot, y) Y \text {-periodic; }\left.v\right|_{\Gamma_{\mathrm{d}}}=0 ;\right. \\
& \quad v \text { smooth enough }\} \\
& V_{\Omega}=\left\{v(x) \text { defined in } \Omega|v|_{\Gamma_{\mathrm{d}}}=0 ; v \text { smooth enough }\right\} \\
& V_{*}=\{v(y) \text { defined in } ¥ \mid v(y) Y \text {-periodic; } v \text { smooth enough }\} .
\end{aligned}
$$

Introducing the asymptotic expansion (2.18) and (2.20) into (2.11), the functional becomes 


$$
\begin{aligned}
\int_{\Omega^{\varepsilon}} E_{i j k l}^{\varepsilon}\left\{\frac{1}{\varepsilon^{2}} \frac{\partial u_{k}^{0}}{\partial y_{l}} \frac{\partial v_{i}}{\partial y_{j}}+\frac{1}{\varepsilon}\left[\left(\frac{\partial u_{k}^{0}}{\partial x_{l}}+\frac{\partial u_{k}^{l}}{\partial y_{l}}\right) \frac{\partial v_{i}}{\partial y_{j}}+\frac{\partial u_{k}^{0}}{\partial y_{l}} \frac{\partial v_{i}}{\partial x_{j}}\right]\right. \\
\left.\quad+\left[\left(\frac{\partial u_{k}^{0}}{\partial x_{l}}+\frac{\partial u_{k}^{1}}{\partial y_{l}}\right) \frac{\partial v_{i}}{\partial x_{j}}+\left(\frac{\partial u_{k}^{1}}{\partial x_{l}}+\frac{\partial u_{k}^{2}}{\partial y_{l}}\right) \frac{\partial v_{i}}{\partial y_{j}}\right]+\varepsilon(\cdots)\right\} \mathrm{d} \Omega \\
=\int_{\Omega^{\varepsilon}} f_{i}^{\varepsilon} v_{i} \mathrm{~d} \Omega+\int_{\Gamma_{t}} t_{i} v_{i} \mathrm{~d} \Gamma+\int_{S^{\varepsilon}} p_{i}^{\varepsilon} v_{i} \mathrm{~d} S \quad \forall v \in V_{\Omega \times Y}
\end{aligned}
$$

Assuming that the functions are smooth enough so that the limit when $\varepsilon \rightarrow 0^{+}$of all integrals exists, (2.26) holds if the terms of the same power of $\varepsilon$ are equal to zero. Therefore

$$
\begin{aligned}
& \frac{1}{\varepsilon^{2}} \int_{\Omega^{\varepsilon}} E_{i j k l}^{s} \frac{\partial u_{k}^{0}}{\partial y_{l}} \frac{\partial v_{i}}{\partial y_{j}} \mathrm{~d} \Omega=0 \quad \forall v \in V_{\Omega \times Y} \\
& \frac{1}{\varepsilon} \int_{\Omega^{\varepsilon}} E_{i j k l}^{\varepsilon}\left[\left(\frac{\partial u_{k}^{0}}{\partial x_{l}}+\frac{\partial u_{k}^{1}}{\partial y_{l}}\right) \frac{\partial v_{i}}{\partial y_{j}}+\frac{\partial u_{k}^{0}}{\partial y_{l}} \frac{\partial v_{i}}{\partial x_{j}}\right] \mathrm{d} \Omega=\int_{S^{\varepsilon}} p_{i}^{\varepsilon} v_{i} \mathrm{~d} S \quad \forall v \in V_{\Omega \times Y}, \\
& \int_{\Omega^{\varepsilon}} E_{i j k l}^{\varepsilon}\left[\left(\frac{\partial u_{k}^{0}}{\partial x_{l}}+\frac{\partial u_{k}^{1}}{\partial y_{l}}\right) \frac{\partial v_{i}}{\partial x_{j}}+\left(\frac{\partial u_{k}^{1}}{\partial x_{l}}+\frac{\partial u_{k}^{2}}{\partial y_{l}}\right) \frac{\partial v_{i}}{\partial y_{j}}\right] \mathrm{d} \Omega=\int_{\Omega^{\varepsilon}} f_{i}^{\varepsilon} v_{i} \mathrm{~d} \Omega+\int_{\Gamma_{t}} t_{i} v_{i} \mathrm{~d} \Gamma \\
& \forall v \in V_{\Omega \times Y} \\
& \text { etc. }
\end{aligned}
$$

Multiplying (2.27) by $\varepsilon^{2}$ and taking the limit $\varepsilon \rightarrow 0^{+}$, property (2.21) yields

$$
\frac{1}{|Y|} \int_{\Omega} \int_{V} E_{i j k l} \frac{\partial u_{k}^{0}}{\partial y_{l}} \frac{\partial v_{i}}{\partial y_{j}} \mathrm{~d} Y \mathrm{~d} \Omega=0 \quad \forall v \in V_{\Omega \times Y}
$$

Since $v$ is arbitrary, choose $v=v(y)$, i.e., $v \in V_{v}$. Then, integrating by parts, applying the divergence theorem to the integral in $Y$, and noting that the terms in the opposite faces of the cube $\boldsymbol{Y}$ cancel due to the periodicity condition, one has

$$
\frac{1}{|Y|} \int_{\Omega}\left\{\int_{Y}\left[-\frac{\partial}{\partial y_{j}}\left(E_{i j k l} \frac{\partial u_{k}^{0}}{\partial y_{l}}\right)\right] v_{i} \mathrm{~d} Y+\int_{S} E_{i j k l} \frac{\partial u_{k}^{0}}{\partial y_{l}} n_{j} v_{i} \mathrm{~d} S\right\} \mathrm{d} \Omega=0 \quad \forall v \in V_{\Omega \times Y}
$$

Since $v(y)$ is arbitrary, this yields the boundary value problem of the first term $u^{0}$ of the expansion of the original solution $\boldsymbol{u}^{\varepsilon}$ in the basic cell dornain $Y$ :

$$
\begin{aligned}
& -\frac{\partial}{\partial y_{j}}\left(E_{i j k l} \frac{\partial u_{k}^{0}}{\partial y_{l}}\right)=0, \quad y \in Y, \\
& E_{i j k l} \frac{\partial u_{k}^{0}}{\partial y_{l}} n_{j}=0 \text { on } S .
\end{aligned}
$$


Apply the following proposition, which is identical to Lemma 2.1 in [2, p. 10].

PROPOSITION 1. The problem

$$
\frac{\partial}{\partial y_{j}}\left(E_{i j k l}(y) \frac{\partial \Phi(y)}{\partial y_{l}}\right)=F(y), \quad y \in \Psi
$$

has a solution in

$$
V_{\boldsymbol{V}}=\{\boldsymbol{v} \text { smooth enough, } \boldsymbol{v} \text { is } Y \text {-periodic }\}
$$

defined up to an additive constant, for a regular $F$, if and only if

$$
\int_{Y} F(y) \mathrm{d} Y=-\int_{S} E_{i j k l} \frac{\partial \Phi(y)}{\partial y_{l}} n_{j} \mathrm{~d} S .
$$

Then it follows from (2.32) and (2.33) that

$$
u^{0}=u^{0}(x),
$$

that is, the first term of the expansion of $\boldsymbol{u}^{8}$ in $\varepsilon$ depends only on the macroscopic scale $x$.

Introducing (2.36) into (2.28), multiplying by $\varepsilon$, taking the limit $\varepsilon \rightarrow 0^{+}$and using properties (2.21) and (2.22), one can obtain

$$
\begin{aligned}
& \int_{\Omega}\left[\frac{1}{|Y|} \int_{V} E_{i j k l}\left(\frac{\partial u_{k}^{0}(x)}{\partial x_{l}}+\frac{\partial u_{k}^{1}}{\partial y_{l}}\right) \frac{\partial v_{i}}{\partial y_{j}} \mathrm{~d} Y\right] \mathrm{d} \Omega=\int_{\Omega}\left(\frac{1}{|Y|} \int_{S} p_{i} v_{i} \mathrm{~d} S\right) \mathrm{d} \Omega \\
& \forall v \in V_{\Omega \times Y}
\end{aligned}
$$

Since (2.37) is satisfied for any $v$, choosing $v=v(y)$ yields

$$
\int_{Y} E_{i j k l}\left(\frac{\partial u_{k}^{0}(x)}{\partial x_{l}}+\frac{\partial u_{k}^{1}}{\partial y_{l}}\right) \frac{\partial v_{i}(y)}{\partial y_{j}} \mathrm{~d} Y=\int_{S} p_{i} v_{i}(y) \mathrm{d} S \quad \forall v \in V_{Y} .
$$

Integrating by parts, using the divergence theorem, and applying the periodicity conditions on the opposite faces of $Y$, it follows from (2.38) that

$$
\begin{aligned}
& -\int_{Y} \frac{\partial}{\partial y_{j}}\left[E_{i j k l}\left(\frac{\partial u_{k}^{0}(x)}{\partial x_{l}}+\frac{\partial u_{k}^{1}}{\partial y_{l}}\right)\right] v_{i}(y) \mathrm{d} Y+\int_{S} E_{i j k l}\left(\frac{\partial u_{k}^{0}(x)}{\partial x_{l}}+\frac{\partial u_{k}^{1}}{\partial y_{l}}\right) v_{i}(y) n_{j} \mathrm{~d} S \\
& \quad=\int_{S} p_{i} v_{i}(y) \mathrm{d} S \quad \forall v \in V_{Y} .
\end{aligned}
$$

Since $v$ is arbitrary, 


$$
\begin{aligned}
& -\frac{\partial}{\partial y_{j}}\left(E_{i j k l} \frac{\partial u_{k}^{1}}{\partial y_{l}}\right)=\frac{\partial}{\partial y_{j}}\left(E_{i j k l} \frac{\partial u_{k}^{0}(x)}{\partial x_{l}}\right) \text { on } \Psi, \\
& E_{i j k l} \frac{\partial u_{k}^{1}}{\partial y_{l}} n_{j}=-E_{i j k l} \frac{\partial u_{k}^{0}(x)}{\partial x_{l}} n_{j}+p_{i} \text { on } S .
\end{aligned}
$$

On the other hand, if in (2.37) $v$ is chosen to be only a function of $x$, i.e., $v=v(x)$, then

$$
\int_{\Omega}\left(\frac{1}{|Y|} \int_{S} p_{i} \mathrm{~d} S\right) v_{i}(x) \mathrm{d} \Omega=0 \quad \forall v \in V_{\Omega},
$$

which implies that

$$
\int_{S} p_{i}(x, y) \mathrm{d} S=0 .
$$

Equation (2.43) restricts the possible applied tractions inside the holes. Moreover, if in (2.42) the set $V_{\Omega}$ is relaxed in order to contain all rigid body rotations, it can be seen that the moment in each cell due to the tractions $p$ has to be zero. That is, the applied tractions have to be self-equilibrating. Thus, it follows from Proposition 1 that there is a solution $u^{1} \in V_{v}$ defined up to a constant.

Introducing (2.36) into (2.29) and taking the limit $\varepsilon \rightarrow 0^{+}$in (2.29) implies

$$
\begin{gathered}
\int_{\Omega}\left\{\frac{1}{|Y|} \int_{V} E_{i j k l}\left[\left(\frac{\partial u_{k}^{0}}{\partial x_{l}}+\frac{\partial u_{k}^{1}}{\partial y_{l}}\right) \frac{\partial v_{i}}{\partial x_{j}}+\left(\frac{\partial u_{k}^{1}}{\partial x_{l}}+\frac{\partial u_{k}^{2}}{\partial y_{l}}\right) \frac{\partial v_{l}}{\partial y_{j}}\right] \mathrm{d} Y\right\} \mathrm{d} \Omega \\
=\int_{\Omega}\left(\frac{1}{|Y|} \int_{V} f_{i} v_{i} \mathrm{~d} Y\right) \mathrm{d} \Omega+\int_{\Gamma_{i}} t_{i} v_{i} \mathrm{~d} \Gamma \quad \forall v \in V_{\Omega \times V} .
\end{gathered}
$$

Choosing $v=v(x)$ yields

$$
\begin{aligned}
& \int_{\Omega} {\left[\frac{1}{|Y|} \int_{Y} E_{i j k l}\left(\frac{\partial u_{k}^{0}}{\partial x_{l}}+\frac{\partial u_{k}^{1}}{\partial y_{l}}\right) \mathrm{d} Y\right] \frac{\partial v_{i}(x)}{\partial x_{j}} \mathrm{~d} \Omega } \\
& \quad=\int_{\Omega}\left(\frac{1}{|Y|} \int_{Y} f_{i} \mathrm{~d} Y\right) v_{i}(x) \mathrm{d} \Omega+\int_{\Gamma_{i}} t_{i} v_{i}(x) \mathrm{d} \Gamma \quad \forall v \in V_{\Omega} .
\end{aligned}
$$

Note that this is a statement of overall equilibrium in the nacroscopic sense.

Now if $v=v(y)$ is assumed in (2.44), then

$$
\begin{aligned}
& \int_{\Omega}\left[\frac{1}{|Y|} \int_{Y} E_{i j k l}\left(\frac{\partial u_{k}^{1}}{\partial x_{l}}+\frac{\partial u_{k}^{2}}{\partial y_{l}}\right) \frac{\partial v_{i}(y)}{\partial y_{j}} \mathrm{~d} Y\right] \mathrm{d} \Omega=\int_{\Omega}\left(\frac{1}{|Y|} \int_{Y} f_{i} v_{i}(y) \mathrm{d} Y\right) \mathrm{d} \Omega \\
& \forall v \in V_{Y} .
\end{aligned}
$$

This is equivalent to 


$$
\int_{Y} E_{i j k l}\left(\frac{\partial u_{k}^{1}}{\partial x_{l}}+\frac{\partial u_{k}^{2}}{\partial y_{l}}\right) \frac{\partial v_{i}(y)}{\partial y_{j}} \mathrm{~d} Y=\int_{Y} f_{i} v_{i}(y) \mathrm{d} Y \quad \forall v \in V_{Y}
$$

which represents microscopic equilibrium in the basic cell.

The same procedure could be applied for the higher order terms of the expansion, and then a set of 'equilibrium' equations relating the several terms would be obtained. However, if only the first order terms are of interest, solving (2.38) and (2.45) yields the 'full' solution for $\boldsymbol{u}^{\varepsilon}$. The term $u^{0}$ represents, essentially, the macroscopic mechanical behavior while the $u^{1}$ represents the microscopic behavior.

The goal now is to find homogenized elastic constants such that the macroscopic equilibrium can be described by the same sort of equation as (2.11). These homogenized constants should be such that the corresponding equilibrium equation reflects the mechanical behavior of the microstructure of the composite material without explicitly using the microscale parameter $\varepsilon$. To accomplish this consider once again (2.38). It can easily be recognized that this equation is linear with respect to $u^{0}$ and $p$. This suggests considering the two following problems:

Let $\chi^{k l} \in V_{v}$ be the solution of

$$
\int_{Y} E_{i j p m} \frac{\partial \chi_{p}^{k l}}{\partial y_{m}} \frac{\partial v_{i}(y)}{\partial y_{j}} \mathrm{~d} Y=\int_{V} E_{i j k l} \frac{\partial v_{i}(y)}{\partial y_{j}} \mathrm{~d} Y \quad \forall v \in V_{Y}
$$

and let $\psi \in V_{\psi}$ be the solution of

$$
\int_{V} E_{i j k l} \frac{\partial \psi_{k}}{\partial y_{l}} \frac{\partial v_{i}(y)}{\partial y_{j}} \mathrm{~d} Y=\int_{S} p_{i} v_{l}(y) \mathrm{d} Y \quad \forall v \in V_{\boldsymbol{V}},
$$

where $\boldsymbol{x}$ plays the role of a parameter.

The existence of solutions to these problems, calculated to within additive constants, is assured by Proposition 1. The additive constants may be functions of $x$. Therefore, due to the linearity of the problem (2.38), the solution $u^{1}$ can be written as

$$
u_{i}^{1}=-\chi_{i}^{k l}(x, y) \frac{\partial u_{k}^{0}(x)}{\partial x_{l}}-\psi_{i}(x, y)+\tilde{u}_{i}^{1}(x),
$$

where $\tilde{u}_{i}^{1}$ are arbitrary additive constants in $y$. Introducing (2.50) into (2.45) yields

$$
\begin{aligned}
& \int_{\Omega}\left[\frac{1}{|Y|} \int_{V}\left(E_{i j k l}-E_{i j p m} \frac{\partial x_{p}^{k l}}{\partial y_{m}}\right) \mathrm{d} Y\right] \frac{\partial u_{k}^{0}(x)}{\partial x_{l}} \frac{\partial v_{i}(x)}{\partial x_{j}} \mathrm{~d} \Omega \\
& =\int_{\Omega}\left(\frac{1}{|Y|} \int_{V} E_{i j k l} \frac{\partial \psi_{k}}{\partial y_{l}} \mathrm{~d} Y\right) \frac{\partial v_{i}(x)}{\partial x_{j}} \mathrm{~d} \Omega+\int_{\Omega}\left(\frac{1}{|Y|} \int_{Y} f_{i} \mathrm{~d} Y\right) v_{i}(x) \mathrm{d} \Omega \\
& \quad+\int_{\Gamma_{t}} t_{i} v_{i}(x) \mathrm{d} \Gamma \quad \forall v \in V_{\Omega} .
\end{aligned}
$$

Denoting 
and

$$
\begin{aligned}
& D_{i j k l}(x)=\frac{1}{|Y|} \int_{Y}\left(E_{i j k l}-E_{i j p m} \frac{\partial \chi_{p}^{k l}}{\partial y_{m}}\right) \mathrm{d} Y, \\
& \tau_{i j}(x)=\frac{1}{|Y|} \int_{Y} E_{i j k l} \frac{\partial \psi_{k}}{\partial y_{l}} \mathrm{~d} Y
\end{aligned}
$$

$$
b_{i}(x)=\frac{1}{|Y|} \int_{Y} f_{i} \mathrm{~d} Y \text {, }
$$

(2.51) can be written as

$$
\begin{aligned}
& \int_{\Omega} D_{i j k l}(x) \frac{\partial u_{k}^{0}(x)}{\partial x_{l}} \frac{\partial v_{i}(x)}{\partial x_{j}} \mathrm{~d} \Omega \\
& \quad=\int_{\Omega} \tau_{i j}(x) \frac{\partial v_{i}(x)}{\partial x_{j}} \mathrm{~d} \Omega+\int_{\Omega} b_{i}(x) v_{i}(x) \mathrm{d} \Omega+\int_{\Gamma_{i}} t_{i}(x) v_{i}(x) \mathrm{d} \Gamma \quad \forall v \in V_{\Omega} .
\end{aligned}
$$

This equation represents the macroscopic equilibrium, while $D_{i j k l}$ defined by (2.52) represents the homogenized elastic constants, $\tau_{i j}$ an average 'residual' stress within the cell due to the tractions $p$ inside the holes and $b_{i}$ and average body force.

As shown above, the microscopic and macroscopic problems are not coupled, i.e., the homogenized elastic constants can be computed within the basic cell by solving problems (2.48) and (2.49), which do not depend on the macroscopic deformation $u^{0}$. If a composite material has a uniform cell structure in the whole domain $\Omega$, as well as a uniform applied traction $p$ on the hole boundaries of the cells, then the microscopic problems (2.48) and (2.49) need to be solved only once. If they are not uniform in the domain $\Omega$, these microscopic problems must be solved for every point $x$ of $\Omega$ at which cell structure and traction $p$ are different from others.

The following remarks could be useful to understand the homogenization method.

REMARK 2.1. The above derivations are formal, and all functions are assumed to be smooth enough in both $\boldsymbol{x}$ and $\boldsymbol{y}$.

REMARK 2.2. The assumption that the holes of the composite cells do not intersect the boundary $\Gamma$ of the domain $\Omega$, made it easier to deal with the boundary conditions. A more careful treatment of the boundary requires the use of boundary layer terms, in particular, to take care of term $\tilde{u}^{1}$, see $[2,4]$.

REMARK 2.3. From problem (2.48) and property (2.14) of the elastic constants, it is easily seen that $\chi^{k l}$ is symmetric with respect to the indices $k$ and $l$. This implies that only six different equations are solved for the three-dimensional problem (and only three for the two-dimensional problem).

REMARK 2.4. From (2.52), it can be shown that the symmetry property and coercivity also hold for the homogenized elastic constants, i.e., 


$$
\begin{aligned}
& D_{i j k l}=D_{i j l k}=D_{j i k l}=D_{k l i j}, \\
& \exists \alpha>0: D_{i j k l} e_{i j} e_{k l} \geqslant \alpha e_{i j} e_{i j} \quad \forall e_{i j}=e_{j i} .
\end{aligned}
$$

Details of the proofs can be found in [6].

REMARK 2.5. It can be shown that under smoothness assumption on $\tau, b$ and $t$, the homogenized problem has a unique solution $u^{0}$, see [14].

REMARK 2.6. It is assumed that the solution $u^{\varepsilon}$ converges (weakly) to $u^{0}$ whenever $\varepsilon \rightarrow 0^{+}$. Proofs and detailed analysis of such convergence can be found in $[15,16]$.

REMARK 2.7. The displacement field $u^{\varepsilon}$ involving details of the microstructure is given by

$$
u^{\varepsilon}(x)=u^{0}(x)-\varepsilon\left(x^{k l}(x, y) \frac{\partial u_{k}^{0}(x)}{\partial x_{l}}+\psi(x, y)-\tilde{u}^{1}(x)\right)+\varepsilon^{2}(\cdots), \quad y=\frac{x}{\varepsilon}
$$

once the homogenized macroscopic problem is solved.

REMARK 2.8. The stresses in each point of the domain are given by

$$
\sigma_{i j}^{\varepsilon}=E_{i j k l} \frac{\partial u_{k}^{\varepsilon}}{\partial x_{l}},
$$

substituting (2.58)

where

$$
\sigma_{i j}^{\varepsilon}=\sigma_{i j}^{0}+\varepsilon \sigma_{i j}^{1}+\varepsilon^{2}(\cdots),
$$

$$
\begin{aligned}
& \sigma_{i j}^{0}=E_{i j k l}\left(\frac{\partial u_{k}^{0}}{\partial x_{l}}+\frac{\partial u_{k}^{1}}{\partial y_{l}}\right), \\
& \sigma_{i j}^{1}=E_{i j k l}\left(\frac{\partial u_{k}^{1}}{\partial x_{l}}+\frac{\partial u_{k}^{2}}{\partial y_{l}}\right),
\end{aligned}
$$

Then introducing (2.50) into $(2.60), \sigma_{i j}^{0}$, the first approximation of the stress, is given by

$$
\begin{aligned}
& \sigma_{i j}^{0}(x, y)=\left(E_{i j k l}(x, y)-E_{i j p m}(x, y) \frac{\partial \chi_{p}^{k l}(x, y)}{\partial y_{m}}\right) \frac{\partial u_{k}^{0}(x)}{\partial x_{l}}-E_{i j k l}(x, y) \frac{\partial \psi_{k}(x, y)}{\partial x_{l}}, \\
& y=\frac{x}{\varepsilon}
\end{aligned}
$$

REMARK 2.9. The relation between the homogenized stress $\sigma_{i j}^{\mathrm{h}}=D_{i j k l} \partial u_{k}^{0} / \partial x_{l}$ and $\sigma_{i j}^{0}$ can readily be seen by applying the 'average' operator 


$$
\frac{1}{|Y|} \int_{Y} \cdots \mathrm{d} Y
$$

to the previous definition to obtain

$$
\sigma_{i j}^{\mathrm{h}}=\frac{1}{|Y|} \int_{\mathbf{Y}} \sigma_{i j}^{0} \mathrm{~d} Y+\tau_{i j}
$$

REMARK 2.10. If there are no applied tractions $t$ and body forces $f$, it follows from (2.55) that

and then

$$
\left(\sigma_{i j}^{\mathrm{h}}-\tau_{i j}\right)_{, j}=0 \text { in } \Omega, \quad\left(\sigma_{i j}^{\mathrm{h}}-\tau_{i j}\right) n_{j}=0 \text { on } \Gamma_{\mathrm{t}},
$$

$$
\sigma_{i j}^{\mathrm{h}}=\tau_{i j} \text {. }
$$

REMARK 2.11. For the particular case that either there are no applied tractions $p$ on the holes $S$ or the holes do not exist, we set $\psi=0$ and $Y=Y$.

Up to this point derivations of equations were introduced characterizing the homogenization method. All these derivations were performed assuming enough smoothness for all functions and functionals involved. The homogenization method was then characterized by the solution of three distinct problems: two in the microscopic level, cell problerns, and the other in the macroscopic level. The notation involved in characterizing these problems was based on the principle of virtual work, that is, the weak form of the equilibrium equations, in order to present the homogenization results in a more familiar way. However, this notation is not very convenient for the following developments. Also a more precise mathematical setting is necessary to formulate the problems. Consequently a new notation is now introduced, as well as the required mathematical framework.

Let $\left(H^{m}(\Omega)\right)^{3}$ and $\left(H^{m}(\mathcal{Y})\right)^{3}$ be Sobolev spaces defined over the $\mathbb{R}^{3}$ domains $\Omega$ and $\boldsymbol{Y}$, respectively, with the semi-norm and norm

and

$$
|v|_{r, \Omega}=\left(\sum_{|\alpha|=r} \int_{\Omega}\left|\partial^{\alpha} v_{1}\right|^{2}+\left|\partial^{\alpha} v_{2}\right|^{2}+\left|\partial^{\alpha} v_{3}\right|^{2} \mathrm{~d} \Omega\right)^{1 / 2}, 0 \leqslant r \leqslant m
$$

$$
\|v\|_{m, \Omega}=\left(\sum_{|\alpha|<m} \int_{\Omega}\left|\partial^{\alpha} v_{1}\right|^{2}+\left|\partial^{\alpha} v_{2}\right|^{2}+\left|\partial^{\alpha} v_{3}\right|^{2} \mathrm{~d} \Omega\right)^{1 / 2},
$$

respectively, where $\alpha$ is a multi-index $\left(\alpha_{1}, \alpha_{2}, \alpha_{3}\right)$ and

$$
\partial^{\alpha} g=\frac{\partial^{|\alpha|} g}{\partial^{\alpha_{1}} x_{1} \partial^{\alpha_{2}} x_{2} \partial^{\alpha_{3}} x_{3}}, \quad|\alpha|=\alpha_{1}+\alpha_{2}+\alpha_{3} .
$$

Similar norms and semi-norms are defined for the spaces $\left(H^{m}(\eta)\right)^{3}$. Now redefine the set $V_{y}$ as

$$
V_{Y}=\left\{v \in\left(H^{1}(Y)\right)^{3} \mid v \text { is } Y \text {-periodic }\right\},
$$


introduce the bilinear form defined on $V_{y} \times V_{y}$,

$$
a_{Y}(u, v)=\frac{1}{|Y|} \int_{Y} E_{i j k l} \frac{\partial u_{k}}{\partial y_{l}} \frac{\partial v_{i}}{\partial y_{j}} \mathrm{~d} Y, \quad u, v \in V_{Y},
$$

and the linear functional in $V_{\mathbf{z}}$,

$$
f_{Y}(v)=\frac{1}{|Y|} \int_{S} p_{i} v_{i} \mathrm{~d} S
$$

and define

$$
P^{i j}=y_{j} \delta_{i k} e_{k},
$$

where $e_{k}$ are the Cartesian base vectors for $\mathbb{R}^{3}$ and $\delta_{i k}$ is the Kronecker symbol. Then one can restate problem $(2.48)$ as

Find $x^{k l} \in V_{y}$, such that

$$
a_{Y}\left(X^{k l}-P^{k l}, v\right)=0 \quad \forall v \in V_{v} .
$$

A direct substitution yields that the homogenized coefficients (2.52) can be written as

$$
D_{i j k l}(x)=a_{Y}\left(P^{k l}-\chi^{k l}, P^{i j}\right)
$$

or, choosing $v=\chi^{\prime \prime}$ in (2.70) and adding to (2.71) yields

$$
D_{i j k l}(x)=a_{Y}\left(P^{k l}-\chi^{k l}, P^{i j}-\chi^{i j}\right) .
$$

Similarly, problem $(2.49)$ is represented by

Find $\psi \in V_{w}$, such that

$$
a_{Y}(\psi, v)=f_{Y}(v), \quad \forall v \in V_{Y} .
$$

The 'residual' stresses $\tau$ can then be expressed by

$$
\tau_{i j}=a_{Y}\left(\psi, P^{i j}\right) .
$$

Finally, for the macroscopic problem, define the set

$$
V_{\Omega}=\left\{v \in\left(H^{1}(\Omega)\right)^{3}|v|_{r_{d}}=0\right\},
$$

and introduce the bilinear form in $V_{\Omega} \times V_{\Omega}$ 


$$
a(u, v)=\int_{\Omega} D_{i j k l} \frac{\partial u_{k}}{\partial x_{l}} \frac{\partial v_{i}}{\partial x_{j}} \mathrm{~d} Y, \quad u, v \in V_{\Omega}
$$

and the linear functional in $V_{\Omega}$,

$$
f(v)=\int_{\Omega} \tau_{i j} \frac{\partial v_{i}}{\partial x_{j}} \mathrm{~d} \Omega+\int_{\Omega} b_{i} v_{i} \mathrm{~d} \Omega+\int_{\Gamma_{\mathrm{t}}} t_{i} v_{i} \mathrm{~d} \Gamma
$$

Then (2.55) is written as

Find $u^{0} \in V_{\Omega}$, such that

$$
a\left(u^{0}, v\right)=f(v) \quad \forall v \in V_{\Omega}
$$

$\operatorname{REMARK} 2.12$. The bilinear forms $a_{Y}(\cdot, \cdot)$ and $a(\cdot, \cdot)$ are symmetric.

REMARK 2.13. It can be shown that $a_{Y}(\cdot, \cdot)$ defines a semi-norm in the space $V_{Y}$. In particular due to the properties of the elastic coefficients $E_{i j k l}$ it defines a semi-norm equivalent to the $\mid \cdot l_{1, y}$, i.e.,

$$
\exists \alpha>0, \beta>0: \alpha|v|_{1, Y} \leqslant a_{Y}(v, v)^{1 / 2} \leqslant \beta|v|_{1, Y} \quad \forall v \in V_{Y}
$$

REMARK 2.14. If one defines the quotient space $V^{\bullet}=V_{y} /\left(\mathbb{R}^{3}\right)$, i.e., the space of all equivalent classes $[u]$ such that $(u-v) \in \mathbb{R}^{3}, u, v \in V_{v}$ with the usual norm definition

$$
\|[u]\|=\inf _{c \in \mathbb{R}^{3}}\|u-c\|_{1, v}
$$

the cell problems (2.70) and (2.73) have unique solutions in $V^{\bullet}$ (this is a simple extension of Lemma 2.1 in [2]).

REMARK 2.15. The norm in $V^{\bullet}$ is equivalent to the semi norm $|\cdot|_{1, v}$ (see [17]), and consequently equivalent to the bilinear form $a_{Y}(\cdot, \cdot)$.

REMARK 2.16. It can be shown that $a_{Y}(\cdot, \cdot)$ satisfies a Schwarz inequality, i.e.,

$$
\left|a_{Y}(u, v)\right| \leqslant a_{Y}(u, u)^{1 / 2} a_{Y}(v, v)^{1 / 2}, \quad u, v \in V_{Y} .
$$

REMARK 2.17. Under the properties (2.56), (2.57) of the homogenized coefficients and the assumption that the bilinear form $(\cdot, \cdot)$ satisfies a Korn inequality (see [14]), a( $\left.\cdot,^{\cdot}\right)$ defines an equivalent norm to $\|\cdot\|_{1, \Omega}$ in $V_{\Omega}$, i.e.,

$$
\exists \alpha, \beta>0: \alpha\|v\|_{1, \Omega} \leqslant a(v, v)^{1 / 2} \leqslant \beta\|v\|_{1, \Omega} \quad \forall v \in V_{\Omega}
$$

REMARK 2.18. It can be shown that $a(\cdot, \cdot)$ satisfies a Schwarz inequality, i.e.,

$$
|a(u, v)| \leqslant a(u, u)^{1 / 2} a(v, v)^{1 / 2}, \quad u, v \in V_{\Omega}
$$


REMARK 2.19. The bilinear form $a(\cdot, \cdot)$ and the linear functional $f(\cdot)$ are expressed using the homogenized coefficients and the 'residual' stresses which are obtained in the cell associated with a point $\boldsymbol{x}$ as a parameter.

REMARK 2.20. $P^{i j}$ in (2.69) is not an element of $V_{\mathbf{Y}}$.

\section{Finite element analysis for the homogenization method}

In this section a description is presented of the finite element solution procedure to obtain the homogenized elastic coefficients $D_{i j k l}$, the 'residual' stress $\tau_{i j}$, and the macroscopic displacement field $u^{0}$ of the homogenized problem. For each of these quantities a priori error estimations are obtained. To this end let us assume that

1. Domains $\Omega$ and $\boldsymbol{Y}$ are polygonal;

2. Discretization using regular families of conforming finite elements ;

3. The integration is performed exactly ;

4. A generic space $V$ is approximated by a space $V^{h}$ s.t.

$$
\begin{aligned}
& \operatorname{dim} V^{h}<\infty, \\
& V \supset V^{h}, \\
& \forall v \in V \quad \exists v^{h} \in V^{h}: \lim _{h \rightarrow 0}\left\|v-v^{h}\right\|=0,
\end{aligned}
$$

where the parameter $h$ represents a geometrical sizing characteristic of the finite elements discretizing the domain (see [18]). More specifically, following Babuška and Aziz [19, Chapter

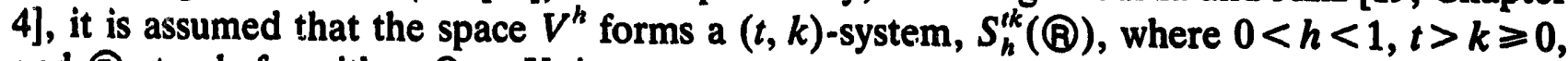
and $(B)$ stands for either $\Omega$ or $\boldsymbol{Y}$, i.e.,

$$
\begin{aligned}
& \left(H^{k}(\circledast)^{3} \supset S_{h}^{t k}(\Theta),\right. \\
& \forall v \in\left(H^{\prime}(囚)^{3}, l \geqslant 0,0 \leqslant s \leqslant \min (l, k) \quad \exists v_{1} \in S_{h}^{t k}(\circledast):\right. \\
& \left\|v-v_{1}\right\|_{s, \circledast} \leqslant C_{1} h^{\mu}\|v\|_{l, \circledast}, \\
& \quad \mu=\min (t-s, l-s),
\end{aligned}
$$

where $C_{1}$ is independent of $v$ and $h, v_{1}$ depends on $v$ and $s$. It is also assumed that $S_{h}^{t k}(囚)$ is regular, i.e., $v_{I}$ is independent of $s$, and $S_{h}^{t k}(\mathbb{B})$ satisfies an inverse relation:

$$
\exists k \geqslant \varepsilon>0: \forall k-\varepsilon \leqslant s \leqslant k \text { and } \forall v \in S_{h}^{t k}(囚) \quad\|v\|_{\mathrm{k}, \circledast} \leqslant C_{2} h^{-(k-s)}\|v\|_{s, \circledast} \text {, }
$$


where $C_{2}$ is independent on $v$ and $h$, but not on $k$ and $\varepsilon$. Using these assumptions it is shown in $[19$, Theorem 4.1 .5$]$ that

$$
\forall v \in\left(H^{l}(\circledast)\right)^{3} \quad \exists v_{1} \in S_{h}^{t k}(\Theta): \forall-t \leqslant s \leqslant k, s \leqslant l \quad\left\|v-v_{l}\right\|_{s, \circledast} \leqslant C h^{\mu}\|v\|_{l, \circledast},
$$

where $C$ is independent of $v$ and $h$, and $v_{I}$ of $s$. It is noted that the norm of the intermediate spaces $\left(H^{s}(\circledR)\right)^{3}$, where $s$ is a real number different from 1 or 2 , is defined by

$$
\begin{aligned}
& \|v\|_{s, \Theta}^{2}=\|v\|_{[s], \circledast}^{2}+\sum_{|\alpha|=[s]}\left\|\partial^{\alpha} v\right\|_{\sigma, \circledast}^{2}, \\
& \|v\|_{\sigma, \circledast}^{2}=\int_{\circledast} \int_{\circledast} \frac{(v(x)-v(\xi)) \cdot(v(x)-v(\xi))}{(x-\xi) \cdot(x-\xi)^{n / 2+\sigma}} \mathrm{d} x \mathrm{~d} \xi,
\end{aligned}
$$

where $[s]$ is the largest integer less or equal than $s$ and $\sigma=s-[s]$.

Within the previous framework one can proceed with the finite element analysis. In the following discussion, the parameter $h$ will be assigned to the discretization of the domain $\Omega$ and $H$ to the discretization of the cell $Y$.

In the previous section it is shown that the homogenized coefficients (2.72) are given by

$$
D_{i j k l}(x)=a_{Y}\left(P^{k l}-\chi^{k l}, P^{i j}-\chi^{i j}\right),
$$

where $\chi^{k l}$ is the solution of the following problem:

$$
\text { Find } \chi^{k l} \in V_{y} \text {, such that } a_{Y}\left(X^{k l}-P^{k l}, v\right)=0 \quad \forall v \in V_{y} \text {, }
$$

where the bilinear form $a_{Y}(\cdot, \cdot)$, the set $V_{Y}$, and the tensor $P^{k l}$, are as defined in (2.67), (2.66) and (2.69), respectively. Introducing an abstract finite element discretization satisfying properties (3.1-3.7) for the domain $\boldsymbol{Y}$, a discretized version of problem (3.15) is defined by

$$
\text { Find } X^{H^{k l}} \in V_{Y}^{H} \text {, such that } a_{Y}\left(X^{H^{k l}}-P^{k l}, v^{H}\right)=0 \quad \forall v^{H} \in V_{Y}^{H} \text {. }
$$

The homogenized coefficients (3.14) is then computed by

$$
D_{i j k l}^{H}(x)=a_{y}\left(P^{k l}-\chi^{H^{k l}}, P^{i j}-\chi^{H^{i j}}\right) .
$$

Note that $P^{H^{i j}}=P^{i j}$. Since $V_{Y} \supset V_{y}^{H}, v$ can be chosen as $v=v^{H}$ in (3.15). Subtracting from (3.16) the orthogonality condition in $V_{v}^{H}$ is obtained:

$$
a_{Y}\left(\chi^{k l}-\chi^{H^{k l}}, v^{H}\right)=0 \quad \forall v^{H} \in V_{Y}^{H} .
$$

Using (3.14), (3.17), (3.15) and 3.16) with $v=\chi^{k l}$ and $v^{H}=\chi^{H^{k l}}$, respectively, the difference between the finite element approximation and the exact value of the homogenized coefficients becomes 
160

$$
D_{i j k l}-D_{i j k l}^{H}=a_{Y}\left(\chi^{H^{i j}}-\chi^{i j}, P^{k l}\right) \text {. }
$$

Taking $v=\chi^{i j}$ in (3.15) and $v^{H}=\chi^{H^{i j}}$ in (3.16), and applying the symmetry condition of the bilinear form, one can obtain

$$
a_{Y}\left(\chi^{H^{i j}}, \chi^{H^{k l}}-P^{k l}\right)-a_{Y}\left(\chi^{i j}, \chi^{k l}-P^{k l}\right)=0 .
$$

Similarly, choosing $v^{H}=\chi^{H^{i j}}$ and $v^{H}=\chi^{H^{k l}}$ in (3.18) implies

$$
a_{Y}\left(\chi^{H^{i j}}, \chi^{k l}-\chi^{H^{k l}}\right)-a_{Y}\left(\chi^{i j}-\chi^{H^{i j}}, \chi^{H^{k l}}\right)=0 \text {. }
$$

Adding (3.20) and (3.21) to (3.19) yields

$$
D_{i j k l}^{H}-D_{i j k l}=u_{Y}\left(x^{H^{i j}}-\chi^{i j}, \chi^{H^{k l}}-\chi^{k l}\right) .
$$

This is the error of the finite element approximation of the homogenized elastic coefficients. It is noted that $D_{i j k l}^{H}-D_{i j k l} \geqslant 0$ whenever $(i, j)=(k, l)$ due to property $(2.79)$ of the bilinear form $a_{Y}(\cdot, \cdot)$. Using Schwarz' inequality (2.81) in (3.16), one can obtain

$$
\left|D_{i j k l}^{H}-D_{i j k l}\right| \leqslant\left\{a_{Y}\left(\chi^{H^{i j}}-\chi^{i j}, \chi^{H^{i j}}-\chi^{i j}\right)\right\}^{1 / 2}\left\{a_{Y}\left(\chi^{H^{k l}}-\chi^{k l}, \chi^{H^{k l}}-\chi^{k l}\right)\right\}^{1 / 2} .
$$

Thus the error of the homogenized coefficients is explicitly connected with that of the finite element solution $\chi^{H^{\prime \prime}}$ of problem (3.16). Note that, due to the orthogonality condition (3.18),

$$
a_{Y}\left(\chi^{H^{\prime \prime}}-\chi^{i j}, \chi^{H^{\prime \prime}}-\chi^{i j}\right)=a_{Y}\left(\chi^{H^{\prime \prime}}-\chi^{i j}, v^{H}-\chi^{i j}\right) \quad \forall v^{H} \in V_{Y}^{H} \text {. }
$$

Applying Schwarz' inequality (2.81), it follows from (3.24) that

$$
\left\{a_{Y}\left(\chi^{H^{i j}}-\chi^{i j}, \chi^{H^{i j}}-\chi^{i j}\right)\right\}^{1 / 2} \leqslant\left\{a_{Y}\left(v^{H}-\chi^{i j}, v^{H}-\chi^{i j}\right)\right\}^{1 / 2} \quad \forall v^{H} \in V_{Y}^{H} .
$$

Note that due to (2.79) and (2.80) (see Remarks 2.12-2.20) the bilinear form $a_{Y}(\cdot, \cdot)$ is equivalent to the semi-norm $|\cdot|_{1, Y}$ in $V_{Y}$ and to the norm $\|[\cdot]\|$ in the quotient space $V^{\bullet}$. Thus, for the constant $c_{1} \in \mathbb{R}^{3}$ such that $\left\|\chi^{i j}+c\right\|_{1, v}$ is minimum and for ${ }^{1} \chi^{i j}=\chi^{i j}+c_{1}$, the following relations hold:

$$
\begin{aligned}
a_{Y}\left(v^{H}-\chi^{i j}, v^{H}-\chi^{i j}\right)^{1 / 2} & =a_{Y}\left(v^{H}-{ }^{1} \chi^{i j}, v^{H}-{ }^{1} \chi^{i j}\right)^{1 / 2} \leq \beta\left|v^{H}-{ }^{1} \chi^{i j}\right|_{1, Y} \\
& \leqslant \beta\left\|v^{H}-{ }^{1} \chi^{i j}\right\|_{1, Y} \quad \forall v^{H} \in V_{Y}^{H},
\end{aligned}
$$

where $\beta$ is the same constant in (2.79). Therefore, letting $\chi_{I}^{i j}$ be the finite element interpolation of ${ }^{1} \chi^{i j}$ satisfying (3.11), the following error estimate can be derived for the finite element approximation by taking $\boldsymbol{v}^{H}=\chi_{I}^{i j}$ in the previous equation,

$$
a_{Y}\left(\chi_{I}^{i j}-{ }^{1} \chi^{i j}, \chi_{I}^{i j}-{ }^{1} \chi^{i j}\right)^{1 / 2} \leqslant C H^{\mu}\left\|^{1} \chi^{i j}\right\|_{l, Y}
$$


with $\mu=\min (t-1, l-1)$. Consequently an error estimate for the homogenized elastic constants is obtained as

$$
\left|D_{i j k l}^{H}-D_{i j k l}\right| \leqslant C H^{2 \mu}\left\|^{1} \chi^{i j}\right\|_{l, \Psi}\left\|^{1} \chi^{k l}\right\|_{l, Y}
$$

with $\mu=\min (t-1, l-1)$. If $\chi^{i j} \in\left(H^{2}(₹)\right)^{3}$, then it follows from (3.27) that the energy norm of the approximation error for $\chi_{I}^{H^{i j}}$ has linear convergence rate. It is, however, noted that $\chi^{i j}$ do not belong to $\left(H^{2}(Y)\right)^{3}$ in most of the problems, since material constants in the basic cell need not be smooth, for example, $E_{i j k l} \in L^{\infty}(Y)$ in most of the problems. Thus the convergence rate is, in general, smaller than one.

Similarly the 'residual' stresses are defined in the previous section by (2.74) and (2.75):

$$
\tau_{i j}=a_{Y}\left(\psi, P^{i j}\right),
$$

where $\psi$ is the solution of the problem

Find $\psi \in V_{Y}$, such that $a_{Y}(\psi, v)=f_{Y}(v) \quad \forall v \in V_{Y}$.

Thus, introducing the finite element approximation in the cell domain $\boldsymbol{Z}$, and assuming all the properties for the approximate spaces appearing for the homogenized coefficients still hold, finite element approximations of the residual stresses $\tau_{i j}$ are given by

$$
\tau_{i j}^{H}=a_{Y}\left(\psi^{H}, P^{i j}\right),
$$

where $\psi^{H}$ is the solution of the finite element approximation of (3.30):

Find $\psi^{H} \in V_{Y}^{H}$, such that $a_{Y}\left(\psi^{H}, v^{H}\right)=f_{Y}\left(v^{H}\right) \quad \forall v^{H} \in V_{Y}^{H}$.

Once more, the fact that $V_{v} \supset V_{v}^{H}$ holds yields the orthogonality of the finite element approximation error in $V_{y}^{H}$ such that

$$
a_{Y}\left(\psi-\psi^{H}, v^{H}\right)=0 \quad \forall v^{H} \in V_{Y}^{H} .
$$

Following similar steps for the homogenized elastic constants, the approximation error of the finite element method for the residual stresses is obtained as

$$
\tau_{i j}^{H}-\tau_{i j}=a_{Y}\left(\psi^{H}-\psi, P^{i j}\right),
$$

and then the Schwarz inequality (2.81) implies

$$
\left|\tau_{i j}^{H}-\tau_{i j}\right|=a_{Y}\left(\psi^{H}-\psi, \psi^{H}-\psi\right)^{1 / 2} a_{Y}\left(P^{i j}, P^{i j}\right)^{1 / 2},
$$

where no summation is taken in indices $i$ and $j$. Now the remaining step is to derive an estimate of the quantity $a_{Y}\left(\psi^{H}-\psi, \psi^{H}-\psi\right)^{1 / 2}$. Adding and subtracting $v^{H}$ in the second 
term of $a_{Y}\left(\psi^{H}-\psi, \psi^{H}-\psi\right)$, applying the orthogonality condition (3.33) and Schwarz inequality (2.81), one can obtain

$$
\begin{aligned}
& a_{Y}\left(\psi^{H}-\psi, \psi^{H}-\psi\right) \leqslant a_{Y}\left(\psi^{H}-\psi, v^{H}-\psi\right) \\
& \quad \leqslant a_{Y}\left(\psi^{H}-\psi, \psi^{H}-\psi\right)^{1 / 2} a_{Y}\left(v^{H}-\psi, v^{H}-\psi\right)^{1 / 2} \quad \forall v^{H} \in V_{Y}^{H} .
\end{aligned}
$$

Thus, it follows from (3.36) that

$$
a_{Y}\left(\psi^{H}-\psi, \psi^{H}-\psi\right)^{1 / 2} \leqslant a_{Y}\left(v^{H}-\psi, v^{H}-\psi\right)^{1 / 2} \quad \forall v^{H} \in V_{Y}^{H}
$$

Choosing $c_{1} \in \mathbb{R}^{3}$ that minimizes $\|\boldsymbol{\psi}+c\|_{1, \mathbb{*}}$ and defining ${ }^{1} \boldsymbol{\psi}=\boldsymbol{\psi}+c_{1}$ yields

$$
\begin{aligned}
& a_{Y}\left(\psi^{H}-\psi, \psi^{H}-\psi\right)^{1 / 2}=a_{Y}\left(\psi^{H}-{ }^{1} \psi, \psi^{H}-{ }^{1} \psi\right)^{1 / 2} \leqslant a_{Y}\left(v^{H}-{ }^{1} \psi, v^{H}-{ }^{1} \psi\right)^{1 / 2} \\
& \quad \leqslant \beta\left|v^{H}-{ }^{1} \psi\right|_{1, Y} \leqslant \beta\left\|v^{H}-{ }^{1} \psi\right\|_{1, Y} \quad \forall v^{H} \in V_{Y}^{H},
\end{aligned}
$$

where inequalities in (2.79) are applied to obtain the last two inequalities. It follows from the properties in (3.11) of the interpolation space $V_{y}^{H}$ that there is at least one $v^{H}=v_{I}^{H} \in V_{*}^{H}$ for which the following estimate holds:

$$
\left|\tau_{i j}^{H}-\tau_{i j}\right| \leqslant C H^{\mu}\left\|^{1} \psi\right\|_{l, \boldsymbol{V}} \beta a_{Y}\left(P^{i j}, P^{i j}\right)=C_{0} H^{\mu}\left\|^{1} \psi\right\|_{l, \boldsymbol{Y}},
$$

where $\mu=\min (t-1, l-1)$ and $C_{0}=C \beta a_{Y}\left(P^{i j}, P^{i j}\right)$. Note that the rate of convergence of the discretized problems when $H \rightarrow 0$ depends on the regularity of the respective continuum solutions.

Up to this point error estimations have been introduced for the homogenized coefficients and the 'residual stresses'. Based on these results we can then proceed to obtain the error estimation for the macroscopic problem:

$$
\text { Find } u^{0} \in V_{\Omega}, \text { such that } a\left(u^{0}, v\right)=f(v) \quad \forall v \in V_{\Omega} \text {, }
$$

where $a(\cdot, \cdot)$ and $f(\cdot)$ are as defined in (2.76) and (2.77). It is noted that the homogenized coefficients $D_{i j k l}$ appear in the definition of the bilinear form $a(\cdot, \cdot)$ and that the 'residual' stresses $\tau$ are related to the definition of the linear functional $f(\cdot)$. Since the microscopic cell problems have to be solved prior to the macroscopic homogenized one, finite element approximations of (3.40) are defined using the finite element approximations of the homogenized coefficients and 'residual' stresses:

where

Find $u^{0^{h}} \in V_{\Omega}^{h}$, such that $a^{H}\left(u^{0^{h}}, v^{h}\right)=f^{H}\left(v^{h}\right) \quad \forall v^{h} \in V_{\Omega}^{h}$,

$$
a^{H}(u, v)=\int_{\Omega} D_{i j k l}^{H} \frac{\partial u_{k}}{\partial x_{l}} \frac{\partial v_{i}}{\partial x_{j}} \mathrm{~d} \Omega
$$

and

$$
f^{H}(v)=\int_{\Omega} f_{i} v_{i} \mathrm{~d} \Omega+\int_{\Gamma_{\mathrm{t}}} t_{i} v_{i} \mathrm{~d} \Gamma+\int_{\Omega} \tau_{i j}^{H} \frac{\partial v_{i}}{\partial x_{j}} \mathrm{~d} \Omega .
$$


Since the properties in (2.56) hold for the homogenized coefficients $D_{i j k l}^{H}$, the bilinear form $a^{H}(\cdot, \cdot)$ also has the properties described in (2.82) and (2.83) (see Remarks $(2.12-2.20)$. Thus one may use it as a measure for the error of the solution $u^{0^{h}}$ with respect to $u^{0}$, or, more specifically, the quantity $a^{H}\left(u^{0^{h}}-u^{0}, u^{0^{h}}-u^{0}\right)$ is regarded as the norm for error estimates of the macroscopic problem.

Before proceeding further, it is worthwhile to note that the following quasi-orthogonality property holds in $V_{\Omega}^{h}$ :

where

$$
a\left(u^{0}, v^{h}\right)-a^{H}\left(u^{0^{h}}, v^{h}\right)=\left(f-f^{H}\right)\left(v^{h}\right) \quad \forall v^{h} \in V_{\Omega}^{h},
$$

$$
\left(f-f^{H}\right)\left(\boldsymbol{v}^{h}\right)=\int_{\Omega}\left(\tau_{i j}-\tau_{i j}^{H}\right) \frac{\partial v_{i}^{h}}{\partial x_{j}} \mathrm{~d} \Omega .
$$

Now, turning to the error evaluation, we have

$$
\begin{aligned}
& a^{H}\left(u^{0}-u^{0^{h}}, u^{0}-u^{0^{h}}\right)=a^{H}\left(u^{0}-u^{0^{h}}, u^{0}-v^{h}\right)+a^{H}\left(u^{0}, v^{h}-u^{0^{h}}\right) \\
& -a^{H}\left(u^{0^{h}}, v^{h}-u^{0^{h}}\right) \quad \forall v^{h} \in V_{\Omega}^{h} .
\end{aligned}
$$

Applying (3.44) implies

where

$$
\begin{aligned}
& a^{H}\left(u^{0}-u^{0^{h}}, u^{0}-u^{0^{h}}\right)=a^{H}\left(u^{0}-u^{0^{h}}, u^{0}-v^{h}\right)+\left(a^{H}-a\right)\left(u^{0}, v^{h}-u^{0^{h}}\right) \\
& \quad+\left(f-f^{H}\right)\left(v^{h}-u^{0^{h}}\right) \quad \forall v^{h} \in V_{\Omega}^{h},
\end{aligned}
$$

$$
\left(a^{H}-a\right)(u, v)=\int_{\Omega}\left(D_{i j k l}^{H}-D_{i j k l}\right) \frac{\partial u_{k}}{\partial x_{l}} \frac{\partial v_{i}}{\partial x_{j}} \mathrm{~d} \Omega \text {. }
$$

For the microscopic cell problems, it was obtained that

$$
\begin{aligned}
& \left|D_{i j k m}^{H}-D_{i j k m}\right| \leqslant C H^{2 \mu}\left\|^{1} \chi^{i j}\right\|_{l, \xi}\left\|^{1} \chi^{k m}\right\|_{l, v}, \\
& \left|\tau_{i j}^{H}-\tau_{i j}\right| \leqslant C_{0} H^{\mu}\|\|^{1} \Psi \|_{l, Y},
\end{aligned}
$$

where these inequalities hold at a specific point $x$ of the domain $\Omega$. Assuming that the homogenized coefficients and the 'residual' stresses are in $\left(L_{\infty}(\Omega)\right)^{3}$, a bound for the above quantities in the whole domain $\boldsymbol{\Omega}$ may be found as

$$
\begin{aligned}
& \left|D_{i j k m}^{H}-D_{i j k m}\right| \leqslant C H^{2 \mu}\left\|^{1} \chi^{i j}\right\|_{l, \xi}\left\|^{1} \chi^{k m}\right\|_{l, \Psi} \text { in } \Omega, \\
& \left|\tau_{i j}^{H}-\tau_{i j}\right| \leqslant C_{0} H^{\mu}\left\|^{1} \Psi\right\|_{l, \Psi} \text { in } \Omega .
\end{aligned}
$$

Applying inequality (3.51) in (3.48), one obtains 


$$
\begin{aligned}
& \left(a^{H}-a\right)\left(u^{0}, v^{h}-u^{0^{h}}\right)=\int_{\Omega}\left(D_{i j k l}^{H}-D_{i j k l}\right) \frac{\partial u_{k}^{0}}{\partial x_{l}} \frac{\partial\left(v_{i}^{h}-u_{i}^{0^{h}}\right)}{\partial x_{j}} \mathrm{~d} \Omega \\
& \quad \leqslant \int_{\Omega} C H^{2 \mu} 9^{3 / 2} \max _{i j k m}\left(\left\|x^{1 j}\right\|_{l, v}\left\|^{1} x^{k m}\right\|_{l, Y}\right)\left|\frac{\partial u_{i}^{0}}{\partial x_{j}} \frac{\partial\left(v_{i}^{h}-u_{i}^{0^{h}}\right)}{\partial x_{j}}\right| \mathrm{d} \Omega \\
& \quad \leqslant 27 C H^{2 \mu} \max _{i j k m}\left(\left\|^{1} \chi^{i j}\right\|_{l, v}\left\|^{1} x^{k m}\right\|_{l, Y}\right)\left|u^{0}\right|_{1, \Omega}\left|v^{h}-u^{0^{h}}\right|_{1, \Omega} \\
& \quad \leqslant 27 C H^{2 \mu} \max _{i j k m}\left(\left\|^{1} x^{i j}\right\|_{l, v}\left\|^{1} x^{k m}\right\|_{l, Y}\right)\left\|u^{0}\right\|_{1, \Omega}\left\|v^{h}-u^{0^{h}}\right\|_{1, \Omega} \\
& \quad=C(H)\left\|u^{0}\right\|_{1, \Omega}\left\|v^{h}-u^{0^{h}}\right\|_{1, \Omega},
\end{aligned}
$$

where

$$
\left.C(H)=2 i C H i^{\prime} \max _{i j k m}\left\|^{\prime} x^{\prime i}\right\|_{l, y}\left\|^{1} \chi^{k m}\right\|_{l, Y}\right) \text {. }
$$

Similarly, applying (3.50) in (3.45), one obtains

$$
\begin{aligned}
& \left(f-f^{H}\right)\left(v^{h}-u^{0^{h}}\right)=\int_{\Omega}\left(\tau_{i j}-\tau_{i j}^{H}\right) \frac{\partial\left(v_{i}^{h}-u_{i}^{0^{h}}\right)}{\partial x_{j}} \mathrm{~d} \Omega \\
& \quad \leqslant \int_{\Omega} C_{0} H^{\mu}\left\|^{1} \Psi\right\|_{l, v} \sum_{i j}\left|\frac{\partial\left(v_{i}^{h}-u_{i}^{0^{h}}\right)}{\partial x_{j}}\right| \mathrm{d} \Omega \\
& \quad \leqslant C_{0} H^{\mu}\left\|^{1} \Psi\right\|_{l, v}\left\|\boldsymbol{v}^{h}-u^{0^{h}}\right\|_{1, \Omega}=C_{0}(H)\left\|\boldsymbol{v}^{h}-u^{0^{h}}\right\|_{1, \Omega},
\end{aligned}
$$

where

$$
C_{0}(H)=H^{\mu}\left\|^{1} \Psi\right\|_{1, Y} .
$$

The substitution of (3.53) and (3.55) in (3.47) and the use of the Schwarz inequality (2.83) yields

$$
\begin{aligned}
& a^{H}\left(u^{0}-u^{0^{h}}, u^{0}-u^{0^{h}}\right) \leqslant a^{H}\left(u^{0}-u^{0^{h}}, u^{0}-u^{0^{h}}\right)^{1 / 2} a^{H}\left(u^{0}-v^{h}, u^{0}-v^{h}\right)^{1 / 2} \\
& +C(H)\left\|u^{0}\right\|_{1, \Omega}\left\|v^{h}-u^{0^{h}}\right\|_{1, \Omega}+C_{0}(H)\left\|v^{h}-u^{0^{h}}\right\|_{1, \Omega} \quad \forall v^{h} \in V_{\Omega}^{h}
\end{aligned}
$$

Noting that the bilinear form is equivalent to norm $\|\cdot\|_{1, \Omega}$ in $V_{\Omega}$, one can obtain

$$
\begin{aligned}
& \alpha^{2}\left\|u^{0}-u^{0^{h}}\right\|_{1 ; \Omega}^{2} \leqslant \beta^{2}\left\|u^{0}-u^{0^{h}}\right\|_{1, \Omega}\left\|u^{0}-v^{h}\right\|_{1, \Omega} \\
& \quad+\left(C(H)\left\|u^{0}\right\|_{1, \Omega}+C_{0}(H)\right)\left\|v^{h}-u^{0^{h}}\right\|_{1, \Omega} \quad \forall v^{h} \in V_{\Omega}^{h},
\end{aligned}
$$

where $\alpha$ and $\beta$ are the same constants as in (2.82). Now, the following inequality is obtained adding and subtracting $u^{0}$ in the second term of the right-hand side of (3.58), applying the triangle inequality, and then using Young's inequality to all products of terms: 


$$
\begin{gathered}
\alpha^{2}\left\|u^{0}-u^{0^{h}}\right\|_{1, \Omega}^{2} \leqslant\left(\beta^{2} e^{2}+\frac{1}{4 d^{2}}\right)\left\|u^{0}-u^{0^{h}}\right\|_{1, \Omega}^{2}+\left(\frac{\beta^{2}}{4 e^{2}}+\frac{1}{4 b^{2}}\right)\left\|u^{0}-v^{h}\right\|_{1, \Omega}^{2} \\
+\left(b^{2}+d^{2}\right)\left(C(H)\left\|u^{0}\right\|_{1, \Omega}+C_{0}(H)\right)^{2} \quad \forall v^{h} \in V_{\Omega}^{h} \forall e, b, d>0 .
\end{gathered}
$$

Choosing $e$ and $d$ so that

$$
A=\alpha^{2}-\beta^{2} e^{2}-\frac{1}{4 d^{2}}>0
$$

and defining

and

$$
A_{1}=\left(\frac{\beta^{2}}{4 e^{2}}+\frac{1}{4 b^{2}}\right) \frac{1}{A}
$$

$$
A_{2}=\left(b^{2}+d^{2}\right) \frac{1}{A}
$$

one obtains

$$
\left\|u^{0}-u^{0^{h}}\right\|_{1, \Omega}^{2} \leqslant A_{1}\left\|u^{0}-v^{h}\right\|_{1, \Omega}^{2}+A_{2}\left(C(H)\left\|u^{0}\right\|_{1, \Omega}+C_{0}(H)\right)^{2} \quad \forall v^{h} \in V_{\Omega}^{h} .
$$

Finally, and due to the properties of the interpolation space $V_{\Omega}^{h}$, there exists a $u_{l}^{0}$ in $V_{\Omega}^{h}$ for which the following estimate holds:

$$
\left\|u^{0}-u^{0^{h}}\right\|_{1, \Omega}^{2} \leqslant A_{1} D h^{2 \sigma}\left\|u^{0}\right\|_{n, \Omega}^{2}+A_{2}\left(C(H)\left\|u^{0}\right\|_{1, \Omega}+C_{0}(H)\right)^{2},
$$

where $\sigma=\min (t-1, n-1), n>1$, and $h$ is a geometric parameter associated to the size of the finite elements in the discretization of the macroscopic domain $\boldsymbol{\Omega}$.

It is noted that the above estimates are obtained under the assumptions that both domains $\boldsymbol{\Omega}$ and $\boldsymbol{Y}$ are polygonal and that the integration is performed exactly. If the domain is not polygonal and the finite element discretization cannot cover exactly the original domains, then the space of functions generated by the finite element discretization $V^{h}$ is no longer a subspace of the initial space $V$. Similarly, if the integration scheme is not exact, integration errors must be additionally considered. Details of nonpolygonal domains and numerical integration errors can be found in, for example, $[17,19]$.

The previous a priori error estimations (3.28), (3.39) and (3.64) provide the convergence rate of the finite element approximations to the continuous solutions when the mesh size of the discretizations converges towards zero, i.e., when $h \rightarrow 0$. As can easily be seen, this convergence rate, the power of the parameter $h$, depends on the regularity of these solutions. Thus, if somehow one can predict their regularity, the convergence rate indicates how much refinement would be necessary to significantly improve the finite element approximations. This is illustrated in the following example. 
EXAMPLE 3.1 (Convergence test for regular meshes). Let us examine the above error estimates for a particular example problem in which both the macroscopic domain $\Omega$ and the microscopic cell $\boldsymbol{Z}$ are rectangles in $\mathbb{R}^{2}$ for simplicity. Both domains are discretized using QUAD4 elements, i.e., the isoparametric four node element, and the macroscopic displacement $\boldsymbol{u}^{0}$ and the characteristics of deformation $\boldsymbol{\chi}^{i j}$, for each $i j$, are interpolated by bilinear polynomials in the parametric space. The rates of convergence are then examined for the macroscopic displacement $u^{0}$ and the homogenized coefficients $D_{i j k l}$.

The microscopic square cell structure is defined by two isotropic materials as shown in Fig. 3.1. Since the convergence of the finite element approximation is the only concern, the materials of the composite are artificially chosen and the microstructure is set uniform throughout the macroscopic domain $\Omega$. Then, the homogenized elastic constants are computed by using $4 \times 4,6 \times 6,8 \times 8,10 \times 10$ and $12 \times 12$ meshes. Similarly, the macroscopic rectangular structure $\Omega$ is discretized by $4 \times 4,6 \times 6,8 \times 8,10 \times 10$ and $12 \times 12$, as shown in Fig. 3.2.

For the microscopic base cell introduced, the solutions $\chi^{i j}$ of problem (3.15) are at most in $H^{1.5-\delta}(Y)$ since $E_{i j k l}$ are in $H^{0.5-\delta}(Y)$, where $\delta$ is an arbitrary positive number. This claim may be justified by the following considerations:

(a) The step function $H(x)(H(x)=1$ for $x>0$ and $H(x)=0$ for $x<0)$ is in $H^{0.5-\delta}(\mathbb{R})$ (see [17]). Applying the concept of the tensor product, the function $H(x, y)=H(x) H(y)$ in $\mathbb{R}^{2}$ may be regarded as a function in $H^{0.5-\delta}\left(\mathbb{R}^{2}\right)$. Since $E_{i j k l}$ can be constructed by linear combinations of appropriate constants and the function $H(x, y), E_{i j k l}$ are in $H^{0.5-\delta}(Y)$.

(b) The strong form of the microscopic base cell problem (3.15) involves the set of differential equations

$$
-\frac{\partial}{\partial y_{j}}\left(E_{i j p m} \frac{\partial \chi_{p}^{k l}}{\partial y_{m}}\right)=-\frac{\partial}{\partial y_{j}} E_{i j k l} \text { in } \boldsymbol{Y} .
$$

This yields at most that

$$
E_{i j p m} \frac{\partial \chi_{p}^{k l}}{\partial y_{m}} \in H^{0.5-\delta}(\eta) .
$$

Since $E_{i j p m} \in H^{0.5-\delta}(Y), \chi_{p}^{k l}$ can be at most in $H^{1.5-\delta}(Y)$.

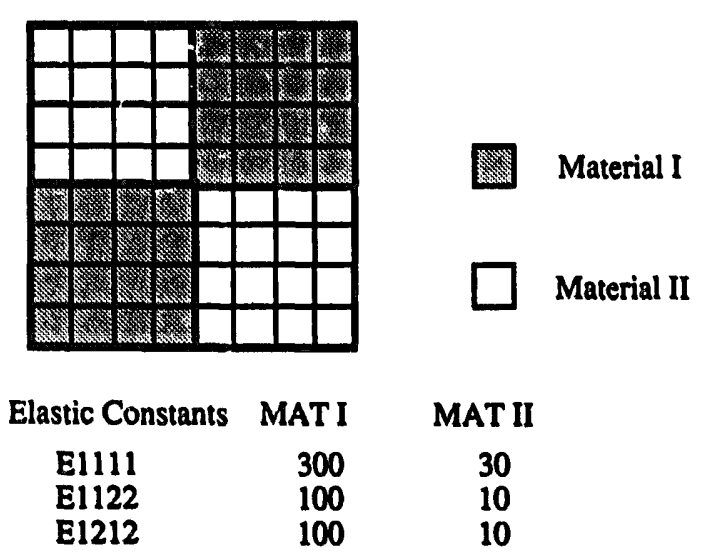

Fig. 3.1. The base cell structure for the convergence test. 

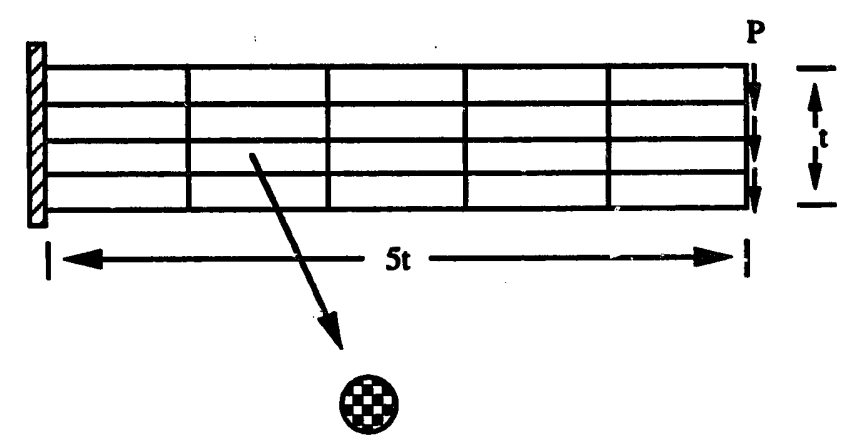

Fig. 3.2. Global structure and its finite element discretization.

Thus, if the error estimate (3.28) is applied to the present problem using the four node bilinear isoparametric finite element QUAD4 for plane problems, $\mu$ is expected to be $0.5-\delta$, since $\mu=\min (2-1,1.5-\delta-1)$ because of $t=2$ for this choice of finite elements. Therefore, the rate of convergence of the homogenized constants $D_{i j k l}^{H}$ by the finite element approximation is $1-2 \delta$, where $\delta$ is an arbitrary positive constant. In practice, $\delta$ can be regarded to be zero.

In order to present the numerical results it is convenient to note that

$$
\frac{1}{D_{i j k l}^{H}}-\frac{1}{D_{i j k l}}=\frac{D_{i j k l}-D_{i j k l}^{H}}{D_{i j k l} D_{i j k l}^{H}} \approx C H^{1-2 \delta},
$$

where $C$ is an appropriate positive constant. If the relations $\left(H^{1-2 \delta}, 1 / D_{i j k l}^{H}\right)$ for different values of $H$ are plotted, they should be liniear. Indeed, as shown in Fig. 3.3, all the independent combination of indices- $(i, j, k, l)=(1,1,1,1),(i, j, k, l)=(1,1,2,2)$ and $(i, j$, $k, l)=(1,2,1,2)$-form linear lines. Furthermore, these lines predict the 'exact' homogenized elastic constants $D_{1111}, D_{1122}$ and $D_{1212}$ :

$$
D_{1111}=7.71724, \quad D_{1122}=3.15080 \text { and } D_{1212}=3.27182 \text {. }
$$

If these values are used as the 'exact' homogenized elastic constants, $D_{1111}$ is calculated with $15.6 \%$ error using the $4 \times 4$ mesh $(H=0.125)$, while it is calculated with $4.6 \%$ error using the $12 \times 12$ mesh $(H=0.0417)$. Applying 9 times more finite elements, only 3.4 times better estimation can be obtained if uniform refinement is considered. As shown in Fig. 3.3, the rate of convergence is slow, and furthermore, there is a relatively large amount of finite element approximation error even in very refined cases.

On the other hand, if the macroscopic problem (3.40) is considered, its solution $u^{0}$ is expected to be at least in $\boldsymbol{H}^{2}(\Omega)$, since only a constant traction $t$ is applied along the free end of the beam-like rectangular structure $\Omega$, and each component of the homogenized elasticity tensor is a constant function of $x$ in $\Omega$. Thus, the error estimate (3.64) yields the unit rate of convergence, i.e., the error in the $H^{1}$-norm, which is equivalent to the energy norm, is proportional to $h^{1}$, where $h$ is the representative element size in the macroscopic structure. 


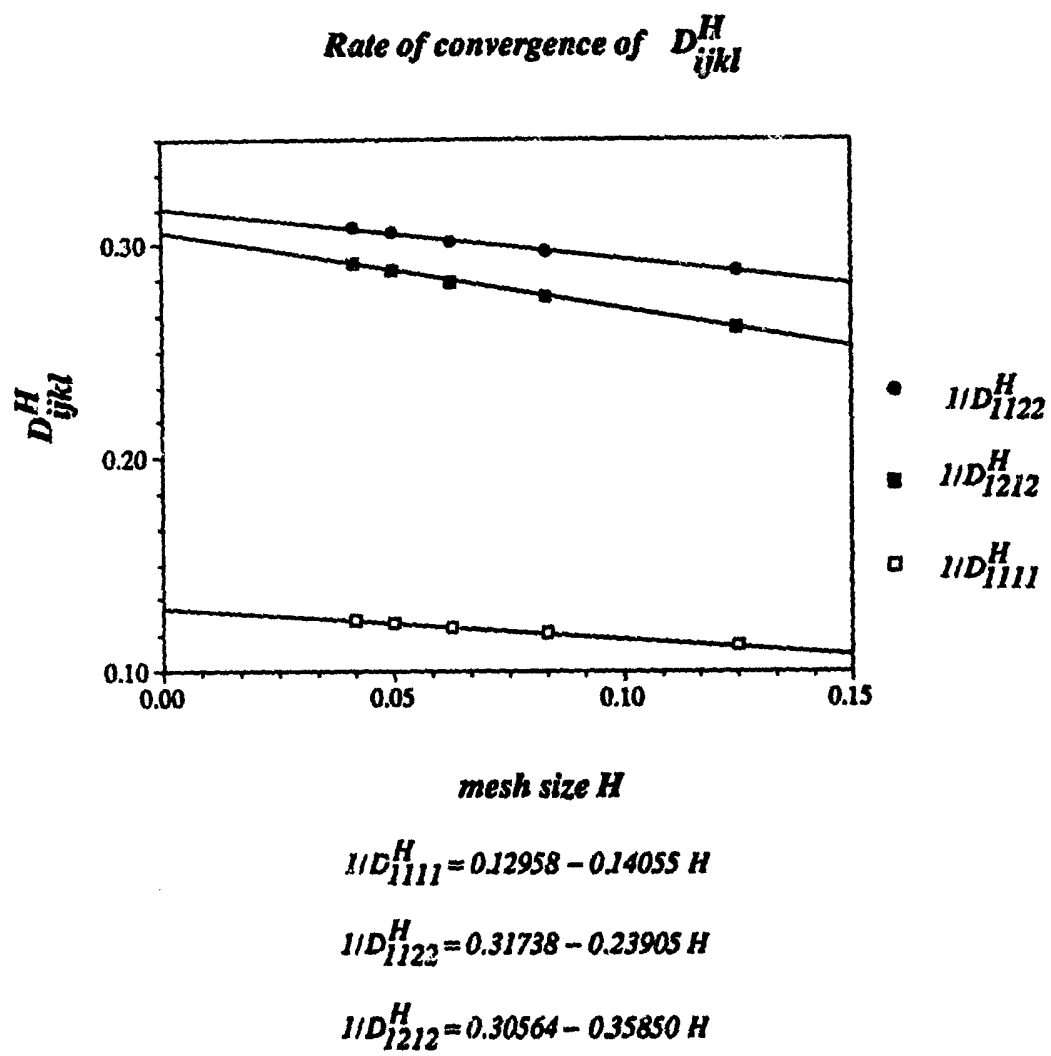

Fig. 3.3. Convergence of the homogenized elastic constants.

In order to present the results, note that because the bilinear form $a^{H}(\cdot, \cdot)$ is symmetric, the following identity holds:

$$
a^{H}\left(u^{0}, u^{0}\right)-a^{H}\left(u^{0^{h}}, u^{0^{h}}\right)=a^{H}\left(u^{0}-u^{0^{h}}, u^{0}-u^{0^{h}}\right)+2 a^{H}\left(u^{0}-u^{0^{h}}, u^{0^{h}}\right) .
$$

It then follows from the orthogonality of the finite element approximation error that

$$
a^{H}\left(u^{0}, u^{0}\right)-a^{H}\left(u^{0^{h}}, u^{0^{h}}\right)=a^{H}\left(u^{0}-u^{0^{h}}, u^{0}-u^{0^{h}}\right) .
$$

Taking the reciprocal relation implies

$$
\frac{1}{a^{H}\left(u^{0^{h}}, u^{0^{h}}\right)}-\frac{1}{a^{H}\left(u^{0}, u^{0}\right)}=\frac{a^{H}\left(u^{0}, u^{0}\right)-a^{H}\left(u^{0^{h}}, u^{0^{h}}\right)}{a^{H}\left(u^{0^{h}}, u^{0^{h}}\right) a^{H}\left(u^{0}, u^{0}\right)}
$$

Note that the energy norm defined by the bilinear form $a^{H}(\cdot, \cdot)$ is equivalent to the $H^{1}(\Omega)$ norm. Then applying the error estimate (3.64) yields

$$
\frac{1}{a^{H}\left(u^{0^{h}}, u^{0^{h}}\right)}-\frac{1}{a^{H}\left(u^{0}, u^{0}\right)} \approx C_{1} h^{2}+C_{2} H^{1-2 \delta},
$$


where $C_{1}$ and $C_{2}$ are appropriate positive constants. This means that if the relations $\left(h^{2}\right.$, $\left.1 / a^{H}\left(u^{0^{h}}, u^{0^{h}}\right)\right)$ are plotted for different $h$, they should be linear. Indeed, Fig. 3.4 shows this fact. Furthermore, the value of $a^{H}\left(u^{0}, u^{0}\right)$ can be estimated using the linear relation within a constant $C_{2} H^{1-2 \delta}$.

Since the $L^{\infty}(\Omega)$ norm of the displacement $u^{0}$ behaves similarly to the strain energy, the finite element approximation of the beam free end displacement converges to the exact one with the same rate of convergence, $h^{2}$, to the strain energy. Thus, the relations $\left(h^{2}, 1 / d\right)$, where $d=\left.u^{0^{h}}\right|_{\text {at free end }}$, for different $h$ is linear for each choice of $H$, see Fig. 3.4. In Fig. 3.4, the quantities with superimposed ' $*$ ' are the macroscopic response obtained by using the homogenized elastic constants computed by a coarse finite element mesh, say a $4 \times 4$ mesh, for the microscopic problem. It is clear that poor finite element approximation of the homogenized elastic constants implies stiff macroscopic response.

This example illustrates that as a consequence of the convergence properties of the finite element approximations of the microscopic and macroscopic problems, uniform mesh refinements yield a fairly accurate estimation of the 'exact' solutions by extrapolating from their approximations.

\section{Rate of Convergence (Macroscopic)}

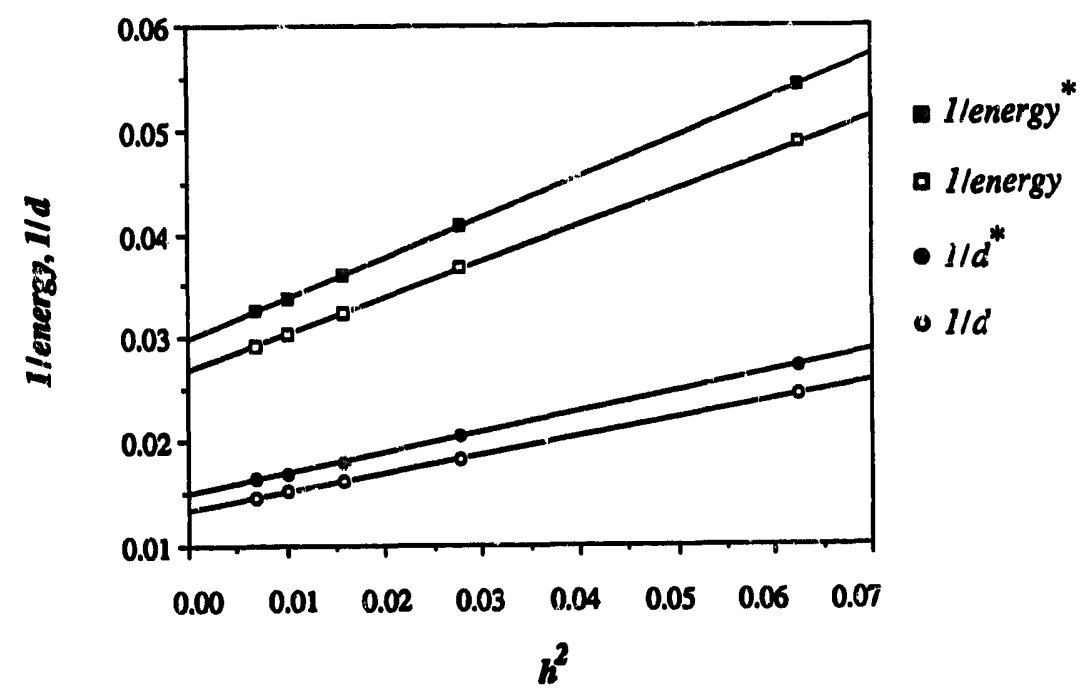

$$
\begin{aligned}
& \text { llenergy }{ }^{*}=2.0951 e^{-2}+0.39116 h^{2} \\
& \text { Ilenergy }=2.6815 e^{-2}+0.3517 h^{2} \\
& 1 / d^{*}=1.4982 e^{-2}+0.1953 h^{2} \\
& I / d=1.3413 e^{-2}+0.1757 h^{2}
\end{aligned}
$$

Fig. 3.4. Convergence of the strain energy and the deflection of the global structure. 


\section{Implementation of the homogenization method: PREMAT and POSTMAT}

In this section the finite element implementation of the homogenization method is presented. This implementation is formed by two separated blocks. The first, PREMAT, generates the material properties for a general structure. The second, POSTMAT, computes localized information (displacements, stresses and strains) after a finite element procedure has been used to solve the general structure.

For simplicity, let us apply QUAD4 and HEXA8 elements to discretize the cell domain $\mathbf{Y}$ for two-dimensional and three-dimensional finite element analyses of the microscopic cell problem. To this end, after constructing the finite element approximation $V^{h}$ of the space $V$ using these bilinear and trilinear elements, discrete problems (3.15) and (3.30) will be solved to obtain the finite element approximations of the homogenized elastic constants and the 'residual' stresses for the homogenized macroscopic problem (3.40). Applying the usual implementation procedure of the finite element method, an arbitrary function $v^{H}$ in the space $V_{\psi}^{H}$ is interpolated by using the shape functions $N_{\alpha}(\xi)$ :

$$
v^{H}(y)=\sum_{\alpha=1}^{N_{e}} v_{\alpha}^{H} N_{\alpha}(\xi), \quad N_{e}=4 \text { or } 8,
$$

while the geometry of an element $Y_{e}$ in $Z$ is also interpolated using the same shape functions $N_{\alpha}$ :

$$
y=\sum_{\alpha=1}^{N_{e}} y_{\alpha} N_{\alpha}(\xi), \quad N_{e}=4 \text { or } 8,
$$

where the shape functions $N_{\alpha}(\xi)$ are defined by

$$
N_{\alpha}(\xi)=\left\{\begin{array}{l}
\frac{1}{4}\left(1+\xi_{1 \alpha} \xi_{1}\right)\left(1+\xi_{2 \alpha} \xi_{2}\right), \\
\frac{1}{8}\left(1+\xi_{1 \alpha} \xi_{1}\right)\left(1+\xi_{2 \alpha} \xi_{2}\right)\left(1+\xi_{3 \alpha} \xi_{3}\right),
\end{array}\right.
$$

and $\left\{\left(\xi_{1 \alpha}, \xi_{2 \alpha}\right)\right\}$ and $\left\{\left(\xi_{1 \alpha}, \xi_{2 \alpha}, \xi_{3 \alpha}\right)\right\}, \alpha=1, \ldots, N_{e}$, are the coordinates of the corresponding nodes $\alpha=1, \ldots, N_{e}$ in the master element $\Omega_{M}$ of the QUAD4 and HEXA8 elements, respectively. Introducing these into (3.16) the discretized problem is obtained as

$$
\text { Find } X^{H^{k l}} \in V_{v}^{H} \text {, such that } \sum_{e=1}^{E} a_{Y e}\left(X H^{k l}-P^{k l}, v^{H}\right)=0 \quad \forall v^{H} \text {, }
$$

where

$$
a_{Y e}(u, v)=\frac{1}{|Y|} \int_{\Psi_{e}} E_{i j k l} \frac{\partial u_{k}}{\partial y_{l}} \frac{\partial v_{i}}{\partial y_{j}} \mathrm{~d} Y, \quad u, v \in V_{\boldsymbol{v}}^{H}
$$

Since the shape functions are the same for all the cases of $x^{H^{k l}}, k, l=1,2$ and 3 , the stiffness matrix to different indices of $k$ and $l$ is also the same for all of $k$ and $l$. Thus, once the stiffness matrix $K$ is formed and decomposed in $L U$ form, where $L$ and $U$ are the lower and upper 
triangular matrices of $K$ for $k=l=1$ from the bilinear form $a_{Y}(\cdot, \cdot)$, they are applicable for other $k$ and $l$. This means that solving discrete problems to determine the characteristic deformations $\boldsymbol{X}^{H^{k l}}$ for six independent ones, is not so expensive in the sense that the right-hand sides for the six independent cases can be formed and reduced with less intensive computation. Similarly, finite element approximations of the 'residual' stresses $\tau_{i j}^{H}$ are obtained by solving (3.32):

where

$$
\text { Find } \psi^{H} \in V_{Y}^{H} \text {, such that } \sum_{e=1}^{E} a_{Y e}\left(\psi^{H}, v^{H}\right)=\sum_{e=1}^{E} f_{Y e}\left(v^{H}\right) \quad \forall v^{H} \in V_{Y}^{\Psi},
$$

$$
f_{Y e}(v)=\frac{1}{|Y|} \int_{\partial \Psi_{e}} p_{i} v_{i} \mathrm{~d} S
$$

and $\partial \Psi_{e}$ is the surface of an element $\Psi_{e}$ that intersects to the boundary $S$ of the holes of the basic cell $\Psi$. Here, $\psi^{H}$ are interpolated by the shape functions $N_{\alpha}$ :

$$
\psi^{H}=\sum_{\alpha=1}^{N_{e}} \psi_{\alpha}^{H} N_{\alpha}(\xi) \text { in } \Psi_{e} .
$$

Once $\chi^{H^{k l}}$ and $\psi^{H}$ are obtained, using (3.17) and (3.31) one can compute the finite element approximations of the homogenized elastic constants and the 'residual' stresses for the macroscopic homogenized problem.

The program PREMAT has been developed to compute material constants of generalized materials for finite element analysis based on the homogenization method described in above. The basic structure of PREMAT is described as follows:

read structuretype

if structuretype.eq.solid

then

read materialtype

if materialtype.eq.anisotropic

then

read $E 1, \nu 12, \nu 13, \nu 123, \nu 131, \nu 112$, and other engineering constants call dmatrix

else

if materialtype.eq.orthotropic

then

read $E 1, \nu 12, \nu 13$, and other engineering constants

read Euler's angles of the material axes

call dmatrix

call rotation

else

if materialtype.eq.isoțropic

then

read $E$ and $\nu$

call dmatrix 


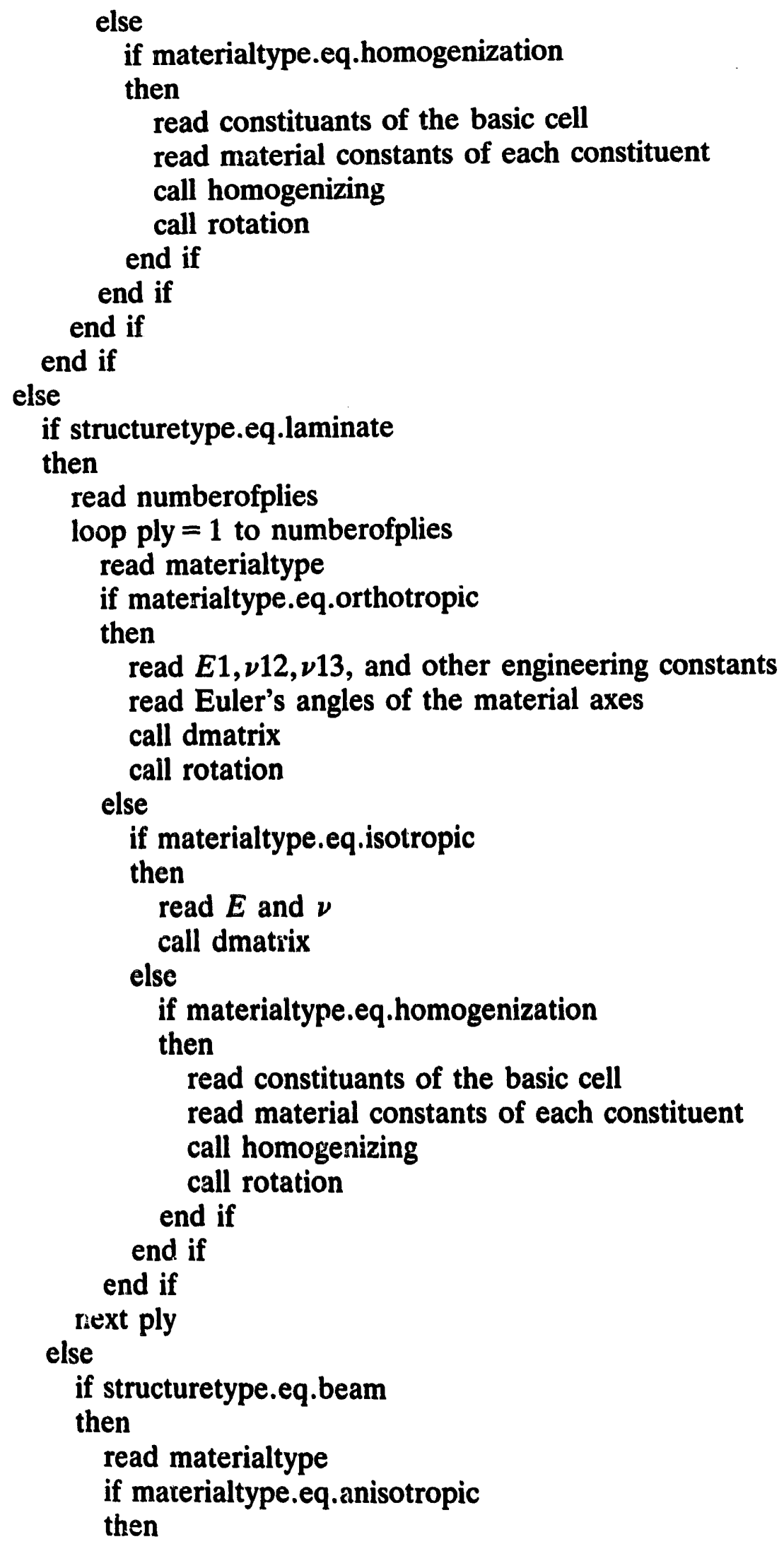


read $E 1, \nu 12, \nu 112$, and other engineering constants call dmatrix else

if materialtype.eq.orthotropic

then

read $E 1, \nu 12$, and other engineering constants

read Euler's angle of the material axes

call dmatrix

call rotation

else

if materialtype.transverseisotropic

then

read $E 1, E 2, \nu 12$, and theta

call dmatrix

call rotation

else

if materialtype.eq.isotropic

then

read $E$ and $\nu$

call dmatrix

else

if materialtype.eq.homogenization

then

read constituants of the basic cell

read material constants of each constituent

call homogenizing

call rotation

end if

end if

end if

end if

end if

end if

end if.

Here subroutine dmatrix forms the so-called $D$ matrix, the matrix of the material constants, in the finite element analysis, while subroutine rotation rotates $D$ to the coordinate system in which the element stiffness matrices are computed. If the material type is standard, so that engineering constants such as Young's moduli, Poisson's ratios and shear moduli are already known by, for example, tests in a laboratory, it is not necessary to apply the homogenization method to determine the elastic constants forming the $D$ matrix.

In the homogenization method, the reduced integration method with hourglass control is applied to form the element stiffness matrices from the bilinear form $a_{Y_{e}}(\cdot, \cdot)$ in order to reduce computing time in three-dimensional problems. Details of such a method can be found in, for example, [20]. Here only a brief description of the method is given. Thus, for the 
HEXA8 element, there exists the following isoparametric geometry relation between an arbitrary finite element ( $x$-coordinates) and the master element ( $\xi$-coordinates):

$$
\begin{aligned}
{\left[\begin{array}{l}
x_{1} \\
x_{2} \\
x_{3}
\end{array}\right]=} & {\left[\begin{array}{lll}
l_{1} \cdot x_{1} & l_{2} \cdot x_{1} & l_{3} \cdot x_{1} \\
l_{1} \cdot x_{2} & l_{2} \cdot x_{2} & l_{3} \cdot x_{2} \\
l_{1} \cdot x_{3} & l_{2} \cdot x_{3} & l_{3} \cdot x_{3}
\end{array}\right]\left[\begin{array}{l}
\xi_{1} \\
\xi_{2} \\
\xi_{3}
\end{array}\right] } \\
& +\left[\begin{array}{ll}
c \cdot x_{1}+h_{1} \cdot x_{1} \xi_{2} \xi_{3}+h_{2} \cdot x_{1} \xi_{3} \xi_{1}+h_{3} \cdot x_{1} \xi_{1} \xi_{2}+h_{4} \cdot x_{1} \xi_{1} \xi_{2} \xi_{3} \\
c \cdot x_{2}+h_{1} \cdot x_{2} \xi_{2} \xi_{3}+h_{2} \cdot x_{2} \xi_{3} \xi_{1}+h_{3} \cdot x_{2} \xi_{1} \xi_{2}+h_{4} \cdot x_{2} \xi_{1} \xi_{2} \xi_{3} \\
c \cdot x_{3}+h_{1} \cdot x_{3} \xi_{2} \xi_{3}+h_{2} \cdot x_{3} \xi_{3} \xi_{1}+h_{3} \cdot x_{3} \xi_{1} \xi_{2}+h_{4} \cdot x_{3} \xi_{1} \xi_{2} \xi_{3}
\end{array}\right],
\end{aligned}
$$

where

$$
\begin{array}{ll}
l_{1}^{\mathrm{t}}=\frac{1}{8}\{-1,1,1,-1,-1,1,1,-1\}, & l_{2}^{\mathrm{t}}=\frac{1}{8}\{-1,-1,1,1,-1,-1,1,1\}, \\
l_{3}^{\mathrm{t}}=\frac{1}{8}\{-1,-1,-1,-1,1,1,1,1\}, & c^{\mathrm{t}}=\frac{1}{8}\{1,1,1,1,1,1,1,1\}, \\
h_{1}^{\mathrm{t}}=\frac{1}{8}\{1,1,-1,-1,-1,-1,1,1\}, & h_{2}^{\mathrm{t}}=\frac{1}{8}\{1,-1,1,-1,-1,1,1,-1\}, \\
h_{3}^{\mathrm{t}}=\frac{1}{8}\{1,-1,1,-1,1,-1,1,-1\}, \quad h_{4}^{\mathrm{t}}=\frac{1}{8}\{-1,1,-1,1,1,-1,1,-1\}, \\
x_{1}^{\mathrm{t}}=\left\{x_{11}, x_{12}, x_{13}, x_{14}, x_{15}, x_{16}, x_{17}, x_{18}\right\}, \\
x_{2}^{\mathrm{t}}=\left\{x_{21}, x_{22}, x_{23}, x_{24}, x_{25}, x_{26}, x_{27}, x_{28}\right\}, \\
x_{3}^{\mathrm{t}}=\left\{x_{31}, x_{32}, x_{33}, x_{34}, x_{35}, x_{36}, x_{37}, x_{38}\right\} .
\end{array}
$$

The inverse relation can be obtained as

$$
\begin{aligned}
{\left[\begin{array}{l}
\xi_{1} \\
\xi_{2} \\
\xi_{3}
\end{array}\right]=} & {\left[\begin{array}{lll}
l_{1} \cdot x_{1} & l_{2} \cdot x_{1} & l_{3} \cdot x_{1} \\
l_{1} \cdot x_{2} & l_{2} \cdot x_{2} & l_{3} \cdot x_{2} \\
l_{1} \cdot x_{3} & l_{2} \cdot x_{3} & l_{3} \cdot x_{3}
\end{array}\right]^{-1}\left[\begin{array}{l}
x_{1} \\
x_{2} \\
x_{3}
\end{array}\right] } \\
& -\left[\begin{array}{lll}
l_{1} \cdot x_{1} & l_{2} \cdot x_{1} & l_{3} \cdot x_{1} \\
l_{1} \cdot x_{2} & l_{2} \cdot x_{2} & l_{3} \cdot x_{2} \\
l_{1} \cdot x_{3} & l_{2} \cdot x_{3} & l_{3} \cdot x_{3}
\end{array}\right] \\
& \times\left[\begin{array}{ll}
c \cdot x_{1}+h_{1} \cdot x_{1} \xi_{2} \xi_{3}+h_{2} \cdot x_{1} \xi_{3} \xi_{1}+h_{3} \cdot x_{1} \xi_{1} \xi_{2}+h_{4} \cdot x_{1} \xi_{1} \xi_{2} \xi_{3} \\
c \cdot x_{2}+h_{1} \cdot x_{2} \xi_{2} \xi_{3}+h_{2} \cdot x_{2} \xi_{3} \xi_{1}+h_{3} \cdot x_{2} \xi_{1} \xi_{2}+h_{4} \cdot x_{2} \xi_{1} \xi_{2} \xi_{3} \\
c \cdot x_{3}+h_{1} \cdot x_{3} \xi_{2} \xi_{3}+h_{2} \cdot x_{3} \xi_{3} \xi_{1}+h_{3} \cdot x_{3} \xi_{1} \xi_{2}+h_{4} \cdot x_{3} \xi_{1} \xi_{2} \xi_{3}
\end{array}\right]
\end{aligned}
$$

Since a similar expression to (4.9) can be obtained for a 'displacement' vector $v$ :

$$
\begin{aligned}
{\left[\begin{array}{l}
v_{1} \\
v_{2} \\
v_{3}
\end{array}\right]=} & {\left[\begin{array}{lll}
l_{1} \cdot v_{1} & l_{2} \cdot v_{1} & l_{3} \cdot v_{1} \\
l_{1} \cdot v_{2} & l_{2} \cdot v_{2} & l_{3} \cdot v_{2} \\
l_{1} \cdot v_{3} & l_{2} \cdot v_{3} & l_{3} \cdot v_{3}
\end{array}\right]\left[\begin{array}{l}
\xi_{1} \\
\xi_{2} \\
\xi_{3}
\end{array}\right] } \\
& +\left[\begin{array}{ll}
c \cdot v_{1}+h_{1} \cdot v_{1} \xi_{2} \xi_{3}+h_{2} \cdot v_{1} \xi_{3} \xi_{1}+h_{3} \cdot v_{1} \xi_{1} \xi_{2}+h_{4} \cdot v_{1} \xi_{1} \xi_{2} \xi_{3} \\
c \cdot v_{2}+h_{1} \cdot v_{2} \xi_{2} \xi_{3}+h_{2} \cdot v_{2} \xi_{3} \xi_{1}+h_{3} \cdot v_{2} \xi_{1} \xi_{2}+h_{4} \cdot v_{2} \xi_{1} \xi_{2} \xi_{3} \\
c \cdot v_{3}+h_{1} \cdot v_{3} \xi_{2} \xi_{3}+h_{2} \cdot v_{3} \xi_{3} \xi_{1}+h_{3} \cdot v_{3} \xi_{1} \xi_{2}+h_{4} \cdot v_{3} \xi_{1} \xi_{2} \xi_{3}
\end{array}\right]
\end{aligned}
$$


Applying the inverse relation (4.11) to this yields

Denoting

$$
\begin{aligned}
{\left[\begin{array}{l}
v_{1} \\
v_{2} \\
v_{3}
\end{array}\right]=} & {\left[\begin{array}{lll}
l_{1} \cdot v_{1} & l_{2} \cdot v_{1} & l_{3} \cdot v_{1} \\
l_{1} \cdot v_{2} & l_{2} \cdot v_{2} & l_{3} \cdot v_{2} \\
l_{1} \cdot v_{3} & l_{2} \cdot v_{3} & l_{3} \cdot v_{3}
\end{array}\right]\left[\begin{array}{lll}
l_{1} \cdot x_{1} & l_{2} \cdot x_{1} & l_{3} \cdot x_{1} \\
l_{1} \cdot x_{2} & l_{2} \cdot x_{2} & l_{3} \cdot x_{2} \\
l_{1} \cdot x_{3} & l_{2} \cdot x_{3} & l_{3} \cdot x_{3}
\end{array}\right]^{-1} } \\
& \times\left[\begin{array}{l}
x_{1}-\left(c \cdot x_{1}+h_{1} \cdot x_{1} \xi_{2} \xi_{3}+h_{2} \cdot x_{1} \xi_{3} \xi_{1}+h_{3} \cdot x_{1} \xi_{1} \xi_{2}+h_{4} \cdot x_{1} \xi_{1} \xi_{2} \xi_{3}\right) \\
x_{2}-\left(c \cdot x_{2}+h_{1} \cdot x_{2} \xi_{2} \xi_{3}+h_{2} \cdot x_{2} \xi_{3} \xi_{1}+h_{3} \cdot x_{2} \xi_{1} \xi_{2}+h_{4} \cdot x_{2} \xi_{1} \xi_{2} \xi_{3}\right) \\
x_{3}-\left(c \cdot x_{3}+h_{1} \cdot x_{3} \xi_{2} \xi_{3}+h_{2} \cdot x_{3} \xi_{3} \xi_{1}+h_{3} \cdot x_{3} \xi_{1} \xi_{2}+h_{4} \cdot x_{3} \xi_{1} \xi_{2} \xi_{3}\right)
\end{array}\right] \\
& +\left[\begin{array}{l}
c \cdot v_{1}+h_{1} \cdot v_{1} \xi_{2} \xi_{3}+h_{2} \cdot v_{1} \xi_{3} \xi_{1}+h_{3} \cdot v_{1} \xi_{1} \xi_{2}+h_{4} \cdot v_{1} \xi_{1} \xi_{2} \xi_{3} \\
c \cdot v_{2}+h_{1} \cdot v_{2} \xi_{2} \xi_{3}+h_{2} \cdot v_{2} \xi_{3} \xi_{1}+h_{3} \cdot v_{2} \xi_{1} \xi_{2}+h_{4} \cdot v_{2} \xi_{1} \xi_{2} \xi_{3} \\
c \cdot v_{3}+h_{1} \cdot v_{3} \xi_{2} \xi_{3}+h_{2} \cdot v_{3} \xi_{3} \xi_{1}+h_{3} \cdot v_{3} \xi_{1} \xi_{2}+h_{4} \cdot v_{3} \xi_{1} \xi_{2} \xi_{3}
\end{array}\right]
\end{aligned}
$$

$$
\left[\begin{array}{lll}
b_{1} \cdot v_{1} & b_{2} \cdot v_{1} & b_{3} \cdot v_{1} \\
b_{1} \cdot v_{2} & b_{2} \cdot v_{2} & b_{3} \cdot v_{2} \\
b_{1} \cdot v_{3} & b_{2} \cdot v_{3} & b_{3} \cdot v_{3}
\end{array}\right]=\left[\begin{array}{lll}
l_{1} \cdot v_{1} & l_{2} \cdot v_{1} & l_{3} \cdot v_{1} \\
l_{1} \cdot v_{2} & l_{2} \cdot v_{2} & l_{3} \cdot v_{2} \\
l_{1} \cdot v_{3} & l_{2} \cdot v_{3} & l_{3} \cdot v_{3}
\end{array}\right]\left[\begin{array}{ccc}
l_{1} \cdot x_{1} & l_{2} \cdot x_{1} & l_{3} \cdot x_{1} \\
l_{1} \cdot x_{2} & l_{2} \cdot x_{2} & l_{3} \cdot x_{2} \\
l_{1} \cdot x_{3} & l_{2} \cdot x_{3} & l_{3} \cdot x_{3}
\end{array}\right]^{-1},
$$

one can obtain

$$
\begin{aligned}
{\left[\begin{array}{l}
v_{1} \\
v_{2} \\
v_{3}
\end{array}\right]=} & {\left[\begin{array}{lll}
b_{1} \cdot v_{1} & b_{2} \cdot v_{1} & b_{3} \cdot v_{1} \\
b_{1} \cdot v_{2} & b_{2} \cdot v_{2} & b_{3} \cdot v_{2} \\
b_{1} \cdot v_{3} & b_{2} \cdot v_{3} & b_{3} \cdot v_{3}
\end{array}\right]\left[\begin{array}{l}
x_{1} \\
x_{2} \\
x_{3}
\end{array}\right] } \\
& +\left[\begin{array}{ll}
a \cdot v_{1}+g_{1} \cdot v_{1} \xi_{2} \xi_{3}+g_{2} \cdot v_{1} \xi_{3} \xi_{1}+g_{3} \cdot v_{1} \xi_{1} \xi_{2}+g_{4} \cdot v_{1} \xi_{1} \xi_{2} \xi_{3} \\
a \cdot v_{2}+g_{1} \cdot v_{2} \xi_{2} \xi_{3}+g_{2} \cdot v_{2} \xi_{3} \xi_{1}+g_{3} \cdot v_{2} \xi_{1} \xi_{2}+g_{4} \cdot v_{2} \xi_{1} \xi_{2} \xi_{3} \\
a \cdot v_{3}+g_{1} \cdot v_{3} \xi_{2} \xi_{3}+g_{2} \cdot v_{3} \xi_{3} \xi_{1}+g_{3} \cdot v_{3} \xi_{1} \xi_{2}+g_{4} \cdot v_{3} \xi_{1} \xi_{2} \xi_{3}
\end{array}\right]
\end{aligned}
$$

where

$$
\begin{aligned}
& a=c-\left(c \cdot x_{1}\right) b_{1}-\left(c \cdot x_{2}\right) b_{2}-\left(c \cdot x_{3}\right) b_{3}, \\
& g_{i}=h_{i}-\left(h_{i} \cdot x_{1}\right) b_{1}-\left(h_{i} \cdot x_{2}\right) b_{2}-\left(h_{i} \cdot x_{3}\right) b_{3}, \quad i=1,2,3 \text { and } 4 .
\end{aligned}
$$

Approximacing the differential relation between $x$ and $\xi$ by

$$
\begin{aligned}
& {\left[\begin{array}{l}
\frac{\partial}{\partial \xi_{1}} \\
\frac{\partial}{\partial \xi_{2}} \\
\frac{\partial}{\partial \xi_{3}}
\end{array}\right]=\left[\begin{array}{lll}
\frac{\partial x_{1}}{\partial \xi_{1}} & \frac{\partial x_{2}}{\partial \xi_{1}} & \frac{\partial x_{3}}{\partial \xi_{1}} \\
\frac{\partial x_{1}}{\partial \xi_{2}} & \frac{\partial x_{2}}{\partial \xi_{2}} & \frac{\partial x_{3}}{\partial \xi_{2}} \\
\frac{\partial x_{1}}{\partial \xi_{3}} & \frac{\partial x_{2}}{\partial \xi_{3}} & \frac{\partial x_{3}}{\partial \xi_{3}}
\end{array}\right]\left[\begin{array}{c}
\frac{\partial}{\partial x_{1}} \\
\frac{\partial}{\partial x_{2}} \\
\frac{\partial}{\partial x_{3}}
\end{array}\right] } \\
& \approx\left[\begin{array}{lll}
l_{1} \cdot x_{1} & l_{2} \cdot x_{1} & l_{3} \cdot x_{1} \\
l_{1} \cdot x_{2} & l_{2} \cdot x_{2} & l_{3} \cdot x_{2} \\
l_{1} \cdot x_{3} & l_{2} \cdot x_{3} & l_{3} \cdot x_{3}
\end{array}\right]\left[\begin{array}{c}
\frac{\partial}{\partial x_{1}} \\
\frac{\partial}{\partial x_{2}} \\
\frac{\partial}{\partial x_{3}}
\end{array}\right],
\end{aligned}
$$


differentiation with respect to the coordinate system $x$ is approximated by

$$
\left[\begin{array}{c}
\frac{\partial}{\partial x_{1}} \\
\frac{\partial}{\partial x_{2}} \\
\frac{\partial}{\partial x_{3}}
\end{array}\right] \approx\left[\begin{array}{lll}
l_{1} \cdot x_{1} & l_{2} \cdot x_{1} & l_{3} \cdot x_{1} \\
l_{1} \cdot x_{2} & l_{2} \cdot x_{2} & l_{3} \cdot x_{2} \\
l_{1} \cdot x_{3} & l_{2} \cdot x_{3} & l_{3} \cdot x_{3}
\end{array}\right]^{-t}\left[\begin{array}{c}
\frac{\partial}{\partial \xi_{1}} \\
\frac{\partial}{\partial \xi_{2}} \\
\frac{\partial}{\partial \xi_{3}}
\end{array}\right] .
$$

Thus, the differentiation of the bilinear and trilinear terms in $\xi$ w.r.t. $x$ is approximated as

$$
\begin{aligned}
{\left[\begin{array}{c}
\frac{\partial}{\partial x_{1}} \\
\frac{\partial}{\partial x_{2}} \\
\frac{\partial}{\partial x_{3}}
\end{array}\right]\left(\xi_{2} \xi_{3}\right) \approx } & {\left[\begin{array}{lll}
l_{1} \cdot x_{1} & l_{2} \cdot x_{1} & l_{3} \cdot x_{1} \\
l_{1} \cdot x_{2} & l_{2} \cdot x_{2} & l_{3} \cdot x_{2} \\
l_{1} \cdot x_{3} & l_{2} \cdot x_{3} & l_{3} \cdot x_{3}
\end{array}\right]^{-t}\left[\begin{array}{c}
\frac{\partial}{\partial \xi_{1}} \\
\frac{\partial}{\partial \xi_{2}} \\
\frac{\partial}{\partial \xi_{3}}
\end{array}\right]\left(\xi_{2} \xi_{3}\right) } \\
& =\left[\begin{array}{lll}
l_{1} \cdot x_{1} & l_{2} \cdot x_{1} & l_{3} \cdot x_{1} \\
l_{1} \cdot x_{2} & l_{2} \cdot x_{2} & l_{3} \cdot x_{2} \\
l_{1} \cdot x_{3} & l_{2} \cdot x_{3} & l_{3} \cdot x_{3}
\end{array}\right]^{-1}\left[\begin{array}{c}
0 \\
\xi_{3} \\
\xi_{2}
\end{array}\right],
\end{aligned}
$$

etc.

Applying these relations, the first derivatives of $\boldsymbol{v}$ w.r.t. $\boldsymbol{x}$ can be approximately obtained in terms of $\xi$. Furthermore, noting that polynomials in $\xi$ in the master element $\Omega_{M}$ can be explicitly integrated, the eleinent stiffness matrix due to the bilinear form $a_{Y e}\left(\cdot,{ }^{\prime}\right)$ can be approximately obtained without applying a quadrature rule. Because of this direct evaluation of integration less computing time is required to form the element stiffness matrix than in the case where a quadrature rule is used.

Now some examples of the homogenization method are introduced for 2D and 3D cases as well as the computation of the localized stress distribution. The above procedure was used for the 3D examples in order to reduce the computational time.

EXAMPLE 4.1 (Discontinuous fiber in plane elasticity). Consider a plane-stress linearlyelastic problem to obtain the homogenized elastic constants for the microscopic cell structure shown in Fig. 4.1. In this case many short boron fibers are periodically arranged in an aluminum matrix. Although the real cross-section of fibers is circular, in this example a rectangular cross section is assumed to construct an approximated plane stress problem. The dimensions $a=100 \mathrm{~mm}$ and $b=2 \mathrm{~mm}$ are assumed in order to have $50 \%$ fiber volume in this composite material. This implies that $\varepsilon=10 \mathrm{~mm}$, where $\varepsilon$ is the parameter in the perturbation of $u^{\varepsilon}$, while the size of the cell is defined by the proportionality relation $1: 0.02$. Figure 4.2 shows the homogenized elastic constants for various lengths of short fibers. In this case, the gap of short fibers is held as a constant while the length of short fibers are varying. It is clear that the fiber length affects only $D_{111}$ which represents the elastic rigidity of the composite in 

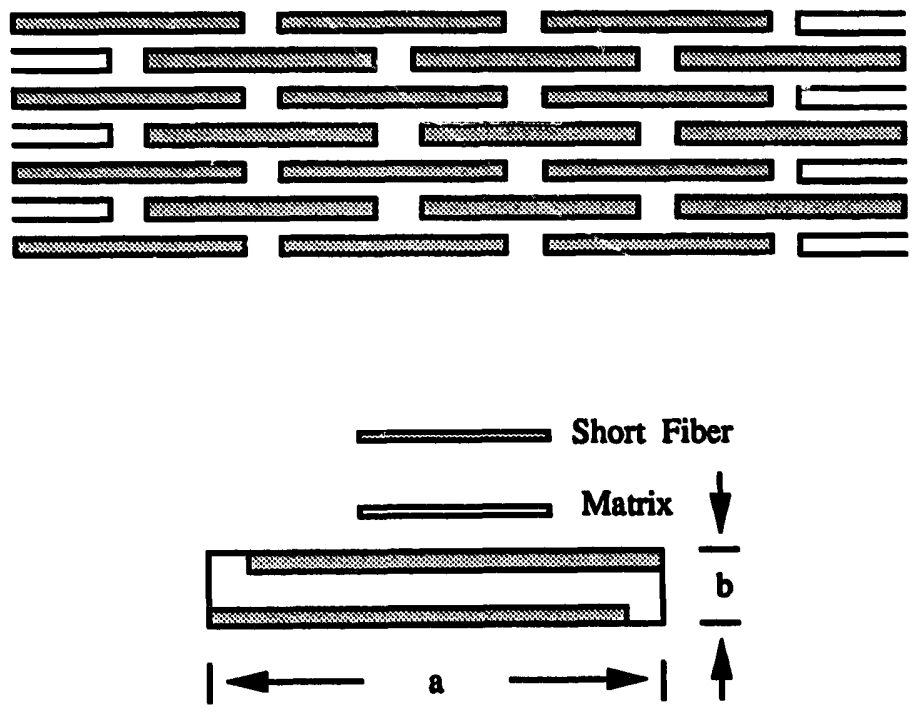

Basic Cell

\title{
Boron Fiber $\quad \mathrm{E} 1111=538.492 \mathrm{GPa}$ $\mathrm{E} 1122=230.769 \mathrm{GPa}$ $\mathrm{E} 1212=153.846 \mathrm{Gpa}$
}

\author{
Al Matrix E1111 $=108 \mathrm{GPa}$ \\ $\mathrm{E} 1122=54 \mathrm{GPa}$ \\ $\mathrm{E} 1212=27 \mathrm{GPa}$
}

Fig. 4.1. A plane stress model of a short fiber reinforced composite.

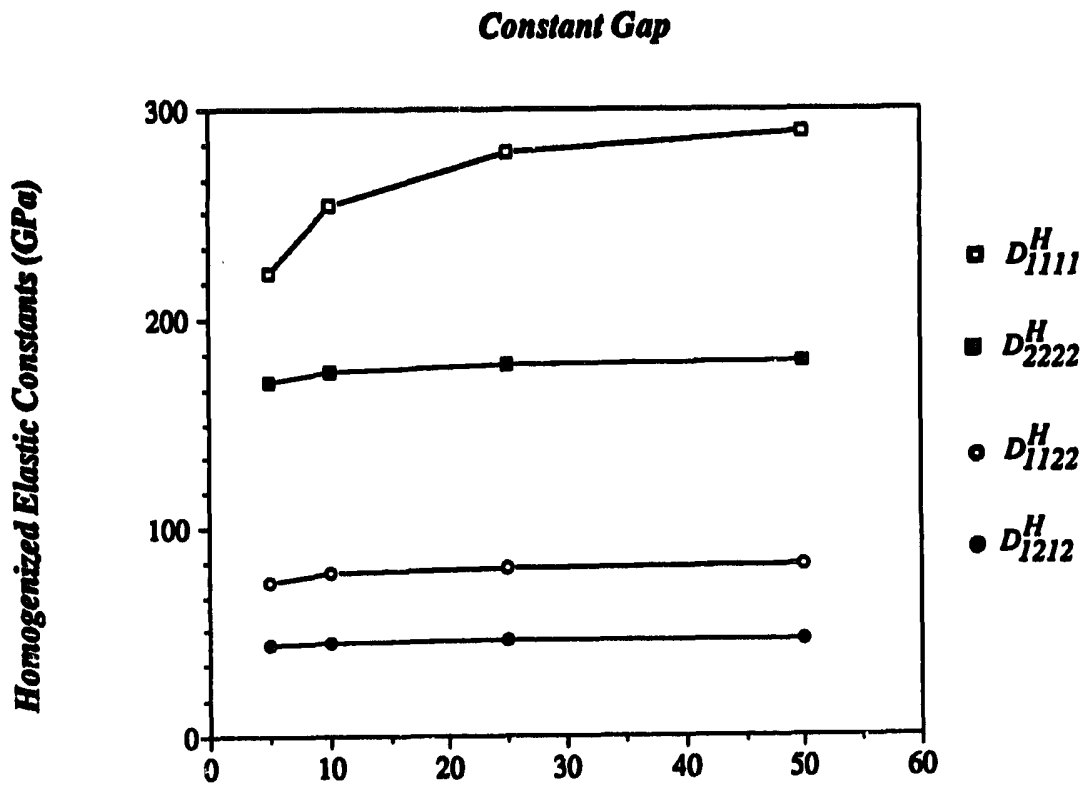

Ratio Fuber Length/Fiber Widh

Fig. 4.2. Homogenized elastic constants of a composite plate reinforced by boron short fibers (constant gap). 
the fiber direction. It is also clear that if the fiber length reaches to $a$ comparable value of the gap $0.2 \mathrm{~mm}$, the rigidity in the fiber direction is significantly reduced. However, if the gap is reduced proportionally to the fiber length, the homogenized elastic constants are almost constant, as shown in Fig. 4.3. Here, the gap is $10 \%$ of the fiber length. This means that if the gap can be reduced proportionally to the fiber length, the fiber length does not greatly affect the homogenized elastic constants. This may indicate that the fiber length should be decided by failure criteria of fibers, matrix and their bonding interface.

EXAMPLE 4.2 (Continuous fiber in three-dimensional elasticity). Consider a boron fiber reinforced composite material. Fibers are assumed to be unidirectional, and their volume fraction is $40 \%, 50 \%$ and $60 \%$, respectively, in an aluminum matrix as shown in Fig. 4.4. The homogenized elastic constants are obtained as in Fig. 4.5. The values of Young's moduli $E_{1}$, $E_{2}$ and $E_{3}$ are compared with those obtained experimentally in [21]. It is clear that Young's moduli in the fiber direction is very close to that obtained by the homogenization method.

EXAMPLE 4.3 (Honeycomb structures). Consider a honeycomb structure with and without shear panels on the top and bottom surfaces, its microstructure is given in Fig. 4.6. This example tries to show the effect of shear panels in a honeycomb structure. The model used is based on a unit cell with dimension ratios

$$
\text { length } / \text { width } / \text { height } / \text { thickness }=0.7696 / 0.866 / 1.5 / 0.1 \text {. }
$$

\section{Proportional Gap}

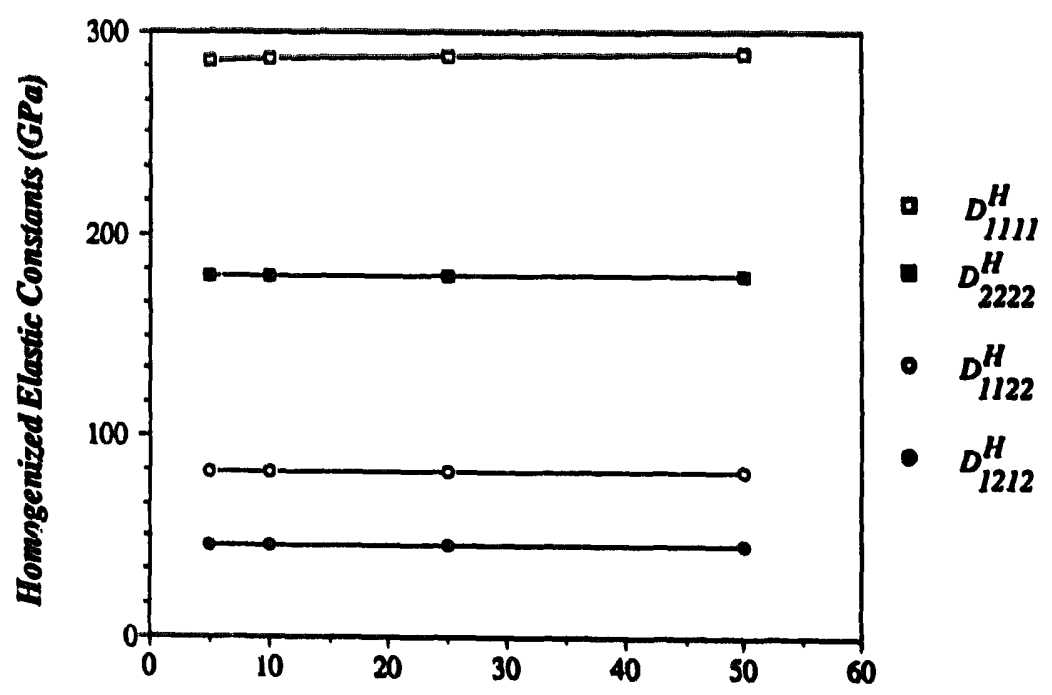

\section{Ratio Fiber Length/Fiber Widh}

Fig. 4.3. Homogenized elastic constants of a composite plate reinforced by boron short fibers (proportional gap). 


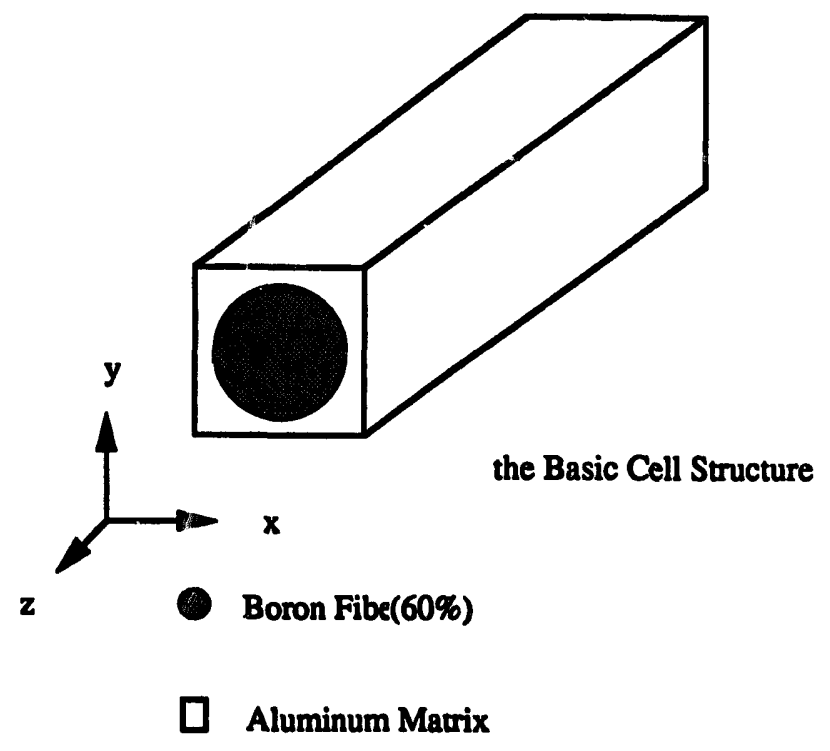

Fig. 4.4. Boron fiber reinforced composite material.

For an aluminum honeycomb, the homogenized elastic constants are computed for several different thicknesses of the additional shear panels. The results are shown in Fig. 4.7 and the characteristic deformations for a particular case are as in Fig. 4.8. The results for $D_{2222}$ and $D_{2233}$ are omitted because they behave similarly to $D_{3333}$ and $D_{1133}$, respectively. As expected, these panels reinforce the in plane rigidity $\left(D_{1111}, D_{1212}\right)$ but have few influence on the out of plane rigidity $\left(D_{3333}, D_{1133}\right)$.

\section{Boron/6061Al Composite}

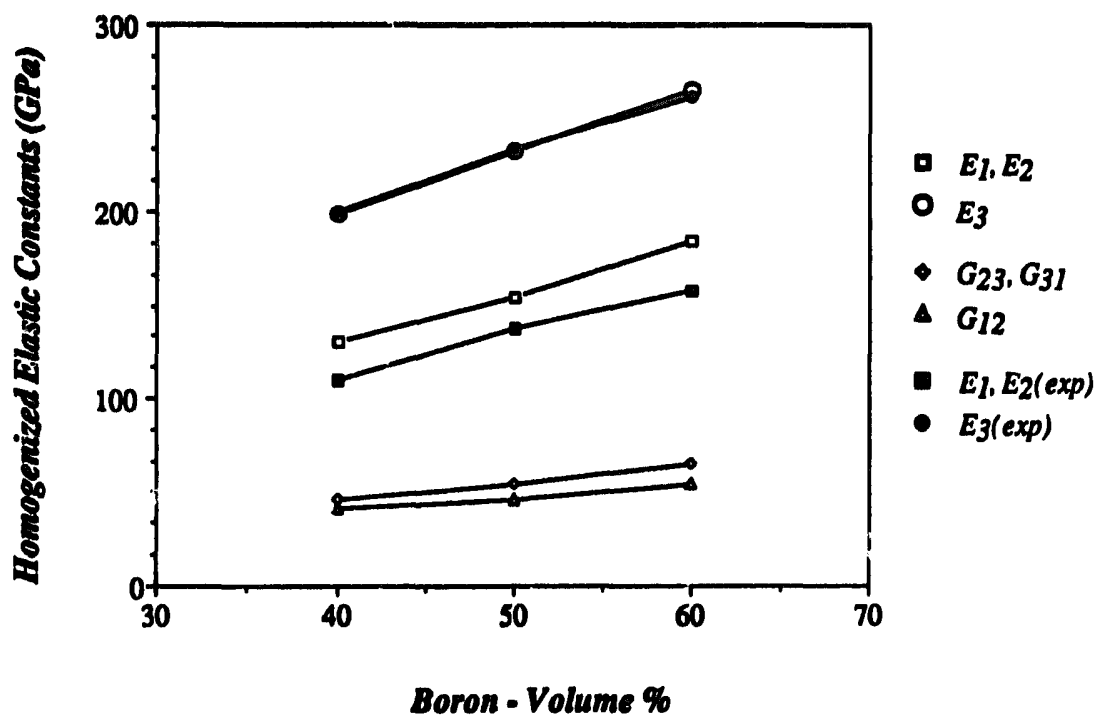

Fig. 4.5. Homogenized elastic constants of boron fiber reinforced aluminium. 


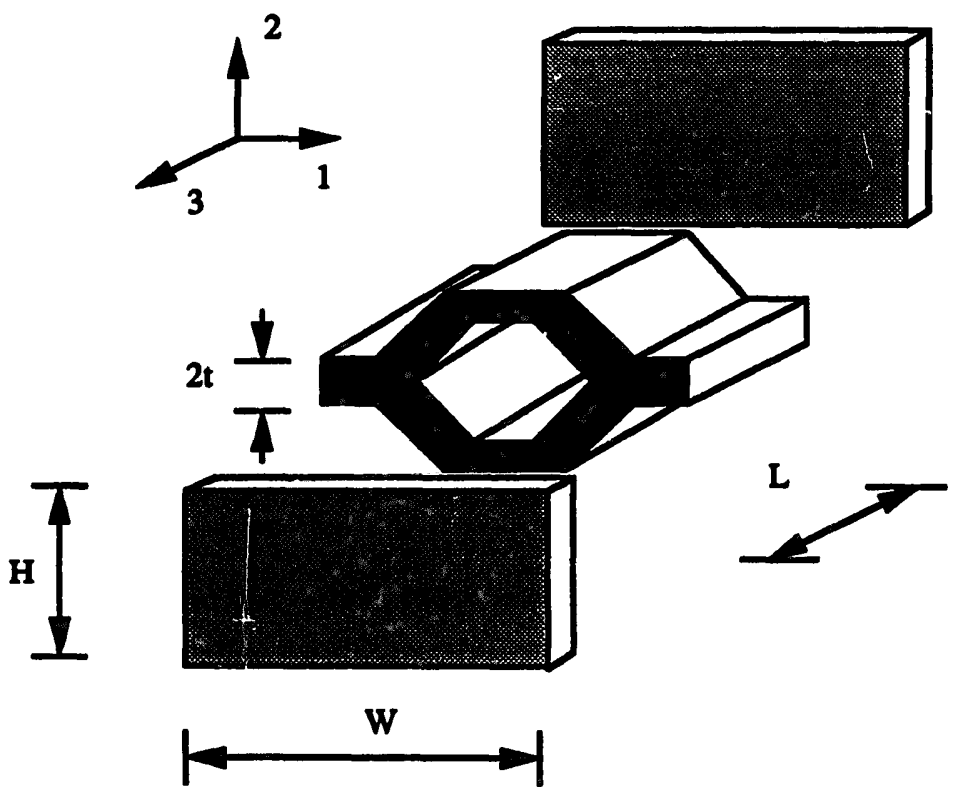

Fig. 4.6. Microstructure of a honeycomb.

EXAMPLE 4.4 (Woven fiber reinforced composites). The fourth example is for a woven fiber reinforced composite material whose finite element model is shown in Fig. 4.9. In this case, fibers are not straight, but woven three-dimensionally in both directions of a plane so that the elastic modulus in the thickness direction, which is transverse to the plane, is different from the moduli in the plane directions. We assume boron fibers and Al matrix are used in Example 4.1, although it may not be realistic. The homogenized elastic constants are obtained as

Honey-Comb with Layers

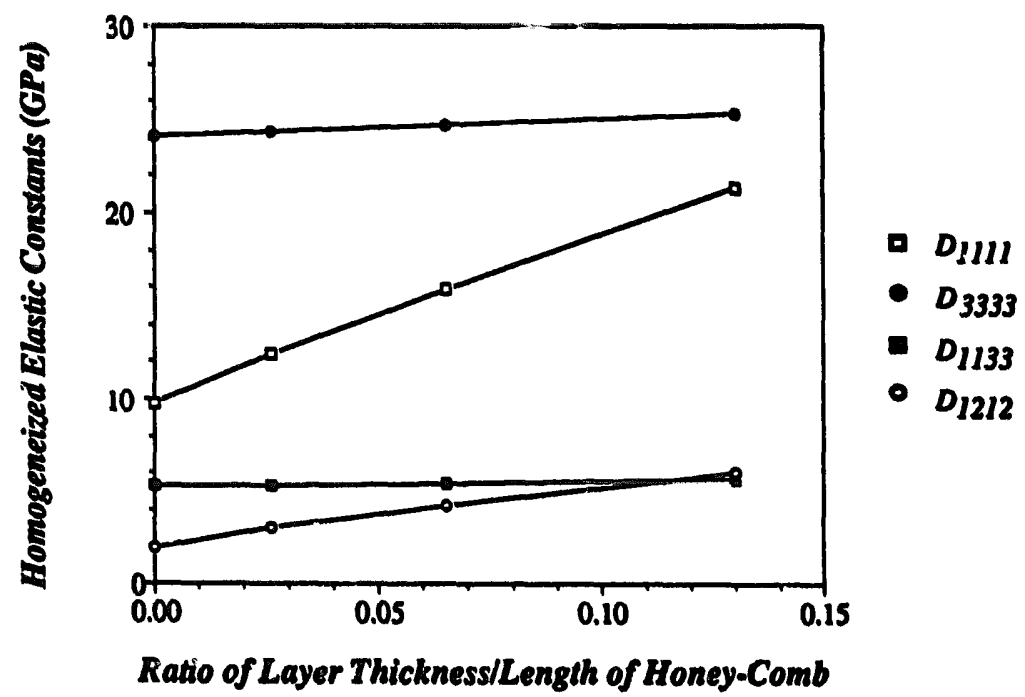

Fig. 4.7. Homogenized elastic constants of honeycombed structures. 

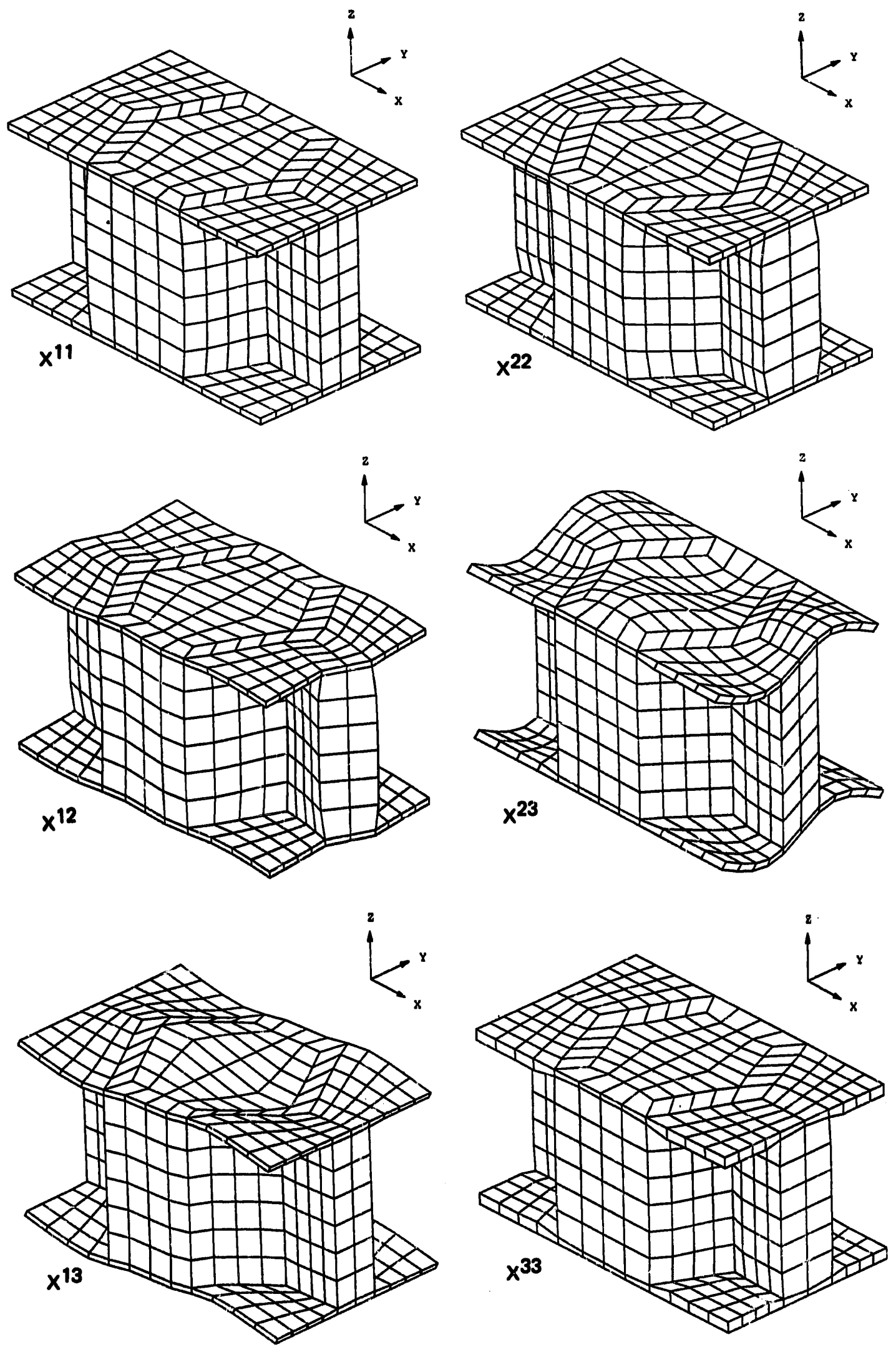

Fig. 4.8. Characteristic deformations $\left(x^{11}, x^{12}, x^{13}, x^{22}, x^{23}, x^{33}\right)$. 

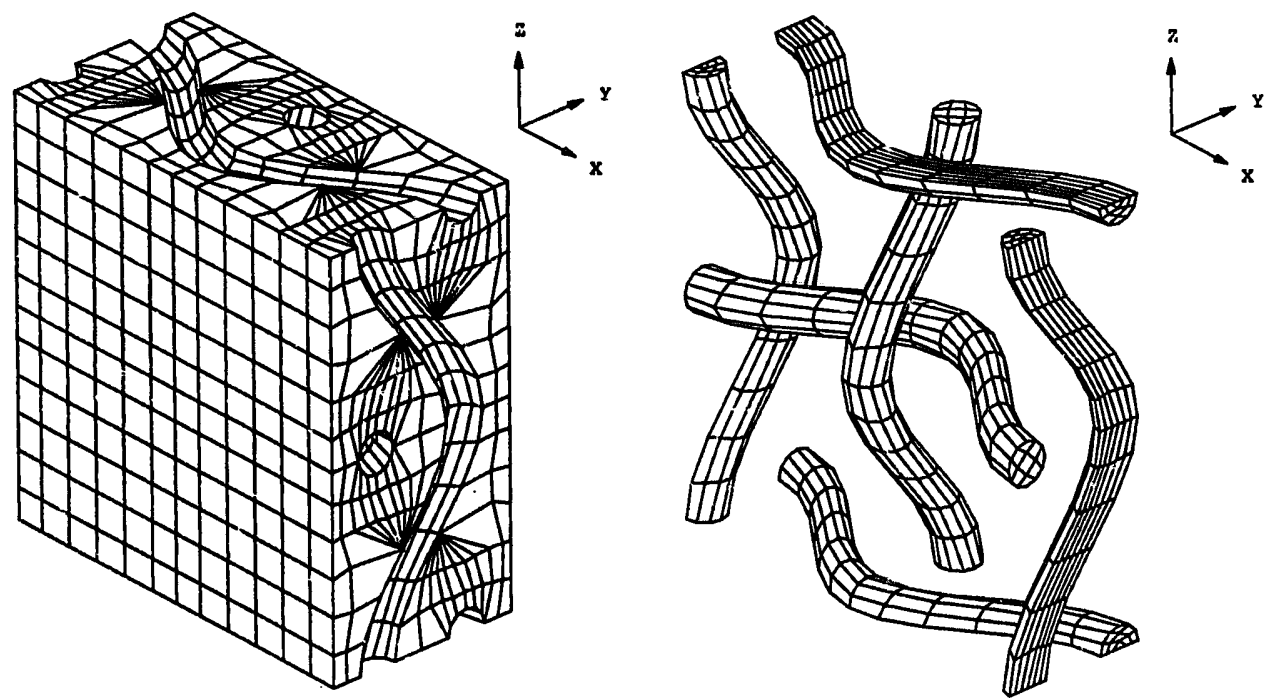

Fig. 4.9. Woven fiber reinforced composite (matrix and fiber finite element method).

$$
\begin{array}{lll}
D_{1111}=119.94 \mathrm{GPa}, & D_{1122}=57.84 \mathrm{GPa}, & D_{1133}=57.03 \mathrm{GPa}, \\
D_{2222}=116.66 \mathrm{GPa}, & D_{2233}=57.84 \mathrm{GPa}, \\
& D_{3333}=119.94 \mathrm{GPa}, \\
D_{2323}=30.29 \mathrm{GPa}, & D_{1313}=29.77 \mathrm{GPa}, & D_{1212}=30.29 \mathrm{GPa},
\end{array}
$$

by solving the homogenization problem using 3240 HEXA8 elements. The characteristic deformations are given in Fig. 4.10.

Now, once the homogenized elastic constants and the 'residual' stresses are computed, the macroscopic problem is solved to determine the homogenized response $u^{0}$ by solving the discrete problem (3.41):

$$
\text { Find } u^{0^{h}} \in V_{\Omega}^{h} \text {, such that } a^{H}\left(u^{0^{h}}, v^{h}\right)=f^{H}\left(v^{h}\right) \quad \forall v^{h} \in V_{\Omega}^{h} \text {. }
$$

After solving the macroscopic problem, it is necessary to compute the displacement and stress fields in the microscopic structure using the expansions (2.58) and (2.59):

and

$$
u^{H^{01}}(x)=u^{H^{0}}(x)-\varepsilon\left(x^{H^{k l}}(x, y) \frac{\partial u_{k}^{H^{0}}(x)}{\partial x_{l}}+\psi^{H}(x, y)-\tilde{u}^{H^{1}}(x)\right)
$$

$$
\sigma_{i j}^{H^{0}}(x, y)=\left(E_{i j k l}(x, y)-E_{i j p m}(x, y) \frac{\partial \chi_{p}^{H^{k l}}(x, y)}{\partial y_{m}}\right) \frac{\partial u_{k}^{H^{0}}(x)}{\partial x_{l}}-E_{i j k l}(x, y) \frac{\partial \psi_{k}^{H}(x, y)}{\partial y_{l}} \text {. }
$$



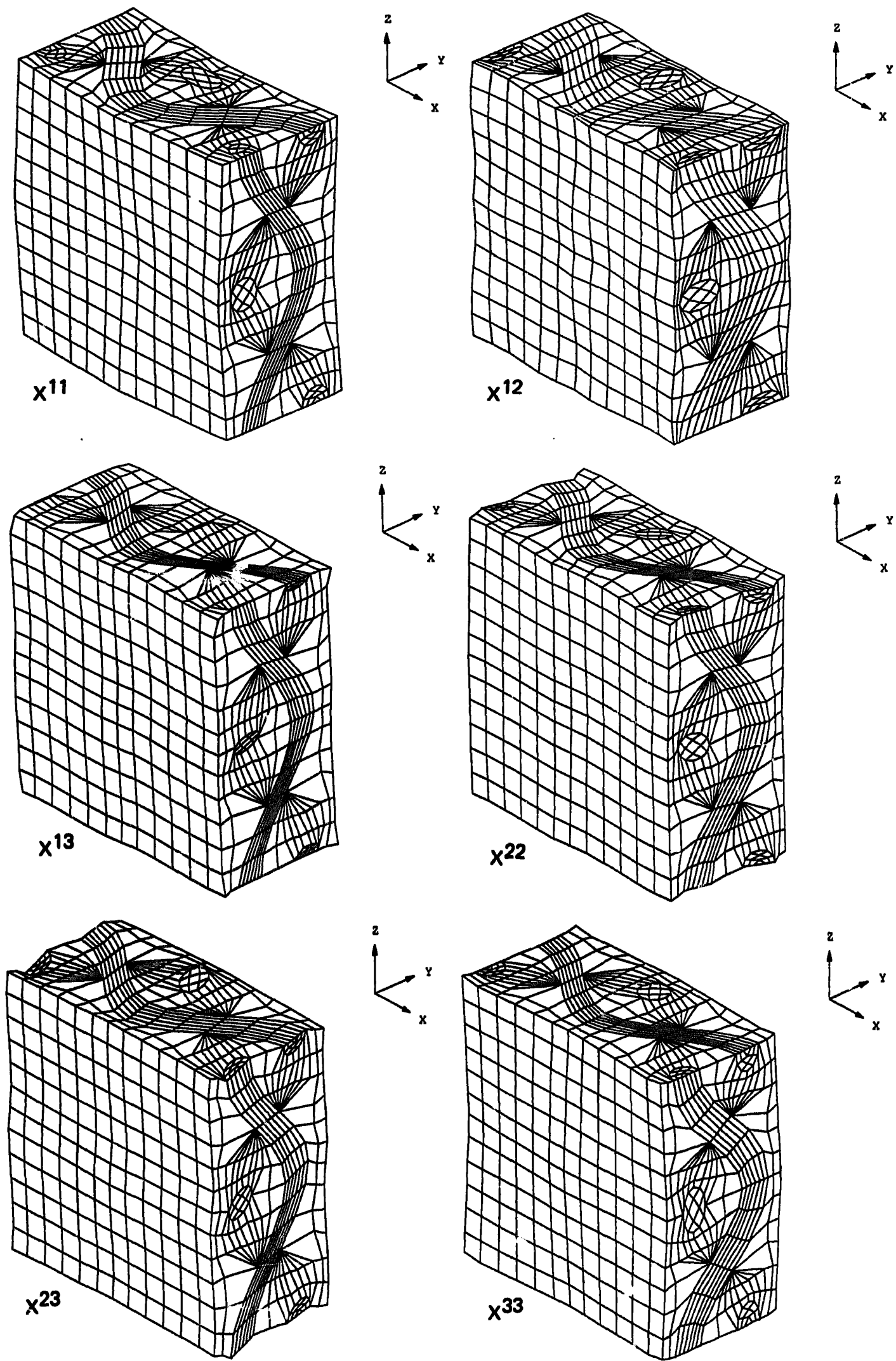

Fig. 4.10. Characteristic deformations $\left(x^{11}, x^{12}, x^{13}, x^{22}, x^{23}, x^{33}\right)$. 
In POSTMAT, by specifying the point $x$ in a specified finite element $\Omega_{e}$ in the macroscopic domain, $u^{H^{0}}(x)$ is computed by interpolating its nodal values as well as its first derivatives in the macroscopic coordinates $x$. Then, if the material constants are obtained by the homogenization method, the approximations of the stresses $\sigma_{i j}^{H^{0}}(x, y)$ are computed using the characteristic deformations $\chi_{p}^{H^{k l}}(x, y)$ and the residual stresses $\psi_{k}^{H}(x, y)$ in the finite element model of the cell domain $\boldsymbol{Y}$.

EX AMPLE 4.5 (Continuation of Example 4.2). Consider a lamina of boron fiber reinforced aluminium with a microscopic structure as shown in Fig. 4.4, and let a laminate consist of two laminae whose fiber orientation is inclined +45 and -45 degrees w.r.t. the longitudinal direction as shown in Fig. 4.11. Also, let the length, width and thickness of the laminate be 4, 1 and 0.2 , respectively. Then, applying the homogenized elastic constants for $60 \%$ of boron volume fraction, one solves a macroscopic stress analysis problem of the laminate. Assuming a force distribution on the half portion of the free end as shown in Fig. 4.11, this stress analysis, problem (4.17), is solved by the finite element method using $8 \times 4 \times 4$ uniform HEXA8 elements. In order to avoid shear locking one applies the directional reduced integration method with hourglass control, as previously described. The approximated stress fields are computed in each lamina, and also at the microscopic level for points $A, B$ and $C$. For these points, the microscale Von Mises equivalent stress contour lines are shown in Figs. 4.12, 4.13 and 4.14.

EXAMPLE 4.6 (Continuation of Example 4.3). Consider a macroscopic structure as shown in Fig. 4.15 with an aluminium honeycomb microscale structure as shown in Fig. 4.6 and the same dimensions and load conditions as in Example 4.5. Similarly, after solving the macroscopic stress analysis problem using HEXA8 elements with the directional reduced integration method with hourglass control, the microscopic stress fields are computed at points $A, B$ and $C$ and the corresponding Von Mises equivalent stress contour lines shown in Figs. 4.16, 4.17 and 4.18 , respectively.

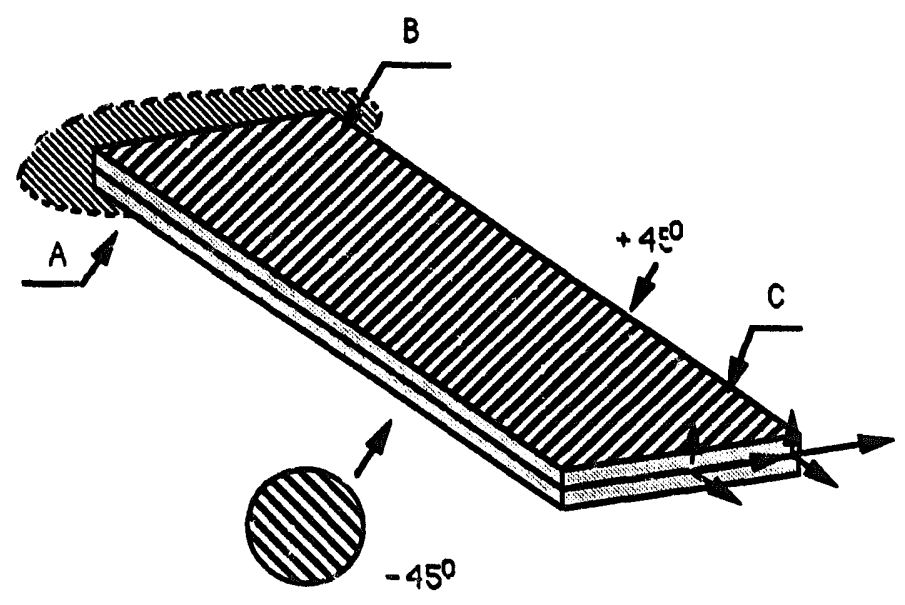

Fig. 4.11. A macroscopic stress analysis problem of a boron/AL laminate. 

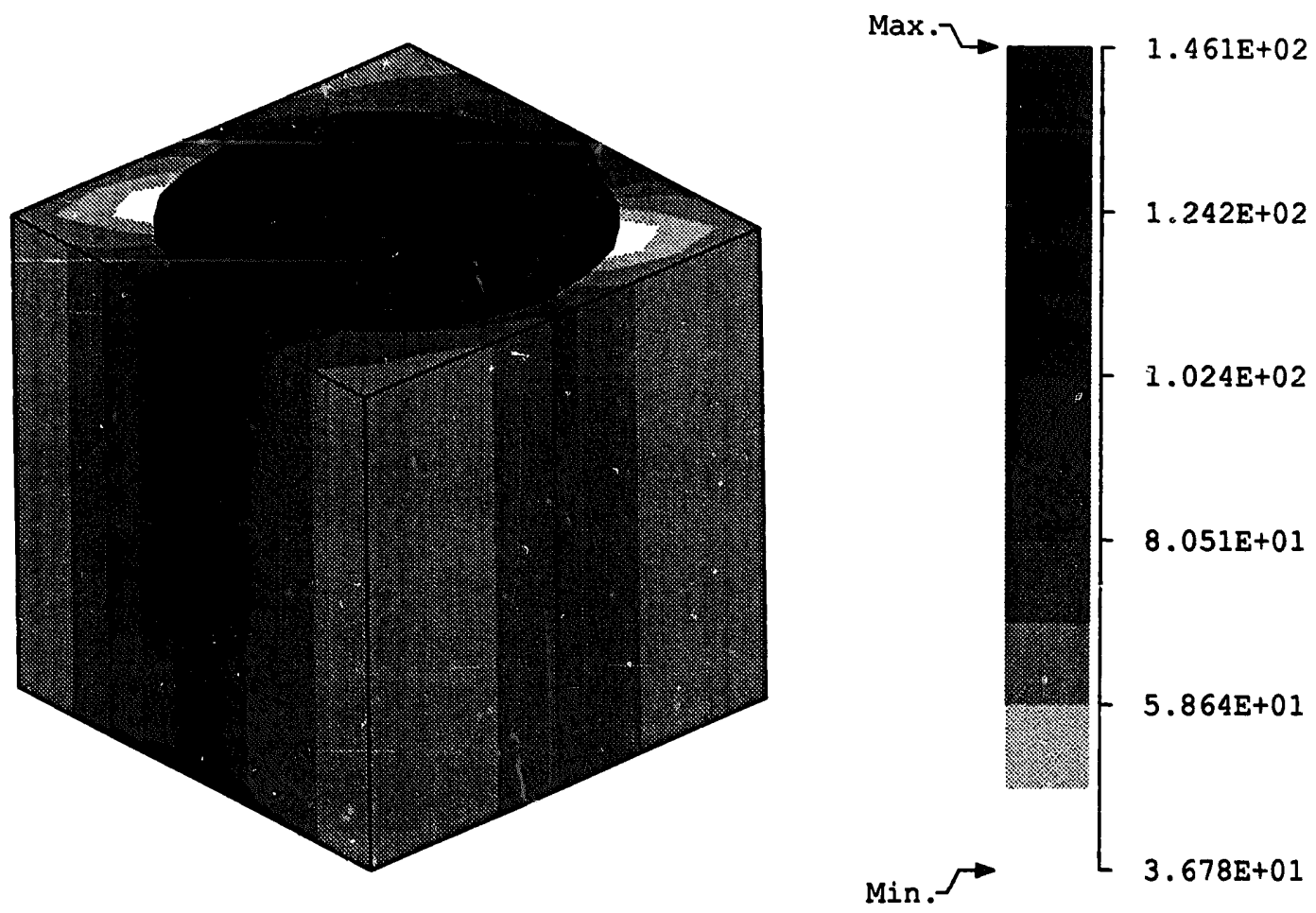

Fig. 4.12. Local distribution of equivalent Von Mises stress at point $\boldsymbol{A}$.
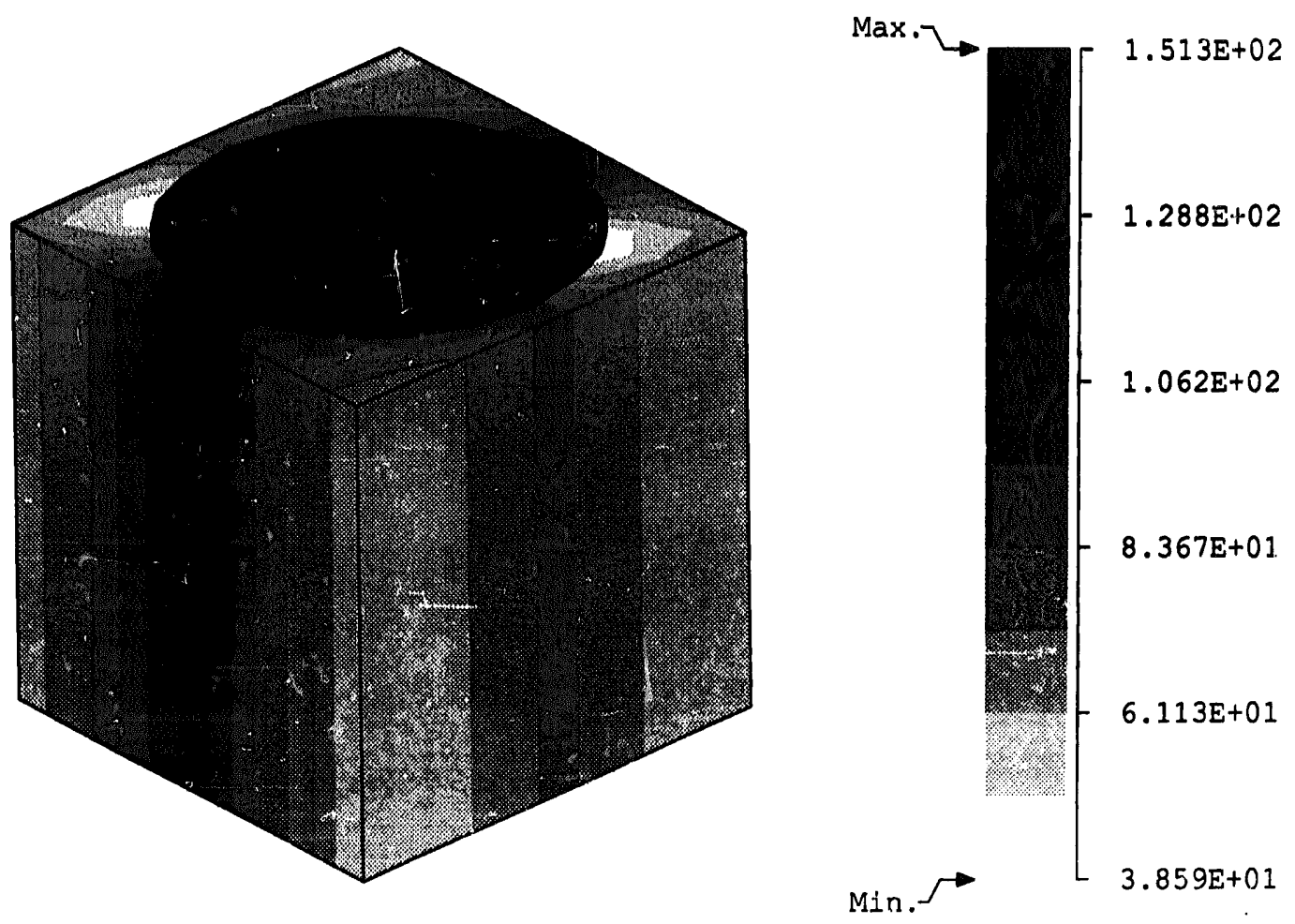

Fig. 4.13. Local distribution of equivalent Von Mises stress at point $B$. 

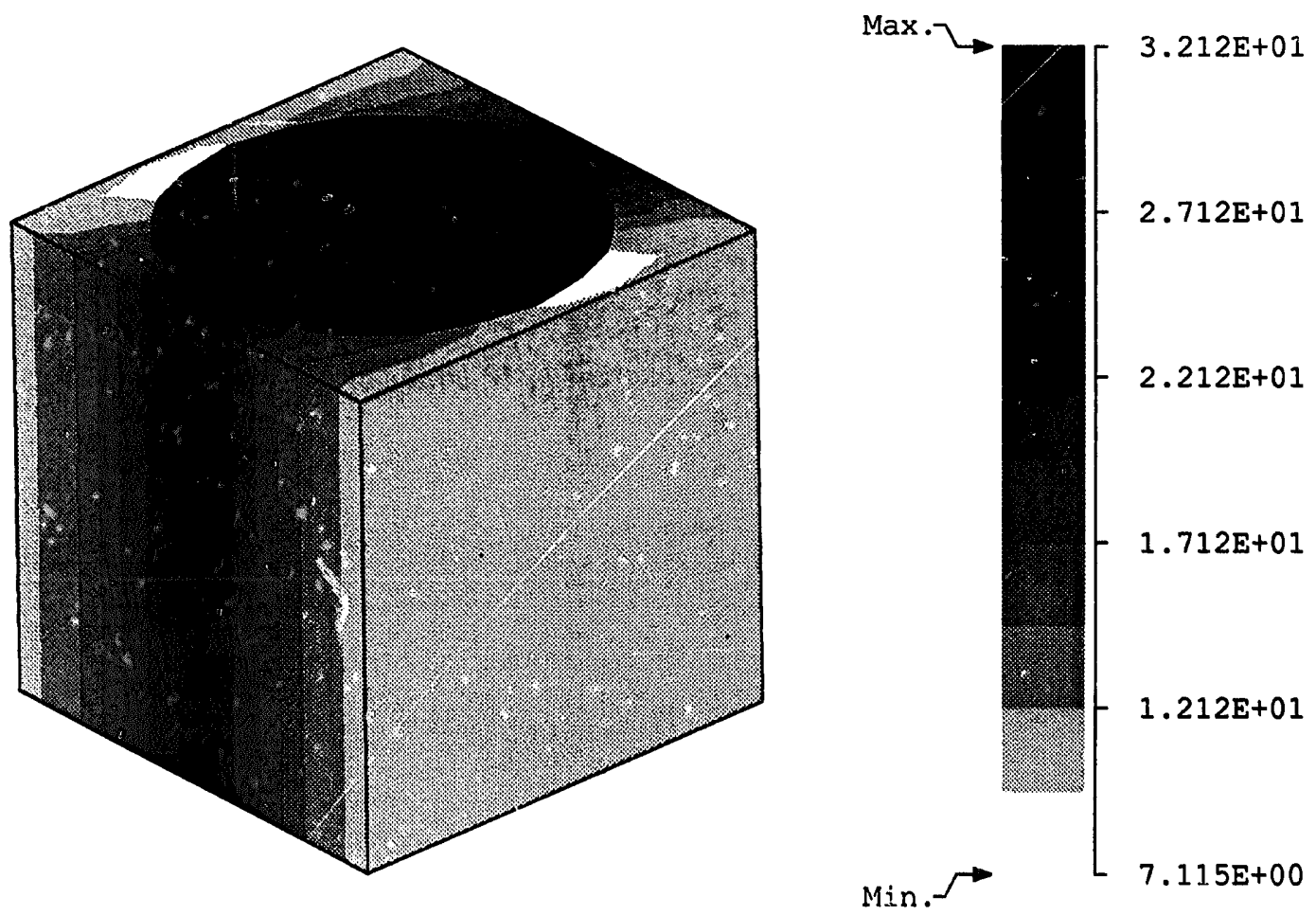

Fig. 4.14. Local distribution of equivalent Von Mises stress at point $C$.

\section{Application of the adaptive finite element method}

A general concern arising from the use of the finite element method is the accuracy of the solutions obtained. The main problem concerns the accuracy of the finite element solutions and how to improve them. A fairly standard technique to overcome this problem is the use of adaptive finite element methods. The initial ideas of these adaptive methods appeared in the late 60's (see, for example, [22]) and, in the 70's, Babuška, Rheinboldt and their colleagues [23-26] introduced their fundamental concepts and techniques together with a posteriori error estimates.

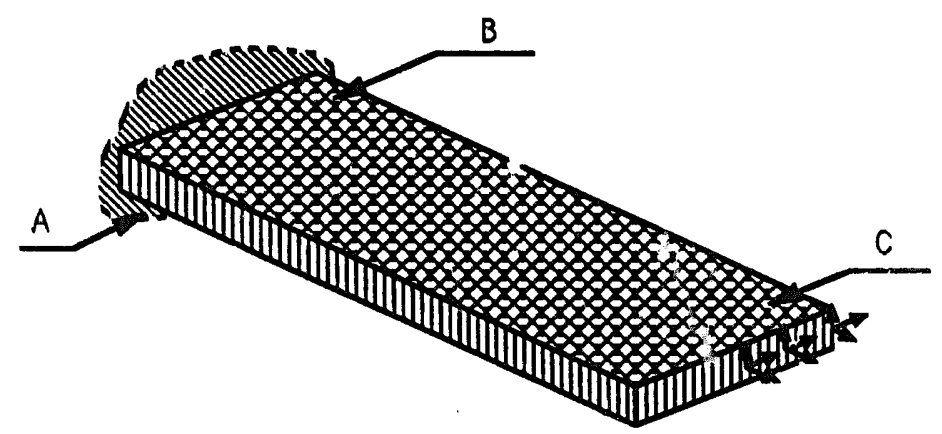

Fig. 4.15. A macroscopic stress analysis problem of a honeycomb plate. 

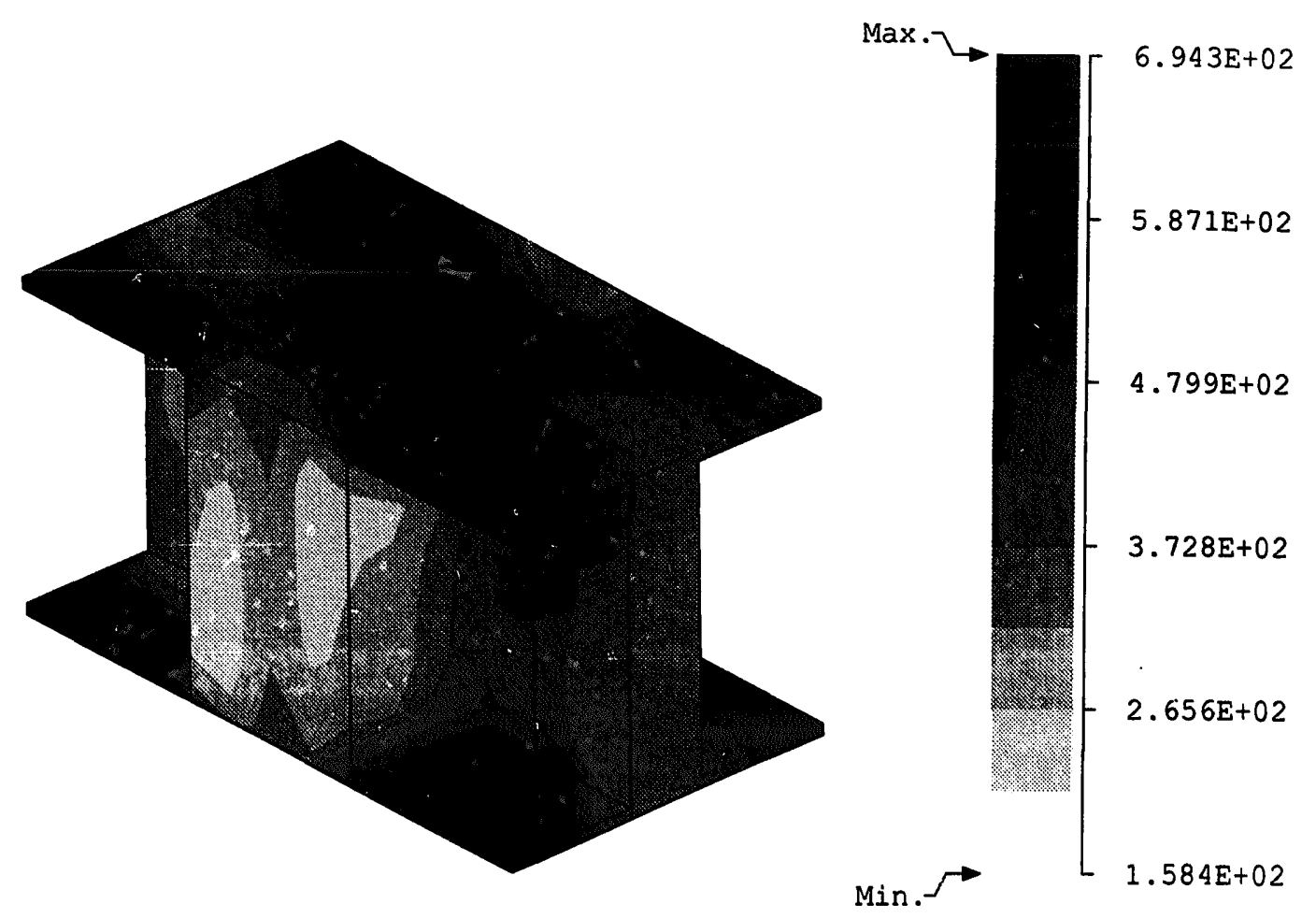

Fig. 4.16. Local distribution of equivalent Von Mises stress at point $A$.
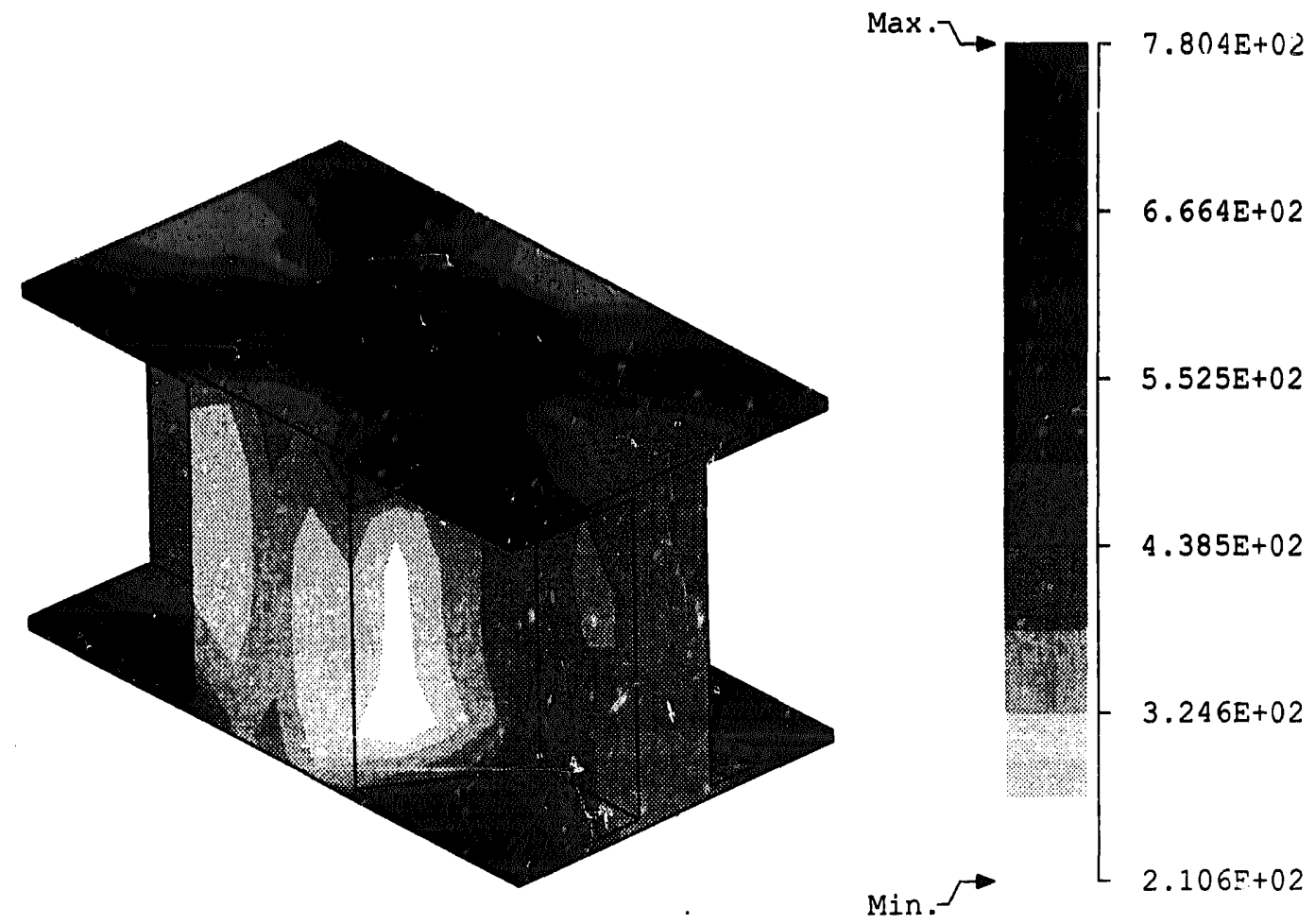

Fig. 4.17. Local distribution of equivalent Von Mises stress at point $B$. 


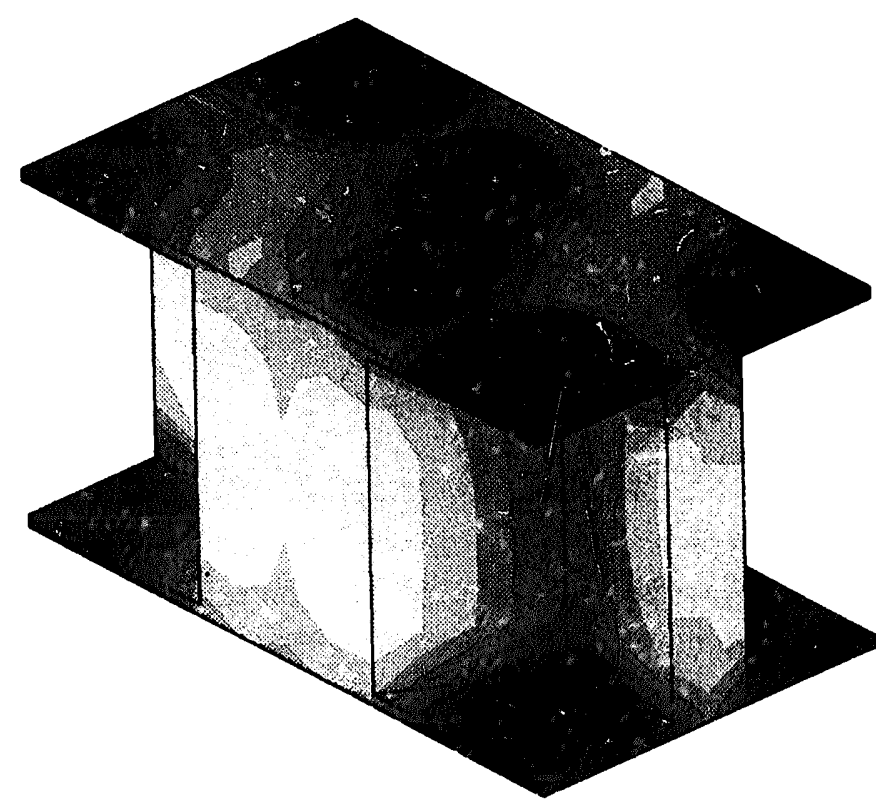

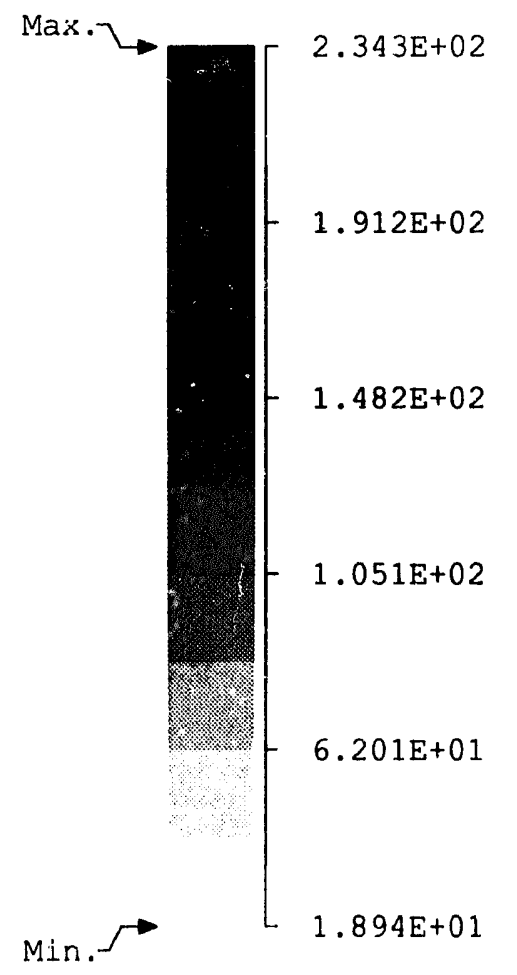

Fig. 4.18. Local distribution of equivalent Von Mises stress at point $C$.

The main idea of adaptive methods is to predict the amount of approximation error using only the finite element approximation, to modify the finite element mesh such that the approximation error is reduced with a minimum effort. These methods are traditionally classified with respict to the type of mesh modification: $r$-methods for node relocation, $\boldsymbol{h}$-methods for finite element refinement, and $\boldsymbol{p}$-methods for higher order polynomial interpolation. For any of these methods there are several different approaches usually related with the choice of error approximation and/or the method of implementation (see, for example, [27-29]). Details on these methods can be found in the literature mentioned before and references cited therein.

In the previous sections the homogenized elastic constants are computed using the finite element method with, consequently, the same accuracy concerns as above.

The first question to consider is whether the application of adaptive methods is necessary in determining the homogenized constants. If the values of the homogenized elastic constants are insensitive to finite element discretization, the use of adaptive methods is not important. To adciress this question, we reexamine the result of the convergence study of the finite element approximation given in Example 3.1. The base cell involves two different isotropic materials whose elastic moduli are significantly different. In this case, although the convergence rate is the same as the one predicted from the theory developed in Section 3, rather coarse meshes cannot provide accurate resuits. In other words, to achieve sufficiently accurate results, very refined finite eleniert meshes musi be used if uniform refinement is assumed. This indicates that application of adaptive methods would significantly improve the accuracy of finite element 
approximations without increasing too greatly the number of degrees of freedom of the finite element mesh.

The second question to consider, after realizing the need for adaptive methods, is what kind of a posteriori error measure should be used. For this one recalls the a priori error estimate of the finite element approximation of the homogenized elastic constants from (3.22):

$$
D_{i j k l}^{H}-D_{i j k l}=a_{Y}\left(\chi^{H^{i j}}-\chi^{i j}, \chi^{H^{k l}}-\chi^{k l}\right),
$$

where the finite element approximation of the characteristic deformations in the base cell satisfies the a priori error estimate

$$
\left\{a_{Y}\left(\chi^{H^{i j}}-\chi^{i j}, \chi^{H^{i j}}-\chi^{i j}\right\}^{1 / 2} \leqslant\left\{a_{Y}\left(v^{H}-\chi^{i j}, v^{H}-\chi^{i j}\right)\right\}^{1 / 2} \quad \forall v^{H} \in V_{Y}^{H},\right.
$$

that is, taking $v^{H}=\chi_{I}^{i j}$ yields

$$
\left\{a_{Y}\left(x^{H^{i j}}-x^{i j}, x^{H^{i j}}-\chi^{i j}\right)\right\}^{1 / 2} \leqslant\left\{a_{Y}\left(\chi_{I}^{i j}-{ }^{1} \chi^{i j}, \chi_{I}^{i j}-{ }^{1} \chi^{i j}\right)\right\}^{1 / 2} \leqslant C H^{\mu}\left\|^{1} \chi^{i j}\right\|_{l, Y} .
$$

Following Diaz et al. [27, 28], these two a priori error estimates yield an error measure for the characteristic deformations, in each finite element $\boldsymbol{Y}_{e}$, defined by

$$
E_{e}^{i j}=a_{Y e}\left(\chi_{I}^{i j}-{ }^{1} \chi^{i j}, \chi_{i}^{i j}-{ }^{1} \chi^{i j}\right)^{1 / 2}
$$

and, consequently, the error measure for the homogenized elastic constants in each finite element $\boldsymbol{Y}_{e}$ is defined by

$$
E_{e}^{i j k l}=E_{e}^{i j} E_{e}^{k l}
$$

If (5.5) is applied, different adapted finite element meshes are obtained for independent combinations of $i, j, k, l$ for the homogenized elastic constants $D_{i j k l}$. Thus, it is recommended to use another error measure,

$$
E_{e}=\operatorname{Max}_{i, j, k, l=1,2,3} E_{e}^{i j k l},
$$

which yields a unique adapted finite element mesh for all of the characteristic deformations and independent combinations of indices $i, j, k, l$ of the homogenized elastic constants.

In any case, error measures $E_{e}$ are written in the form of interpolation errors of the characteristic deformations. If HEXA8 elements, 8 node solid elements, are applied to solve three-dimensional problems in their finite element approximations, the interpolation error of a function $g$ which is twice differentiable in the master element is given by

$$
g_{\mathrm{I}}-g=\frac{1}{2}\left[\left(1-r^{2}\right) \frac{\partial^{2} g}{\partial r^{2}}+\left(1-s^{2}\right) \frac{\partial^{2} g}{\partial s^{2}}+\left(1-t^{2}\right) \frac{\partial^{2} g}{\partial t^{2}}\right] \text { at }(r, s, t) \in \Omega_{\mathrm{M}},
$$

and then 
and

$$
\begin{aligned}
& \frac{\partial\left(g_{1}-g\right)}{\partial r}=\frac{1}{2}\left\{-2 r \frac{\partial^{2} g}{\partial r^{2}}+\left(1-s^{2}\right) \frac{\partial^{3} g}{\partial s^{2} \partial r}+\left(1-t^{2}\right) \frac{\partial^{3} g}{\partial t^{2} \partial r}\right\}, \\
& \frac{\partial\left(g_{1}-g\right)}{\partial s}=\frac{1}{2}\left\{\left(1-r^{2}\right) \frac{\partial^{3} g}{\partial r^{2} \partial s}-2 s \frac{\partial^{2} g}{\partial s^{2}}+\left(1-t^{2}\right) \frac{\partial^{3} g}{\partial t^{2} \partial s}\right\}
\end{aligned}
$$

$$
\frac{\partial\left(g_{1}-g\right)}{\partial t}=\frac{1}{2}\left\{\left(1-r^{2}\right) \frac{\partial^{3} g}{\partial r^{2} \partial t}+\left(1-s^{2}\right) \frac{\partial^{3} g}{\partial s^{2} \partial t}-2 t \frac{\partial^{2} g}{\partial t^{2}}\right\} \text {. }
$$

Note that

and

$$
\left[\begin{array}{l}
\frac{\partial\left(g_{1}-g\right)}{\partial x} \\
\frac{\partial\left(g_{1}-g\right)}{\partial y} \\
\frac{\partial\left(g_{1}-g\right)}{\partial z}
\end{array}\right]=\left[\begin{array}{lll}
\frac{\partial x}{\partial r} & \frac{\partial y}{\partial r} & \frac{\partial z}{\partial r} \\
\frac{\partial x}{\partial s} & \frac{\partial y}{\partial s} & \frac{\partial z}{\partial s} \\
\frac{\partial x}{\partial t} & \frac{\partial y}{\partial t} & \frac{\partial z}{\partial t}
\end{array}\right]^{-1}\left[\begin{array}{l}
\frac{\partial\left(g_{\mathrm{i}}-g\right)}{\partial r} \\
\frac{\partial\left(g_{1}-g\right)}{\partial s} \\
\frac{\partial\left(g_{1}-g\right)}{\partial t}
\end{array}\right]
$$

$$
\begin{aligned}
\frac{\partial^{2} g}{\partial r^{2}}= & \frac{\partial\left(\frac{\partial x \partial g}{\partial r \partial x}+\frac{\partial y \partial g}{\partial r \partial y}+\frac{\partial z \partial g}{\partial r \partial r}\right)}{\partial r} \\
= & \left(\frac{\partial x}{\partial r}\right)^{2} \frac{\partial^{2} g}{\partial x^{2}}+\left(\frac{\partial y}{\partial r}\right)^{2} \frac{\partial^{2} g}{\partial y^{2}}+\left(\frac{\partial z}{\partial r}\right)^{2} \frac{\partial^{2} g}{\partial z^{2}} \\
& +2\left(\frac{\partial x \partial y \partial^{2} g}{\partial r \partial r \partial x \partial y}+\frac{\partial x \partial z \partial^{2} g}{\partial r \partial r \partial x \partial z}+\frac{\partial y \partial z \partial^{2} g}{\partial r \partial r \partial y \partial z}\right),
\end{aligned}
$$

etc.

Then, the first derivatives of the interpolation error of $g$ can be expressed in terms of the Jacobian matrix of the transformation between the physical coordinate system and the normalized coordinate system in the master element, and of the higher order derivatives of $g$ in the physical coordinate system. Sirce the Jacobian matrix can be computed using the geometry of each finite element $\boldsymbol{Y}_{e}$, the remaining issue to be discussed is a method to estimate the second and third derivatives of an unknown function $g$ using its finite element approximation $g_{h}$, spanned by the shape functions of the HEXA8 element for threedimensional problems. Since $\partial g_{h} / \dot{\partial} x, \partial^{2} g_{h} / \partial x \partial y$ and others are computed, for example, at the $2 \times 2 \times 2$ Gauss integration points, their corresponding values at a nodal point can be approximately computed by applying an averaging meihod using their values at the Gauss integration points which surround the node. These avaraged values are then considered to be an estimate of the true derivatives of $g$ at the nodal points. Consequently, assuming that these estimates at the nodal points are interpolated by the same shape functions as those of the HEXA8 element, their derivatives $\partial / \partial x\left(\partial g_{h} / \partial x\right), \partial / \partial x\left(\partial^{2} g_{h} / \partial x \partial y\right)$, etc., are available and can be computed at the $2 \times 2 \times 2$ Gauss integration points. This means that the error $E_{e}^{i j}=a_{Y_{e}}\left(\chi_{I}^{i j}-{ }^{1} \chi^{i j}, \chi_{I}^{i j}-{ }^{1} \chi^{i j}\right)^{1 / 2}$ can be computed. 
Another way to define error measures is based on the sensitivity of the functional

$$
F^{i j}\left(\chi^{H^{i j}}, y_{\alpha}\right)=\frac{1}{2} a_{Y}\left(\chi^{H^{i j}}, \chi^{H^{i j}}\right)-a_{Y}\left(\chi^{H^{i j}}, P^{i j}\right),
$$

whose first variation in $X^{H^{i j}}$ leads to the discrete problem (3.15), that defines the finite element approximation of the equation to determine the characteristic deformations in the base cell, where $y_{\alpha}$ are the coordinates of all the nodes of the finite element model of the base cell $\boldsymbol{Y}$. If the optimum location of the nodes $y_{\alpha}$ is defined so that the functional $F^{i j}$ reaches the minimum, the sensitivity of $F^{i j}$ with respect to the location $y_{\alpha}$ must be zero at the optimum:

$$
\frac{\mathrm{D} F^{i j}}{\mathrm{D} y_{\alpha}}=\frac{\partial F^{i j}}{\partial y_{\alpha}}+\frac{\partial F^{i j}}{\partial x^{H^{i j}}} \frac{\partial X^{H^{i j}}}{\partial y_{\alpha}}=\frac{\partial F^{i j}}{\partial y_{\alpha}}=0
$$

Since $D F^{i j} / D y_{\alpha}$ is computed at every node, its $L^{2}$ norm can be computed at every finite element after interpolating these nodal values by using the shape functions of HEXA8, that is, an error measure $E_{e}^{i j}$ is computed based on the sensitivity of the functional $F^{i j}$. In this case, it is not necessary to estimate the higher order derivatives of $g$ using its finite element approximation $g_{h}$. Only the derivatives of the element stiffness matrix with respect to the nodal coordinates $y_{\alpha}$ is required to define the error measure based on the sensitivity.

EXAMPLE 5.1 (Continuation of Example 3.1). Consider the problem for convergence study in Example 3.1. In this case, non-uniform refinement is considered instead of the uniform refinement based on the adaptive method above described. Several mesh adaptations are

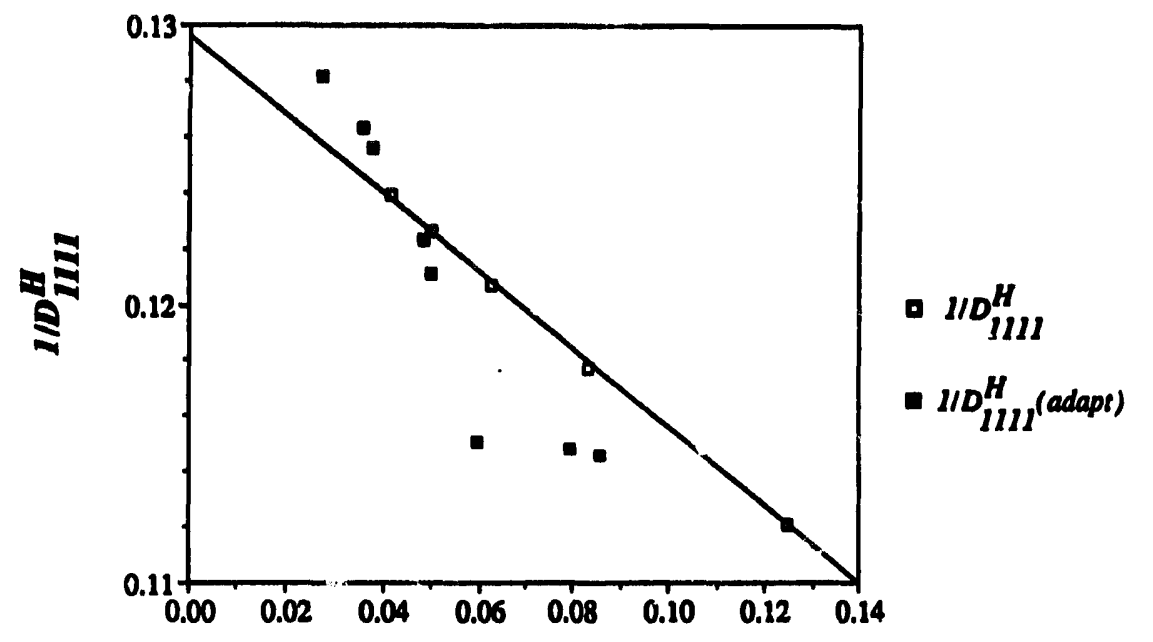

$H=1 / S q r$ (Number of Elements)

$$
1 / D_{111}^{H}=0.102958-0.14055 \mathrm{H}
$$

Fig. 5.1. Convergence of the adaptive method $\left(D_{111}^{H}\right)$. 


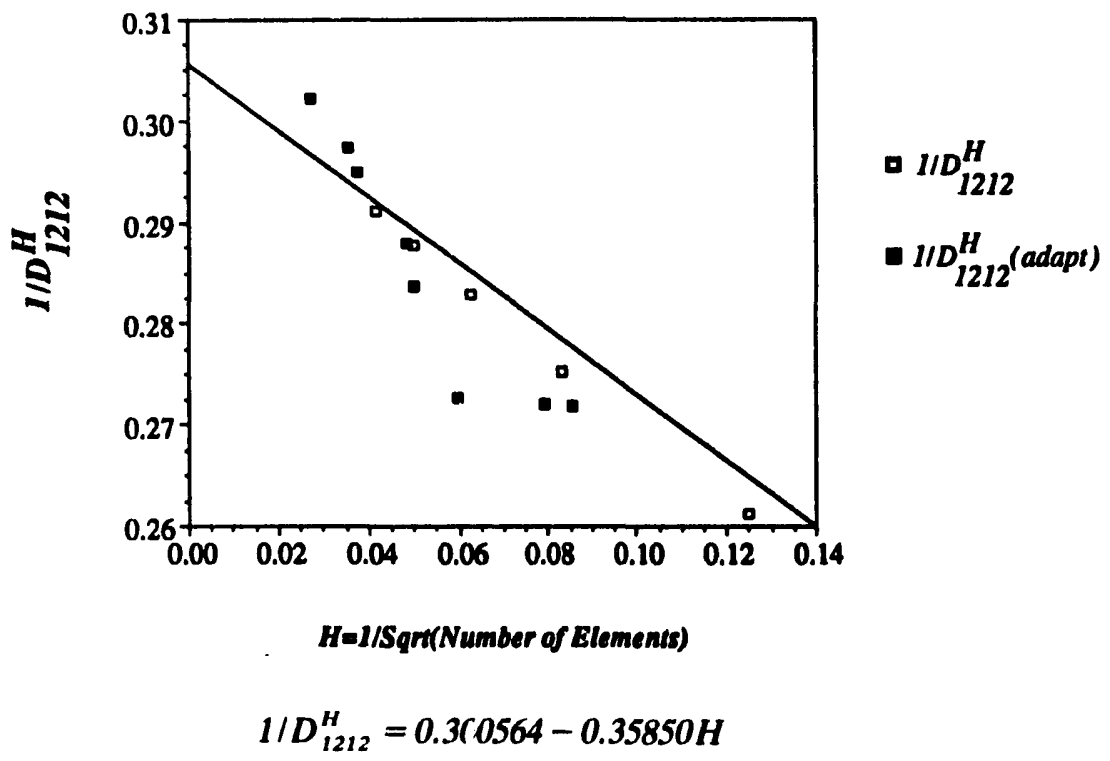

Fig. 5.2. Convergence of the adaptive method $\left(D_{1212}^{H}\right)$.

performed and the corresponding results of the homogenized constants are shown in Fig. 5.4. Some of the mesh adaptations can be found. As one can realize, the adaptive method does not provide better results than the uniform refinement in the range of coarse meshes. However, after a certain number of non-uniform refinements, the adaptive method provides a better finite element result than that obtained with uniform refinements.

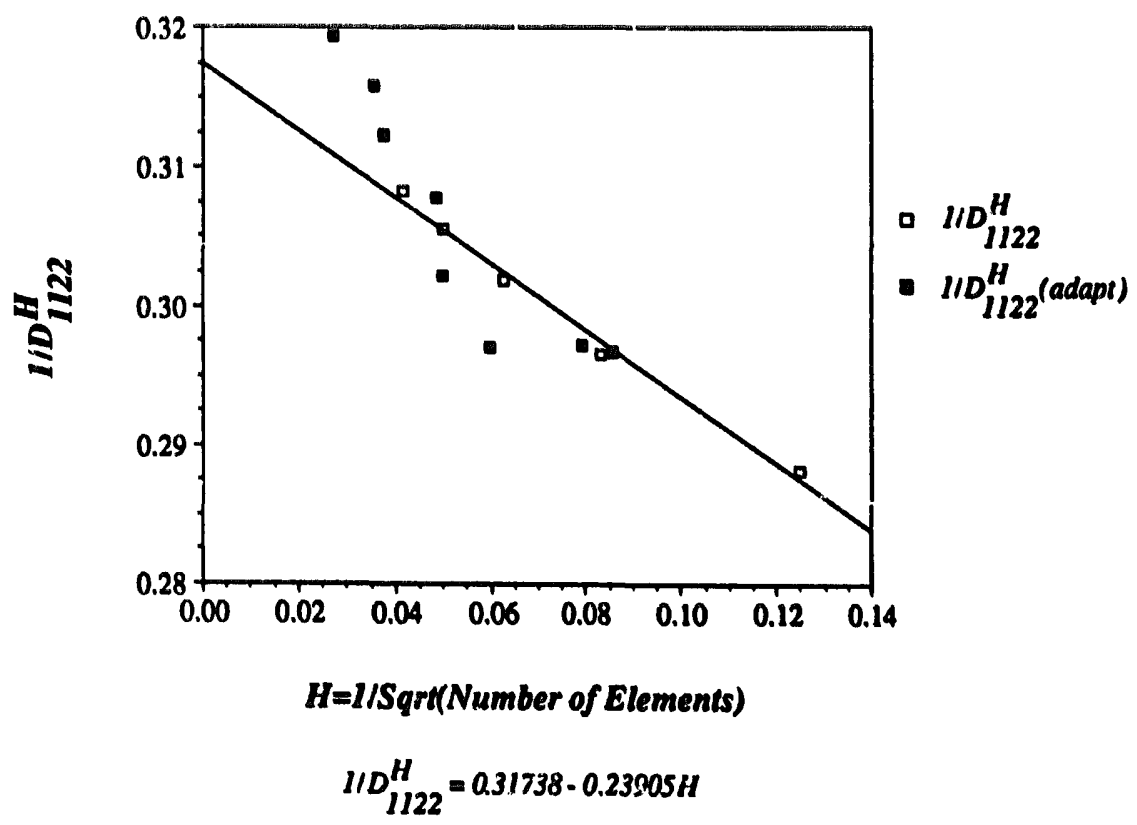

Fig. 5.3. Convergence of the adaptive method $\left(D_{1122}^{H}\right)$. 


\begin{tabular}{|l|l|l|l|l|l|l|l|}
\hline & &, &, &, &, & & \\
\hline & &, &, &, &, & & \\
\hline, &, & & & & &, &, \\
\hline, &, & & & & &, &, \\
\hline, &, & & & & &, &, \\
\hline & &, &, &, &, & & \\
\hline
\end{tabular}

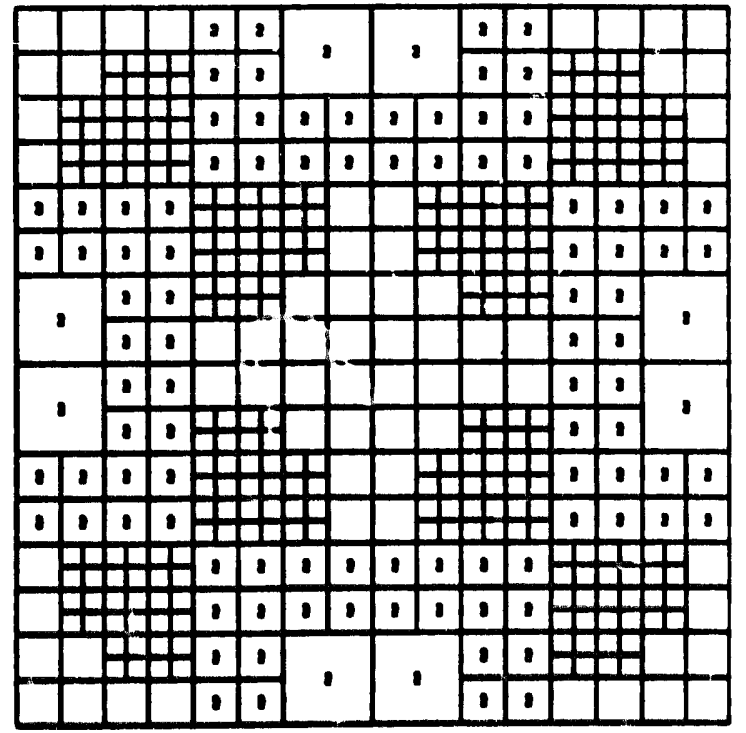

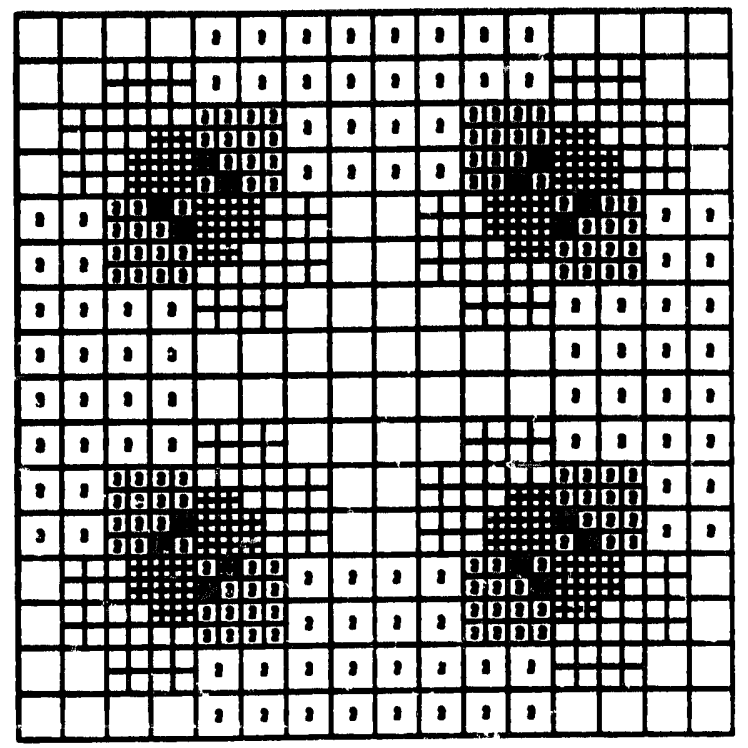

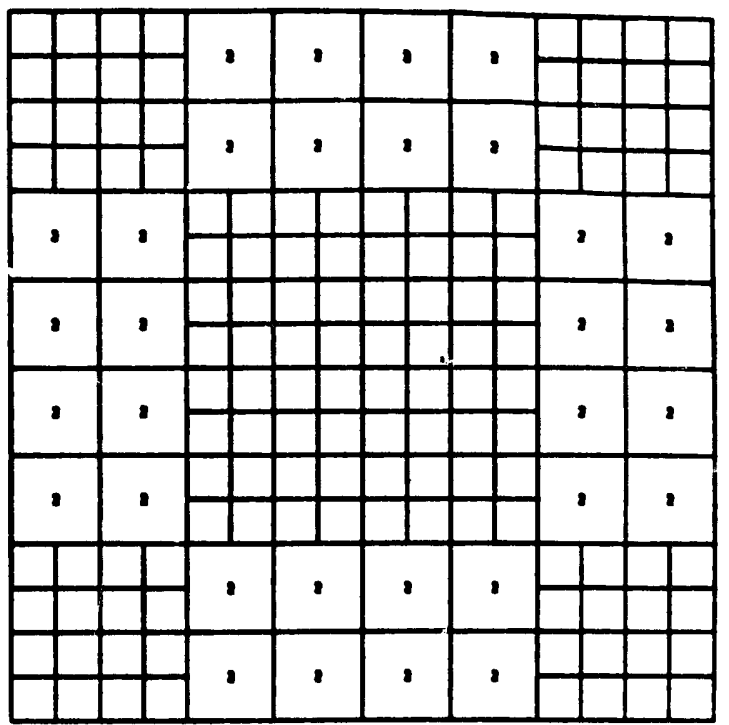

\begin{tabular}{|c|c|c|c|c|c|c|c|c|c|c|c|c|c|c|}
\hline & & & & & 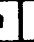 & 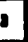 & 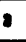 & 1 & 1 & 1 & 1 & & & \\
\hline & & & & & 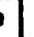 & : & $\bullet$ & : & . & 2 & : & + & & \\
\hline & & & & & & 2 & 2 & 2 & 2 & & & & & \\
\hline & & & & & & 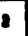 & 2 & $:$ & 8 & & & & I & \\
\hline 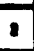 & & & & & & & & & & & & 100 & 2 & 2 \\
\hline 1 & 2 & & 品: & & & & & & & & & 90 & 2 & 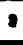 \\
\hline 1 & 2 & 1 & 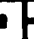 & & & & & & & & & 2 & 2 & 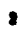 \\
\hline 2 & 1 & 1 & 1 & & & & & & & & & 1 & 1 & 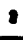 \\
\hline 1 & 1 & 2 & 8 & & & & & & & & & 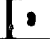 & 1 & $?$ \\
\hline 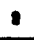 & 1 & 8 & & & & & & & & & & 1 & 1 & ' \\
\hline • & - & an & & & & & & & & & & 100 & 1 & ' \\
\hline 2 & 1 & & & & & & & & 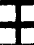 & & $A:$ & jug & : & ' \\
\hline & & & & & & 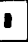 & 1 & 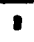 & 1 & & & & & \\
\hline & & & & & 10 & 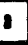 & 1 & 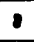 & 1 & III & & & & \\
\hline & & & & & 1 & 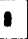 & - & - & : & • & 1 & & & \\
\hline & & & & 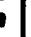 &. & 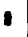 & - & 1 & 1 & • & . & & & \\
\hline
\end{tabular}

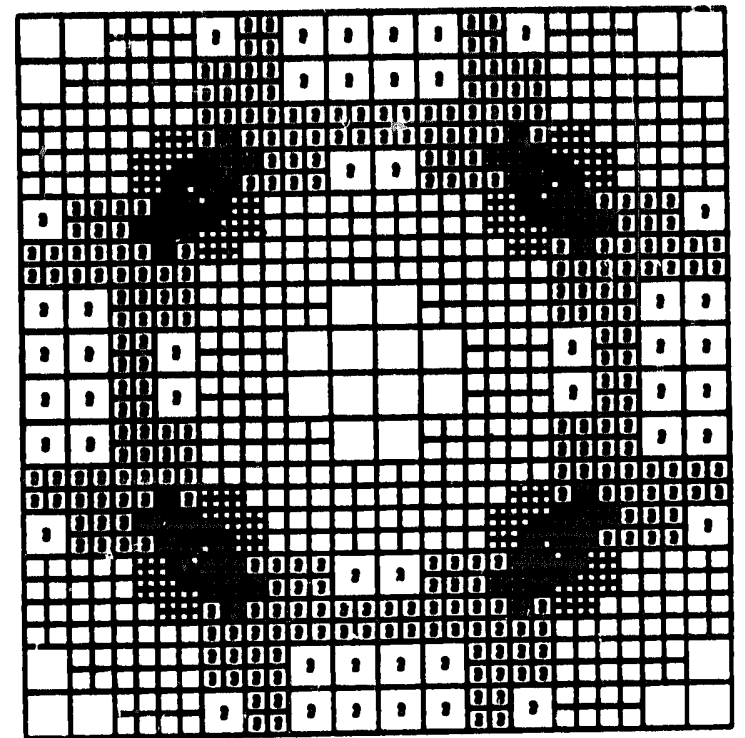

Fig. 5.4. Adapted finite element models (tw)-dimensional problem). 


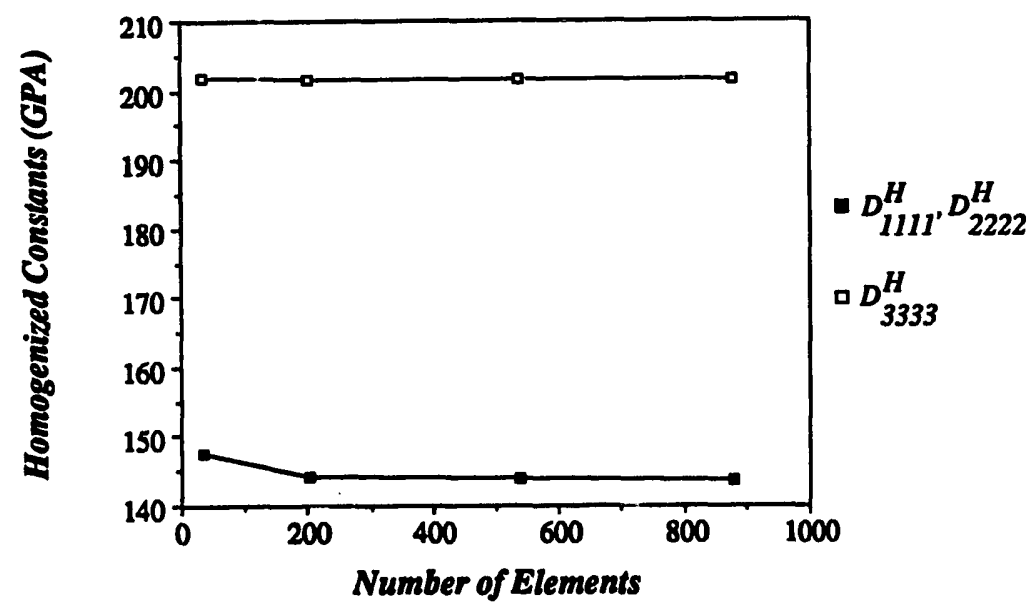

Fig. 5.5. Convergence of the homogenized constants $\left(D_{1111}^{H}, D_{2222}^{H}, D_{3333}^{H}\right)$.

As one can see from Fig. 5.3, for the last adapted finite element mesh, the value of the homogenized coefficients $D_{1122}$ is bigger than the one 'predicted' by the uniform mesh refinements through extrapolation. First this value is only predicted, second, as was noted in (3.22), the finite element solution for $D_{i j k l}^{H}$ is always stiffer than the real one whenever $i j=k l$. However, when this is not the case, like for $D_{1122}$, nothing can be said a priori.

EXAMPLE 5.2 (Continuation of Example 4.2). Consider the three-dimensional adaptive method applied to the base cell problem defined in Example 4.2 to determine the homogenized elastic constants. As shown in Figs. 5.4, 5.5 and 5.6, the adaptive method is effective in the range of coarse meshes. Once the number of finite elements reaches to a certain limit, improvement of the accuracy of the finite element approximation becomes very small. Uniform refinements were not performed for this example because the size of the finite element mesh becomes very large after two refinements.

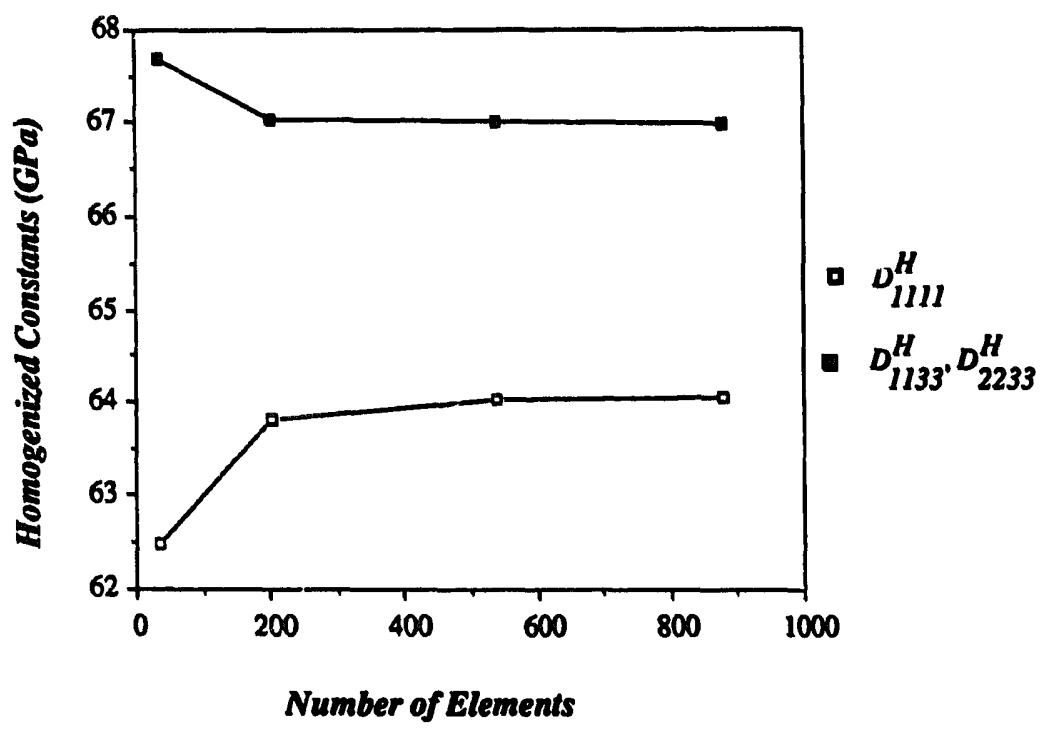

Fig. 5.6. Convergence of the homogenized constants $\left(D_{1111}^{H}, D_{1133}^{H}, D_{2233}^{H}\right)$. 


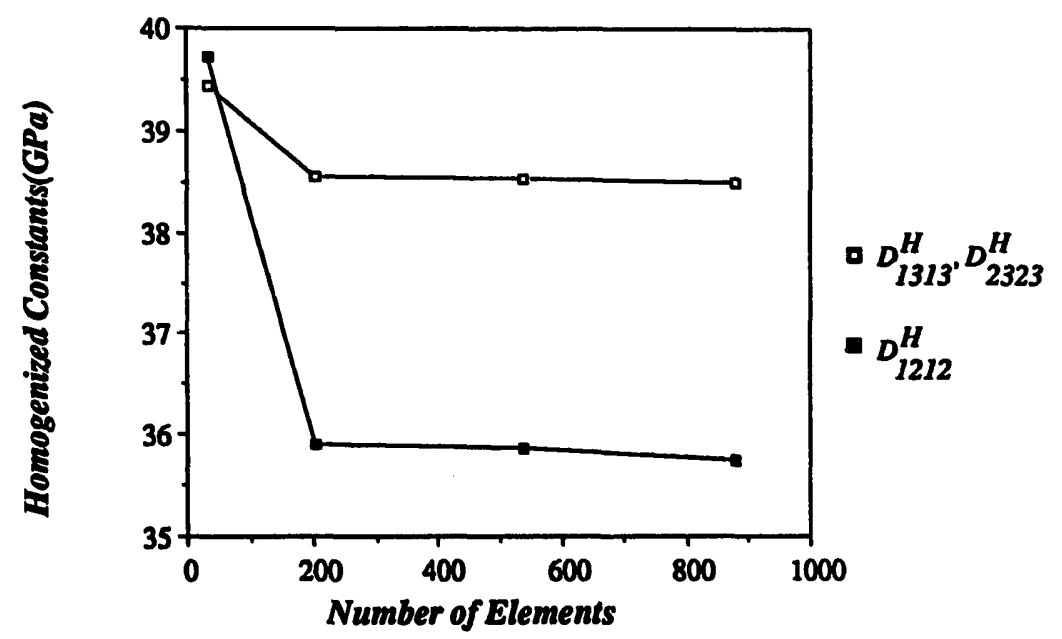

Fig. 5.7. Convergence of the homogenized constants $\left(D_{2323}^{H}, D_{1313}^{H}, D_{1212}^{H}\right)$.
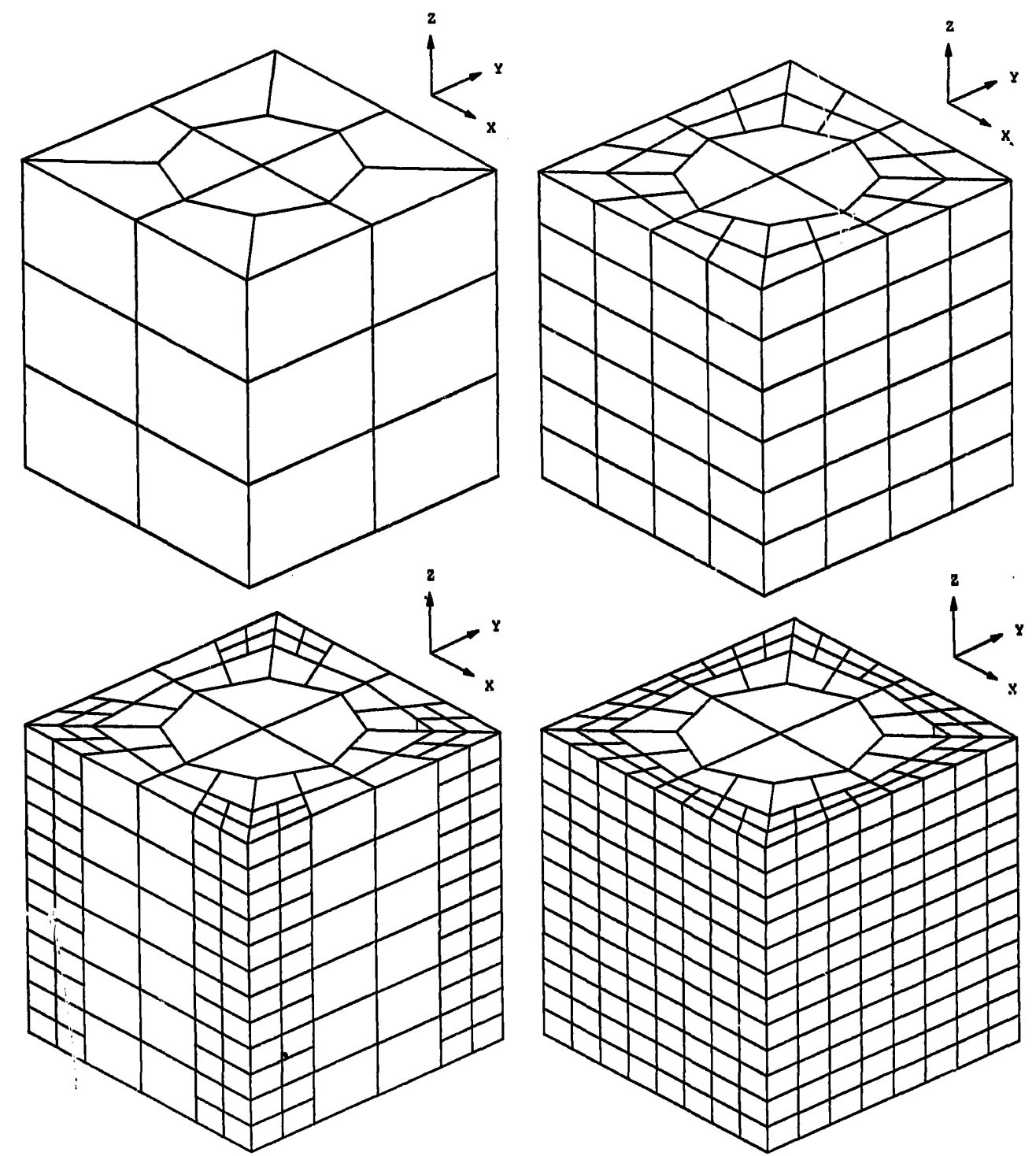

Fig. 5.8. Adapted finite element models (three-dimensional problem). 


\section{Final remarks}

In this paper the homogenization method was introduced to model the mechanical behavior of linear elastic composite materials. This method assumes that the composite material has a periodic microstructure, and it enables the computations of equivalent (homogenized) materiIl mechanical properties characterizing the overall behavior of the composite, as well as the local one, i.e., it enables the computation of local stress and strain distribution within the inicrostructure of the composite.

The numerical implementation of this method is introduced via a finite element technique, and a priori error estimations were derived for the solutions of the problems involving the composite microstructure and the global structure. A convergence study was done for a two-dimensional case providing a result compatible with the previous a priori error estimations.

Then, the homogenization method is used to introduce the idea of a material preprocessor (PREMAT) for the computation of the homogenized properties of composites materials, and a material postprocessor (POSTMAT) for the computation of the stress and strain distribution within the composite microstructure. The implementation procedure is explained and several application examples are presented. These examples show the usefulness of PREMAT and POSTMAT capabilities. They enable the designer to model the mechanical response of composite materials, when there is no experimental data available, and they provide a first approximation of the stress distribution on the composite microstructure. Also, for the composite designer, it provides a tool to study the influence of the geometric parameters of the microstructure in composite global response, as well as to locate the possible critical points of the microstructure where high stress concentration may occur. Also, it may be useful to try to understand what can eventually trigger the failure of the composite.

Finally, an adaptive finite element method is introduced in order to improve the accuracy of the numerical results. An error measure is suggested for the homogenized material constants, based on the a priori error estimations and the numerical implementation presented. The adaptive method is then applied to $2 \mathrm{D}$ and $3 \mathrm{D}$ examples. The $2 \mathrm{D}$ case was compared with uniform mesh refinements of the previously made convergence study. After some mesh adaptations the method presented better results than the corresponding uniform refinements. However, one might note that, if the regularity of the solution of the involved problems is known or can be, somehow, 'guessed', the a priori error estimations provide a convergence rate, and one may be able, after performing some uniform mesh refinements, to extrapolate the 'exact' values of the homogenized constants.

\section{Acknowledgment}

The authors are supported by NASA Lewis Research Center, NAG 3-661 and Office of Naval Research, ONR N-00014-88-K-0637, while this work was performed. The programs PREMAT and POSTMAT are developed by the support from RTB Corporation and QUINT Co. The authors express their sincere appreciation to these supports. 


\section{References}

[1] Z. Hashin, Theory of composite materials, in: F.W. Wend, H. Liebowitz and N. Perrone, eds., Mechanical of Composite Materials (Pergamon, Oxford, 1970).

[2] J.L. Lions, Some Methods in the Mathematical Analyses of Systems and their Control (Science Press, Beijing, China, 1981) and (Gordon and Breach, New York).

[3] A. Benssousan, J.L. Lions and G. Papanicoulau, Asymptotic Analysis for Periodic Structures (NorthHolland, Amsterdam, 1978).

[4] E. Sanchez-Palencia, Non Homogeneous Media and Vibration Theory, Lecture Notes in Physics, No. 127 (Springer, Berlin, 1980).

[5] G. Duvaut, Analyse functionelle et mécanique des milieux continues. Applications à l'etude de matérieux composites elastiques à structure périodiques. Homogénéisation, in: W.T. Koiter, ed., Theoretical and Applied Mechanics (North-Holland, Amsterdam, 1976) 119-132.

[6] G. Duvaut, Homogeneization et materiaux composite, in: P.G. Ciarlet and M. Rouseau, eds., Trends and Applications of Pure Mathematics to Mechanics, Lecture Notes in Physics No. 195 (Springer, Berlin, 1984).

[7] F. Lene, Thése de Doctorat d'Etat, Université Pierre et Marie Curie, Paris VI, 1984.

[8] O.A. Oleinik, On homogenization problems, in: P.G. Ciarlet and M. Rouseau, eds., Trends and Applications of Pure Mathematics to Mechanics (Springer, Berlin, 1984).

[9] F. Murat and L. Tartar, Calculs des variations et homogênéization, in: Les Methodes de l'Homogenéization: Theory et Applications en Physique (Coll. de la Dir. de Etudes et Recherches d'Electricité de France, Eyrolles, 1985) 319.

[10] R. Kohn, Recent progresses in the mathematical modeling of composite materials, Courrant Institute, New York.

[11] J.R. Willis, Bounds and selfconsistent estimates for overall properties of anisotropic composites, J. Mech. Phys. Solids 25 (3) (1977) 389-393.

[12] E. Kroner, Effective moduli of random elastic media-Unified calculation of bounds and self consistent values, Mech. Res. Comm. 4 (6) (1977) 389-393.

[13] Begis, S. Dinari, G. Duvaut, A. Hassim and F. Pistre, MODULEF and composite materials, INRIA, Rocquencourt, Le Chesnay, Preprint.

[14] J. Necas and I. Hlavacek, Mathematical Theory of Elastic and Elastico-Plastic Bodies: An Introduction (Elsevier, Amsterdam, 1981).

[15] D. Cioranescu, Thesis, Paris, 1978.

[16] D. Cioranescu and J.S.J. Paulin, Homogenization in open sets with holes, J. Math. Anal. Appl. 71 (1979) 590-607.

[17] J.T. Oden and J.N. Reddy, An Introduction to the Mathematical Theory of Finite Elements (Wiley, New York, 1976).

[18] P.G. Ciarlet, The Finite Element Method for Elliptic Problems (North-Holland, Amsterdam, 1978).

[19] I. Babuška and A.K. Aziz, Survey lectures on the mathematical foundations of the finite element method, in: A.K. Aziz, ed., The Mathematical Foundations of the Finite Element Method with Applications to Partial Differential Equations (Academic Press, New York, 1972).

[20] B.C. Koh and N. Kikuchi, New improved hourglass control for bilinear and trilinear elements in anisotropic linear elasticity, Comput. Methods Appl. Mech. Engrg. 65 (1) (1987) 1-46.

[21] C.T. Lynch ed., CRC_Handbook of Materials Science Vol. II (CRC Press, Cleveland, 1975) 392.

[22] E.R. Arantes E Oliveira, Optimization of the finite element solution, in: Proc. Third Conference of Matrix Methods in Structural Mechanics (Wright-Patterson Air Force Base, Dayton, OH, 1971).

[23] I. Babuška, The adaptive methods and error estimation for elliptic problems of structural mechanics, Proc. ARO Workshop on Adaptive Methods for Partial Differential Equations, Univ. of Maryland, College Park, 14-16 February 1983.

[24] I. Babuška, and W.C. Rheinboldt, Reliable error estimation and mesh adaptation for the finite element method, in: J.T. Oden, ed., Computational Methods in Nonlinear Mechanics (North-Holland, Amsterdam, 1980) 67-109. 
[25] I. Babuška and W.C. Rheinboldt, Error estimates for adaptive finite element computations, Internat. J. Numer. Methods Engrg. 15 (1980) 736-754.

[26] I. Babuška and M.R. Dorr, Error estimates for the combined $h$ and $p$ version of the finite element method, Numer. Math. 25 (1981) 257-277.

[27] A.R. Diaz, N. Kikuchi and J.E. Taylor, Optimal designi formulations for finite element grid adaptation, in: V. Komkov, ed., Sensitivity of Functionals with Applications to Engineering Science, Lecture Notes in Mathematics 1086 (Springer, Berlin, 1984) 56-76.

[28] A.R. Diaz, N. Kikuchi and J.E. Taylor, A method of grid optimization for finite element methods, Comput. Methods Appl. Mech. Engrg. 41 (1) (1983) 29-45.

[29] N. Kikuchi, Adaptive grid design methods for finite element analysis, Comput. Methods Appl. Mech. Engrg. 55 (1986) 129-160. 